\title{
Republic of Moldova: Second Review Under the Three-Year Arrangement Under the Poverty Reduction and Growth Facility and Request for Waiver of Nonobservance of Performance Criterion-Staff Report; Staff Statement; Staff Supplement; Press Release on the Executive Board Discussion; and Statement by the Executive Director for the Republic of Moldova
}

In the context of the second review under the three-year arrangement under the Poverty Reduction and Growth Facility and request for a waiver of nonobservance of a performance criterion, the following documents have been released and are included in this package:

- $\quad$ The staff report for the Second Review Under the Three-Year Arrangement Under the Poverty Reduction and Growth Facility and Request for Waiver of Nonobservance of Performance Criterion, prepared by a staff team of the IMF, following discussions that ended on May 8, 2007, with the officials of the Republic of Moldova on economic developments and policies. Based on information available at the time of these discussions, the staff report was completed on June 25, 2007. The views expressed in the staff report are those of the staff team and do not necessarily reflect the views of the Executive Board of the IMF.

- $\quad$ A supplement on the joint World Bank/IMF debt sustainability analysis.

- A staff statement of July 13, 20007 updating information on recent developments.

- $\quad$ A Press Release summarizing the views of the Executive Board as expressed during its discussion of the staff report that completed the review and request.

- A statement by the Executive Director for the Republic of Moldova.

The documents listed below have been or will be separately released.

Letter of Intent sent to the IMF by the authorities of the Republic of Moldova*

Memorandum of Economic and Financial Policies by the authorities of the Republic of Moldova*

Technical Memorandum of Understanding*

*Also included in Staff Report

The policy of publication of staff reports and other documents allows for the deletion of market-sensitive information.

To assist the IMF in evaluating the publication policy, reader comments are invited and may be sent by e-mail to publicationpolicy@imf.org.

Copies of this report are available to the public from

International Monetary Fund • Publication Services

$70019^{\text {th }}$ Street, N.W. • Washington, D.C. 20431

Telephone: (202) 623-7430 • Telefax: (202) 623-7201

E-mail: publications@imf.org • Internet: http://www.imf.org

Price: $\$ 18.00$ a copy

International Monetary Fund

Washington, D.C. 

INTERNATIONAL MONETARY FUND

REPUBLIC OF MOLDOVA

\title{
Second Review Under the Three-Year Arrangement Under the Poverty Reduction and Growth Facility and Request for Waiver of Nonobservance of Performance Criterion
}

\author{
Prepared by the European Department \\ (In consultation with other departments)
}

Approved by Juha Kähkönen and Anthony R. Boote

June 25, 2007

\section{Executive Summary}

The economic impact of the external shocks faced in 2006 seems less severe than expected at the time of the first review. Although wine exports to Russia have not resumed, other markets are being found, and a better-than-expected deal for natural gas deliveries was struck. Thus, growth has recovered to around 5 percent, while reserves are strengthening and inflation is coming down.

Program performance has been generally satisfactory. The quantitative performance criteria were observed, as was most of the structural conditionality. One structural performance criterion was missed at end-December: the increase in tariffs for district heat and water was briefly delayed in Chişinău. A related quantitative benchmark — on nonaccumulation of domestic expenditure arrearswas breached when the Chişinău city council neglected to pay for energy subsidies it decided to grant to households.

The attached MEFP underscores the authorities' commitment to growth-enhancing reforms, while coming against a background of increasingly fractious politics. The electoral cycle has stimulated public sector wage increases and a tax/capital amnesty, but the authorities are taking measures to maintain a prudent fiscal stance. The next review will focus on the composition of expenditures to ensure that fiscal policy remains pro-growth and pro-poor.

The ceiling on nonconcessional borrowing would be increased, mainly to accommodate a roads project financed by the EBRD and the EIB. The authorities are determined to bolster debt sustainability by securing sufficient grants so that the second phase of the project has a grant element of at least 35 percent.

Staff supports completion of the review and granting a waiver for nonobservance of the structural performance criterion. The authorities' commitment to implement supplementary measures provides assurance that the program's objectives remain attainable. 


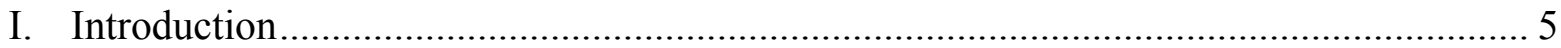

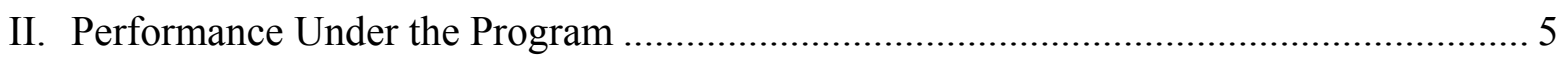

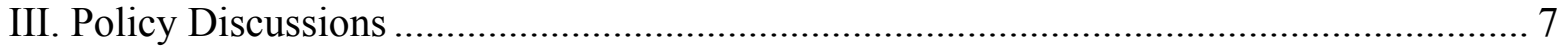

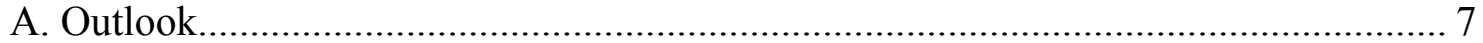

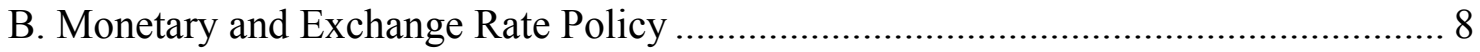

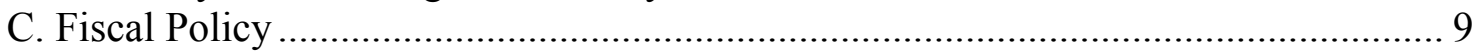

D. Financial Sector Stability and Reforms …………............................................ 10

E. Structural Reforms..................................................................................... 11

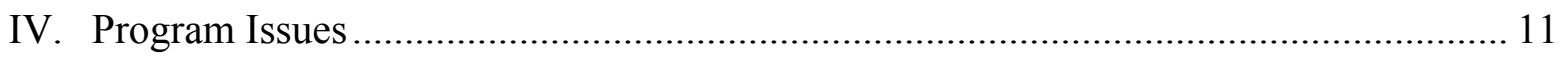

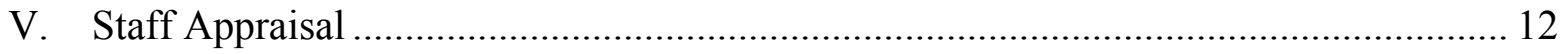

Text Tables

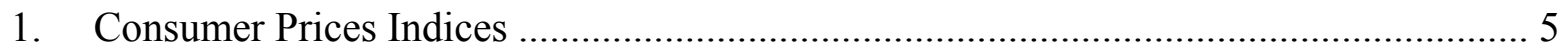

2. General Government Fiscal Balance, 2003-07.......................................................... 6

3. Medium-Term Outlook: Program and New Baseline Scenarios, 2005-09 ..................... 7

Figures

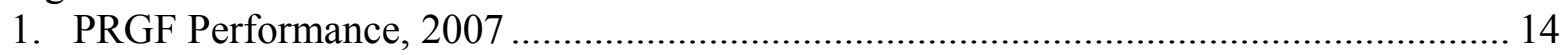

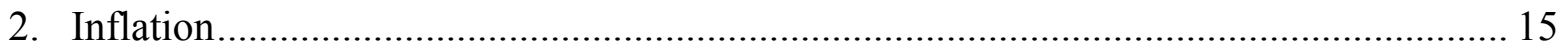

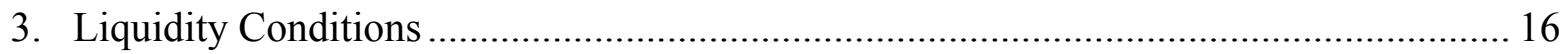

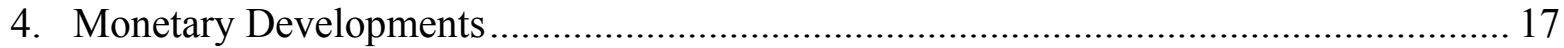

Tables

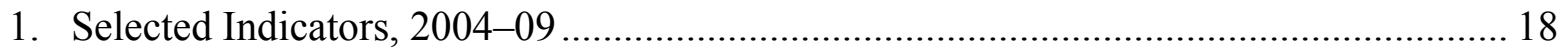

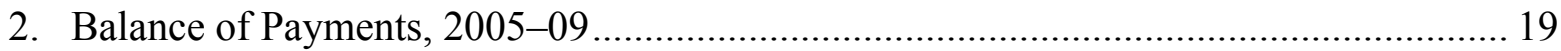

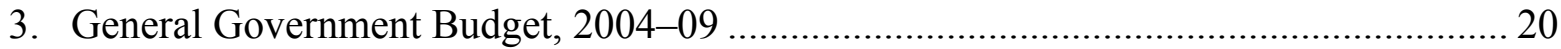

3a. General Government Budget, 2006-07 ……………................................................ 22

4. Accounts of the National Bank of Moldova and Monetary Survey, 2005-07................. 23

5. Financial Sector Indicators, 2000-06 ……………................................................... 24

6. Localized Millennium Development Goals (EGPRSP) ................................................. 25

7. External Financing Requirements and Sources, 2004-09 .......................................... 26

8. Quantitative Performance Criteria and Indicative Targets, December 31, 2006-

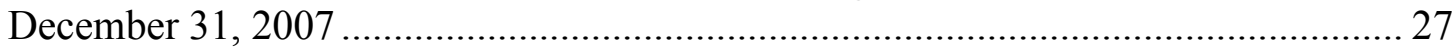

9. Status of Structural Performance Criteria and Benchmarks ……………………........... 28

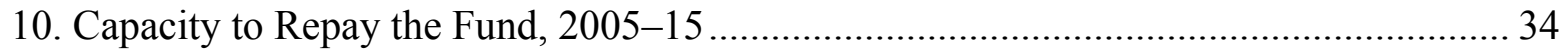

11. Reviews and Disbursements Under the Three-Year PRGF Arrangement........................ 35 
Attachments

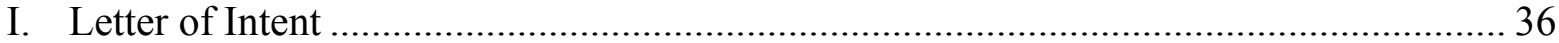

II. Updated Memorandum of Economic and Financial Policies for 2007 .......................... 38

III Technical Memorandum of Understanding ........................................................... 49 


\section{List of Acronyms}

\begin{tabular}{ll} 
AML & Anti-Money Laundering \\
ANRE & National Agency for Energy Regulation \\
BEM & Banca de Economii \\
BoP & Balance of payments \\
CCECC & Center for Combating Economic Crime and Corruption \\
CEB & Council of Europe Development Bank \\
CG & Consultative group of donors \\
CIT & Corporate income tax \\
DfID & UK Department for International Development \\
DSA & Debt sustainability analysis \\
EBRD & European Bank for Reconstruction and Development \\
EGPRSP & Economic Growth and Poverty Reduction Strategy Paper \\
EIB & European Investment Bank \\
ENPI & EU European Neighborhood Policy Instrument \\
EUR & IMF European Department \\
FAD & IMF Fiscal Affairs Department \\
FSAP & Financial Sector Assessment Program \\
IFC & International Finance Corporation \\
LOI & Letter of Intent \\
MFA & EU Macro-Financial Assistance \\
MEFP & Memorandum on Economic and Financial Policies \\
MTEF & Medium Term Expenditure Framework \\
OMOs & Open market operations \\
NBFI & Nonbank financial institution \\
NBM & National Bank of Moldova \\
NCFM & National Commission on the Financial Market \\
PER & Public expenditure review \\
PIT & Personal income tax \\
PRSC & World Bank Poverty Reduction Support Credit \\
PSIA & Poverty and Social Impact Analysis \\
SF & Social Fund \\
SIDA & Swedish International Development Agency \\
STI & State Tax Inspectorate \\
tcm & thousand cubic meters \\
\hline FA &
\end{tabular}




\section{INTRODUCTION}

1. While the external environment is improving, the domestic political cycle is complicating economic decision making. The authorities remain committed to the PRGFsupported program, particularly in light of December's CG meeting. However, recent local government elections (where the ruling Communist Party did poorly) and parliamentary elections in early 2009 have begun to distract policymakers. There is also much talk - though nothing concrete - of a possible settlement of the Transnistria dispute.

\section{Performance Under the Program}

\section{The external shocks faced by Moldova now look to be less severe than expected} at the time of the first review.

Natural gas will be imported at

$\$ 170 /$ tcm in 2007 (instead of the near-

European price of $\$ 220 / \mathrm{tcm}$ expected

in December), rising gradually to

European prices over five years.

Although Russia has formally lifted its ban on imports of Moldovan wine, the modalities for resuming shipments have not been settled. Nevertheless, wine exports in 2006 exceeded expectations, as alternative markets are

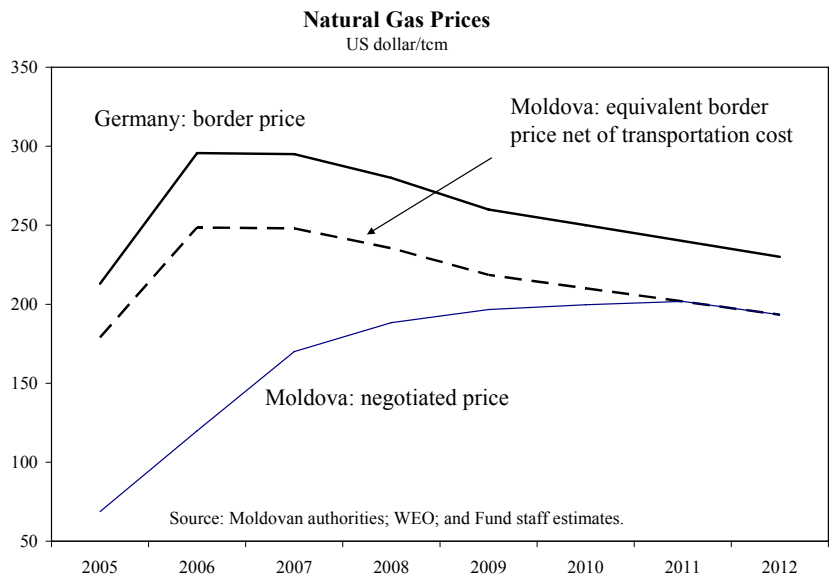
gradually being tapped.

\section{Growth has been healthy in light of the better-than-expected external} environment. At 4 percent, real GDP growth in 2006 exceeded projections, and industrial production has started to recover. Growth continues to be fueled by remittances, which were 33 percent of GDP in 2006.

\section{While high by regional standards, inflation is abating, as the impact of last} year's energy price hikes ebbs. Twelvemonth headline inflation fell from $14 \frac{1}{2}$ percent in December to $10^{1 / 2}$ percent in May. Although underlying (nonfood, nonenergy) inflation remained high at 13.9 percent, annualized inflation fell below 10 percent, suggesting the authorities' endyear goal of single-digit inflation is attainable.

\begin{tabular}{lcrr} 
& $\begin{array}{c}\text { Consumer Price Indicies } \\
\text { (twelve months change) }\end{array}$ & & \\
\hline & Dec & Dec & Mar \\
& 2005 & 2006 & 2007 \\
\hline Moldova & 10.0 & 14.1 & 11.2 \\
Romania & 8.6 & 4.9 & 3.7 \\
Macedonia, FYR & 1.3 & 3.1 & 1.5 \\
Ukraine & 10.3 & 11.6 & 10.1 \\
Russia & 10.9 & 9.0 & 7.4 \\
\hline Sources: Country authorities; and IFS. & &
\end{tabular}




\section{Despite unexpectedly robust foreign exchange inflows, monetary policy has} contributed to disinflation. The

NBM has responded by stepping up sterilization and accelerating

reserve accumulation. By late May, gross reserves were well above the end-2007 target established at the First Review. Real interest rates have been positive since December, and the leu has appreciated against the dollar in nominal terms by about 4 percent since end-December. The endMarch monetary targets were met—-for NDA and NIR by a wide margin.

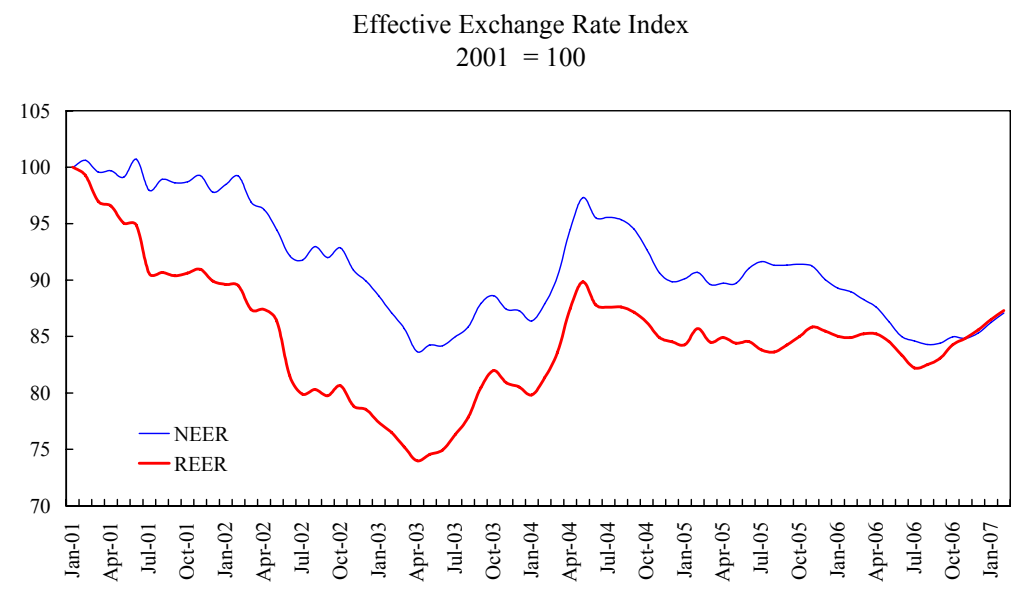

Source: IMF staff calculations

6. Moldova continues to overperform against its fiscal deficit targets. Driven mainly by revenue overperformance - especially VAT and excises on imports - the 2006 and Q1 2007 fiscal deficit targets were observed with a margin. The 2006 fiscal outturn was a surplus of 0.3 percent of GDP, excluding the 0.6 percent of GDP recapitalization of the NBM.

General Government Fiscal Balance, 2003-07

(in percent of annual GDP)

\begin{tabular}{lccccc}
\hline & 2003 & 2004 & 2005 & 2006 & Q1 2007 \\
\hline Outturn & 0.2 & 0.8 & 1.7 & 0.3 & 0.8 \\
Initial budget & -0.7 & -0.9 & -0.9 & -0.5 & -0.3 \\
\hline
\end{tabular}

Sources: Moldovan authorities and Fund staff estimates.

\section{Implementation of structural conditionality was broadly satisfactory, though a}

few measures were delayed. Legislation to strengthen the NBM's capital structure was implemented well ahead of schedule. Tariffs for district heat were increased on January 25, rather than by end-December, though the move had retroactive effect. Water tariffs were increased in February. (Because of the delay, the authorities are seeking a waiver for missing this PC.) While tariffs were raised to cost recovery, the Chişinău municipality decided to subsidize households temporarily for the difference between the previous heating tariff and the cost recovery level. (The weighted average effective tariff exceeds the 55 percent threshold in the program even so.) However, by delaying transfers to the public utility to cover the cost of the subsidy, the municipality caused a breach of the indicative ceiling on domestic expenditure arrears of the general government ( 0.3 percent of GDP $).{ }^{1}$ Submission

\footnotetext{
${ }^{1}$ Chişinău began to clear arrears in April.
} 
of the draft civil service law was delayed to allow time for donor comments, and because of political sensitivity in advance of June elections. The end-March benchmark on passage of the law establishing the NCFM was delayed as vested interests fought to prevent stronger supervision of the insurance sector.

\section{Policy Discussions}

\section{Abatement of the external shocks of 2006 allowed the discussions to refocus on} the core objectives of the PRGF-supported program. These are to promote faster growth and poverty reduction by: (i) ensuring macroeconomic stability, (ii) enhancing financial sector stability and development, and (iii) reducing the footprint of the state in the economy, including through reforms aimed at improving the business environment.

\section{A. Outlook}

\section{At 5 percent, growth is expected to remain strong, fed in the short run by} remittances. Over the medium term, investment should become the engine of growth, supported by a recovery of the wine sector. Inflation is projected to drop below 10 percent in 2007 , and with time to fall to partner-country levels.

Medium-Term Outlook: Program and New Baseline Scenarios, 2005-09

\begin{tabular}{|c|c|c|c|c|c|c|c|c|}
\hline & \multirow[t]{2}{*}{2005} & \multicolumn{2}{|c|}{2006} & \multicolumn{2}{|c|}{2007} & \multicolumn{2}{|c|}{2008} & \multirow{2}{*}{$\frac{2009}{\text { Proj. 2/ }}$} \\
\hline & & $\begin{array}{c}\text { IMF CR } \\
\text { No. } 07 / 45\end{array}$ & Prel. & $\begin{array}{c}\text { IMF CR } \\
\text { No. } 07 / 45\end{array}$ & Proj. 2/ & $\begin{array}{c}\text { IMF CR } \\
\text { No. } 07 / 45\end{array}$ & Proj. 2/ & \\
\hline \multicolumn{9}{|c|}{ (Percent change) } \\
\hline Real GDP & 7.5 & 3.0 & 4.0 & 3.0 & 5.0 & 5.0 & 5.0 & 5.0 \\
\hline Investment (percent. point contrib. to growth) & 2.0 & -0.4 & 2.3 & 0.0 & 1.4 & 0.8 & 1.5 & 1.5 \\
\hline Inflation (end of period) & 10.0 & 12.0 & 14.1 & 10.0 & 10.0 & 8.0 & 8.0 & 7.0 \\
\hline Real effective exchange rate & -1.3 & 1.8 & 0.0 & $\ldots$ & $\ldots$ & $\ldots$ & $\ldots$ & $\ldots$ \\
\hline Reserve money & 31.8 & -5.6 & -7.0 & 9.7 & 14.3 & $\ldots$ & $\ldots$ & $\ldots$ \\
\hline \multicolumn{9}{|c|}{ (In percent of GDP, unless otherwise noted) } \\
\hline Current account balance & -10.3 & -10.4 & -12.0 & -7.2 & -8.9 & -4.2 & -8.2 & -7.1 \\
\hline Wine exports & 10.8 & 4.8 & 5.6 & 6.1 & 5.7 & 7.1 & 6.4 & 7.2 \\
\hline Energy imports & 14.6 & 19.2 & 16.2 & 22.9 & 16.0 & 22.4 & 15.3 & 14.2 \\
\hline Remittances & 29.1 & 36.5 & 33.3 & 40.2 & 33.7 & 41.0 & 33.2 & 33.4 \\
\hline Gross official reserves, months of imports & 2.2 & 2.3 & 2.5 & 2.4 & 2.9 & 2.7 & 3.3 & 3.9 \\
\hline \multicolumn{9}{|l|}{ General Government } \\
\hline Revenues & 39.5 & 40.3 & 40.5 & 42.4 & 42.0 & 41.0 & 39.7 & 39.5 \\
\hline Expenditures & 37.9 & 40.3 & 40.2 & 42.9 & 42.5 & 41.6 & 40.2 & 40.0 \\
\hline Balance 1/ & 1.7 & 0.0 & 0.3 & -0.5 & -0.5 & -0.5 & -0.5 & -0.5 \\
\hline GDP, billions of lei & 36.8 & 42.0 & 44.1 & 46.6 & 49.7 & 53.2 & 56.0 & 62.9 \\
\hline
\end{tabular}

Sources: Moldovan authorities; and Fund staff projections.

1/ Excludes injection of 0.6 percent of GDP to NBM capital in late 2006.

2/ Projections represent the program scenario. 


\section{B. Monetary and Exchange Rate Policy}

10. Higher foreign exchange inflows will continue to complicate monetary policy, which needs to remain tight to achieve single-digit inflation. In light of the weaker impact of the external shocks, it is proposed that the NIR and NDA targets be tightened to take account of recent and expected overperformance. The indicative reserve money ceiling (the authorities' operational target) would be increased to factor in higher nominal GDP growth in 2006, implying reserve money growth of 14 percent and broad money growth of 18 percent in 2007.

11. The NBM is well placed to manage monetary policy, despite strong inflows:

- $\quad$ The authorities understand that interest rates need to remain relatively high until disinflation is entrenched, and the NBM is prepared to step up sterilization accordingly;

- The costs of sterilization and valuation losses due to appreciation-while highremain manageable, given improvements in the NBM's income position stemming from growing income from foreign reserves, higher interest on the NBM's portfolio of government paper, and the strengthening in the NBM's capital position at the end of 2006;

- $\quad$ The stock of NBM claims on the government is to be securitized, bolstering the Bank's ability to conduct OMOs; and

- $\quad$ Liquidity pressures will ease in late 2007, when the balances of the Social and Health Funds are moved to the Treasury account in the NBM.

12. The program aims to balance the need for higher reserves against the support for disinflation afforded by nominal appreciation.

Offical Foreign Exchange Reserves, 2006

While broadly allowing the market to set the exchange rate, the NBM expects gross reserves to reach almost $\$ 1$ billion (nearly three months of imports) by end2007, somewhat above the level projected in the original program, but still low by regional comparison.

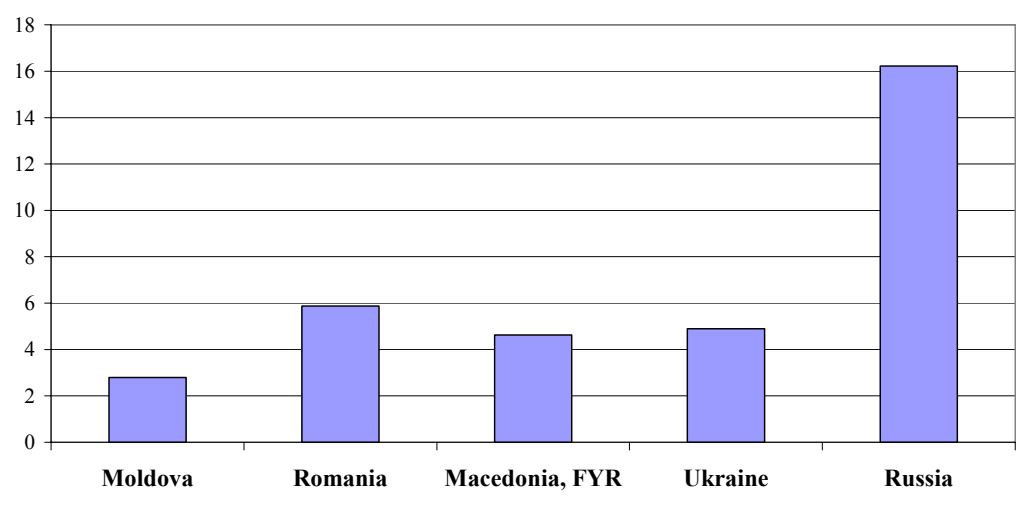




\section{Fiscal Policy}

13. The authorities are resisting election-related pressure to relax the fiscal stance in 2007. They are working on a supplementary budget to allocate $1 \frac{1}{4}$ percent of GDP in excess revenue collected through April. Although additional revenue overperformance is likely during the last 8 months (staff projects a further 1 percent of GDP in unallocated revenue), the authorities will defer another supplementary budget until the revenue estimates are more certain.

14. At the same time, two recent policy developments pose risks for the 2008 budget:

- $\quad$ Parliament has passed an increase in government wages that, being introduced in stages during the year, will add about $1 / 2$ percent of GDP to spending in 2007 - easily covered by the revenue overperformance noted above. In 2008, however, the increase threatens to widen the general government wage bill to 11 percent of GDP in the absence of measures, crowding out investment needed for growth and poverty reduction.

- At the behest of President Voronin, the authorities have adopted a package of measures, which will entail a short-run revenue loss that could also squeeze out propoor, pro-growth spending. The President's initiatives include introducing a zero rate for the CIT (except for dividends and nonbusiness expenses), a broad amnesty of pre2007 arrears, and a liberalization of capital repatriation rules.

\section{To prevent the wage increase from undermining poverty-reduction efforts, the} authorities plan to downsize public sector employment over the medium term. The authorities agree with the findings of the recent World Bank PER that the number of budget sector workers is high by international comparison (particularly in the education sector). Indeed, the recently-approved MTEF for 2008-10 explicitly recognizes that wage increases need to be accompanied by downsizing, and some initial steps have already been taken (redundancies of police and some military). Accordingly, by endSeptember the government will develop a medium-term approach to rationalize public sector employment (with World Bank support). In the meantime - as a temporary measure - an indicative ceiling on the general government wage bill (10 percent of GDPconsistent with the MTEF) has been added to the program.

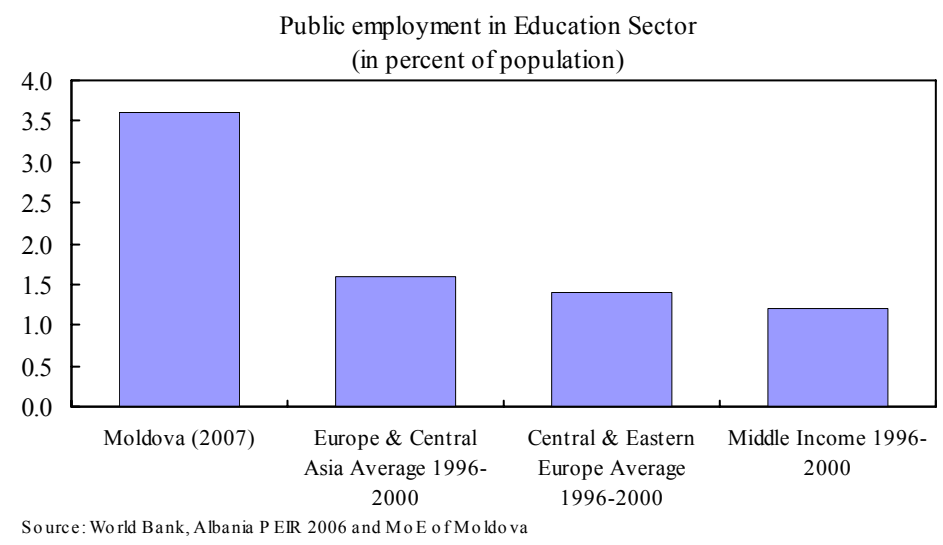




\section{Staff estimate that cutting the CIT rate to zero will reduce revenue by}

$11 / 2$ percent of GDP in 2008. The authorities argue that this step-which mirrors a reform introduced by Estonia in 2000 - would not undermine the fiscal program, given the buoyancy of revenues noted above.

17. The sweeping tax amnesty is risky as it could undermine future tax discipline. All pre-2007 tax arrears are to be written off, whether potentially collectible or not, and audits of pre-2007 returns will be forbidden. The mission - echoing recent FAD TA-argued that this move could weaken tax discipline because it raises expectations of future amnesties and feeds a sense of unfairness among law-abiding taxpayers. The authorities noted that much of the stock of tax debt ( 5 percent of GDP) had accumulated early in transition, and was thus uncollectible.

\section{Staff argued that the President's initiatives could hinder the poverty reduction} and growth objectives of the program. Staff regretted the authorities' intention to reduce public investment, if necessary, to meet the $1 / 2$ percent of GDP deficit target, favoring instead reductions in goods and services spending and in state aid to industry and agriculture. To reduce the risk that tax arrears will develop in the future, the authorities will strengthen tax discipline by modernizing tax arrears management and will eliminate duplication by shifting all tax administration functions from the CCECC to the STI. ${ }^{2}$ A staff visit in September will discuss the draft 2008 budget, passage of which (consistent with the program) will be a focus of the Third Review.

19. The President's capital amnesty could also present risks to the AML regime. The authorities explained they would consult and take into account the views of MONEYVAL and key bilateral partners and take additional steps as needed to ensure that no loopholes arise in their AML framework. Importantly, key revisions to the AML law-developed with LEG TA - are to be presented to parliament this summer.

\section{Financial Sector Stability and Reforms}

\section{The financial sector has proved robust to the continuing difficulties associated} with selling wine to Russia. Financial sector stability indicators appear sound, even though some banks have reportedly rolled over their exposure to the wine sector. The FSAP update — expected during the second half of 2007—will review systemic bank stability in light of the wine ban as well as the supporting regulatory, supervisory and enforcement framework, and measures to promote broader development of the financial system.

\footnotetext{
${ }^{2}$ The authorities have requested FAD TA on tax arrears management.
} 
21. There is growing interest by international banks in the privatization of Banca de Economii, the large state-owned bank. Deloitte \& Touche has been selected to carry out the market evaluation mandated under the program, and the IFC has been approached regarding pre-privatization support.

22. In light of the weak supervision of the nonbank financial sector, the law establishing NCFM was passed in June 2007. The original FSAP noted significant shortcomings in supervision of this sector. Consistent with TA from the Fund and the Netherlands, the law stipulates that upon establishment the NCFM will acquire the right to suspend licenses of market participants in violation of prudential norms, and by endSeptember 2008, it will acquire full licensing authority.

\section{E. Structural Reforms}

23. Improving the targeting of social assistance will bolster the authorities' commitment to the politically-difficult step of raising tariffs for district heat. Increasing effective tariffs to the programmed level of 70 percent of cost recovery by end-September will be challenging because it will require action by a Chişinău city council (to reduce untargeted subsidies) now in the hands of opposition parties. Thus, the methodology establishing a new, proxy means-tested social benefit system - developed with the assistance of DfID, SIDA, and the World Bank - will be approved by government by end-September 2007. Following consultations with civil society, the authorities will begin to introduce the system in January, and it will be fully operational by end-September 2008 (one year earlier than previously expected).

24. The law on divestiture and management of state assets became controversial, because it includes a fairly limited negative list of enterprises that cannot be privatized. Passage of this law was a benchmark for end-December, and it passed in May 2007.

\section{Program Issues}

25. The authorities propose to modify the program by increasing the ceiling on nonconcessional borrowing. This would accommodate an EBRD/EIB road rehabilitation project and a health project by the CEB (both projects are co-financed by IDA). The ceiling would be increased by $€ 60$ million for the roads project and $€ 9$ million for the health project, though disbursements would take place over several years. The DSA indicates that the risk of external debt distress is low and that the evolution of public debt is sustainable. Thus, while the authorities acknowledge that Moldova should primarily seek concessional foreign financing, these potentially high-value projects are affordable over the medium term. Moreover, the authorities are seeking grant co-financing, particularly from the EU, for the second phase of the roads project ( $€ 35$ million) sufficient to attain the 35 percent concessionality threshold for that phase. Staff will report to the Board on this issue in future reviews. 


\section{Staff Appraisal}

26. The macroeconomic outlook is improving as last year's shocks abate. Although it remains unclear when wine exports will resume, there is welcome clarity about the rate at which Russian subsidies for natural gas deliveries will be phased out. Further, Moldova is set to receive significantly higher aid inflows over the coming years. Growth is picking up and inflation is diminishing. With national elections almost two years away, this is an opportune moment to refocus on the key priorities of the PRGF.

27. The NBM should stand ready to tighten monetary policy as needed to ensure achievement of single-digit inflation. Moldova is very dependent on remittances and aid inflows, but they present challenges to monetary policy. The exchange rate should remain flexible and interest rates may need to stay high until inflation is comfortably back in single digits. As they step up sterilization efforts, the authorities will be well served by the NBM's strengthened capital structure, including last December's recapitalization.

\section{Recent tax/capital amnesty initiatives and wage increases threaten to put}

program objectives at risk, particularly in 2008. The 2007 budget outlook is favorable, but over the medium term large public sector wage increases coupled with revenue losses due to CIT reform and the tax amnesty may crowd out investment and compress room for social spending, undermining long-term growth and poverty reduction prospects. These issues will be a focus of the next review. In addition, the capital legalization initiatives could undermine Moldova's commitment to combat money laundering.

29. Compensating measures will therefore be needed. The supplementary budget for 2007 and the 2008 budget should reflect the government's pro-growth, poverty-reduction aims. In this regard, public employment should gradually be downsized and tax administration improvements - including by shifting functions from the CCECC to the STIare essential. The authorities should take steps, such as passage of amendments to the AML law, to ensure that the anti-money laundering regime remains robust.

30. Supervision of the nonbank financial sector is presently inadequate. Creation of the NCFM - with support from international donors and the World Bank-will materially reduce financial sector risks. However, to ensure the new institution is effective, it must have the effective right to issue and withdraw licenses.

31. Better targeting of subsidies for district heating will be important to ensure that municipal finances remain sound. Introduction of a targeted social assistance system will allow the authorities to reduce these subsidies. In the interim, tariffs actually paid by households should gradually rise to ensure the adjustment is not abrupt.

32. In recommending completion of the second review, staff supports the authorities' request for a waiver of non-observance of a performance criterion. The non-observance of the $\mathrm{PC}$ was temporary and the authorities continue to demonstrate strong 
program ownership. Their commitment, and the support of the international community, provide assurance that economic growth and poverty reduction will continue. While Moldova should primarily seek concessional foreign financing, the favorable DSA provides limited room for non-concessional borrowing. 
Figure 1. Moldova: PRGF Performance, 2007

(In millions of lei, unless indicated otherwise)
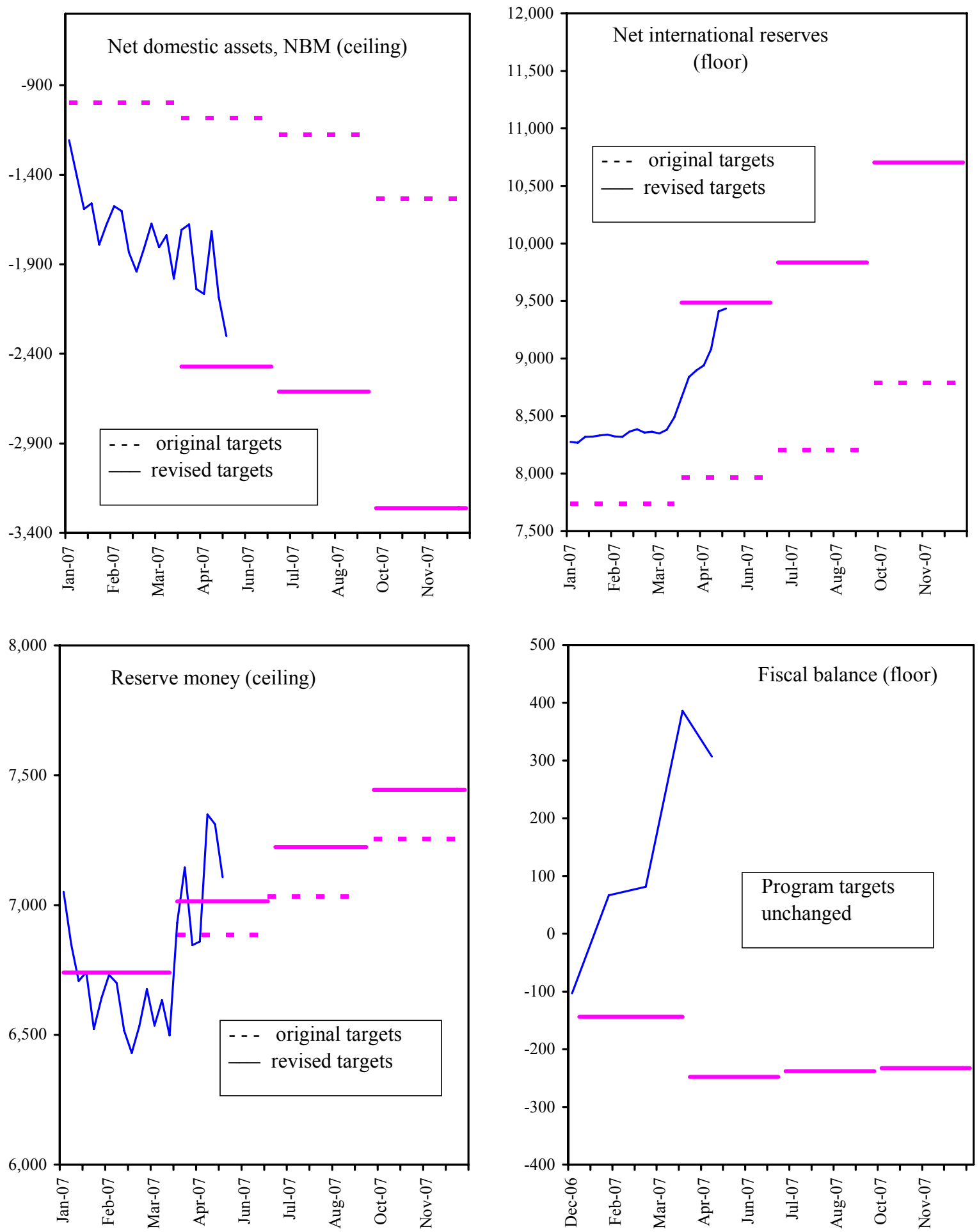

Sources: Moldovan authorities; and Fund staff calculations. 
Figure 2. Moldova: Inflation

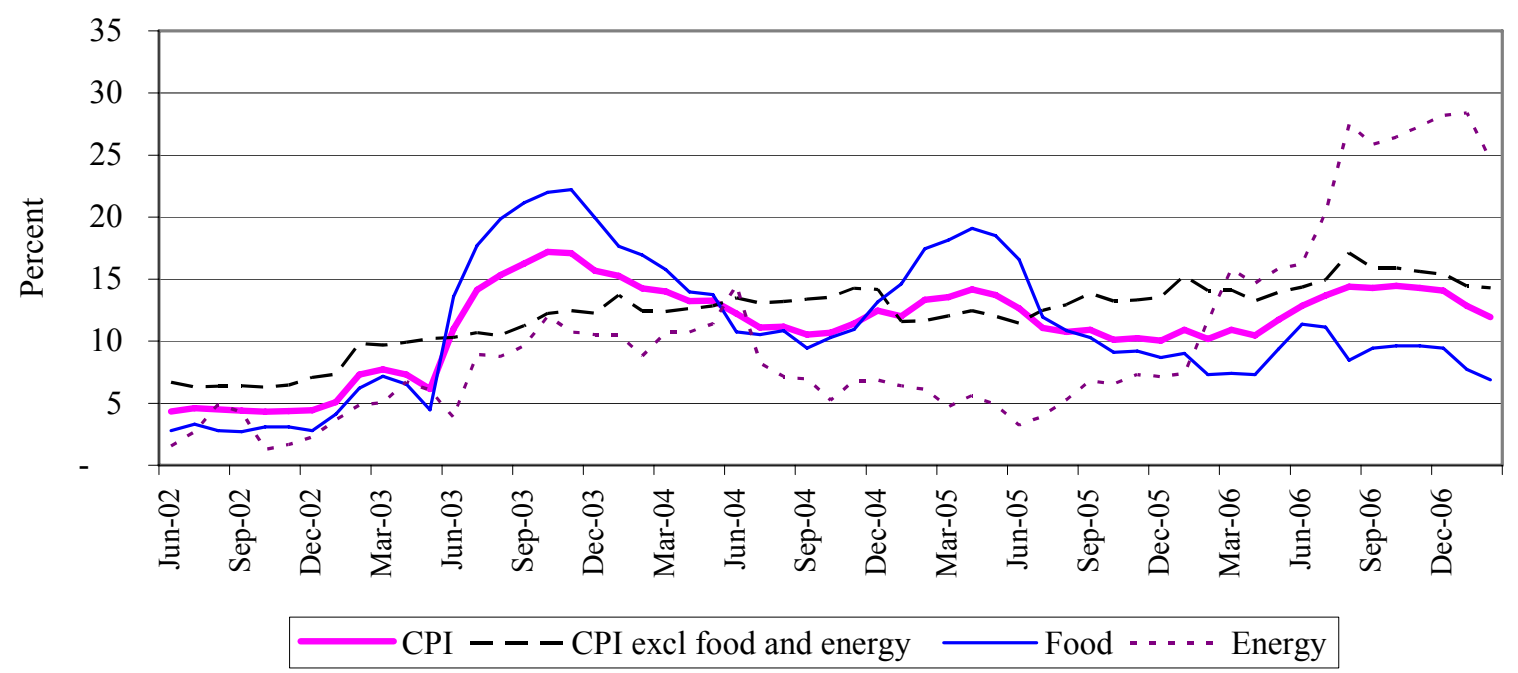

Inflation in Moldova and Neighbouring Regions 1/

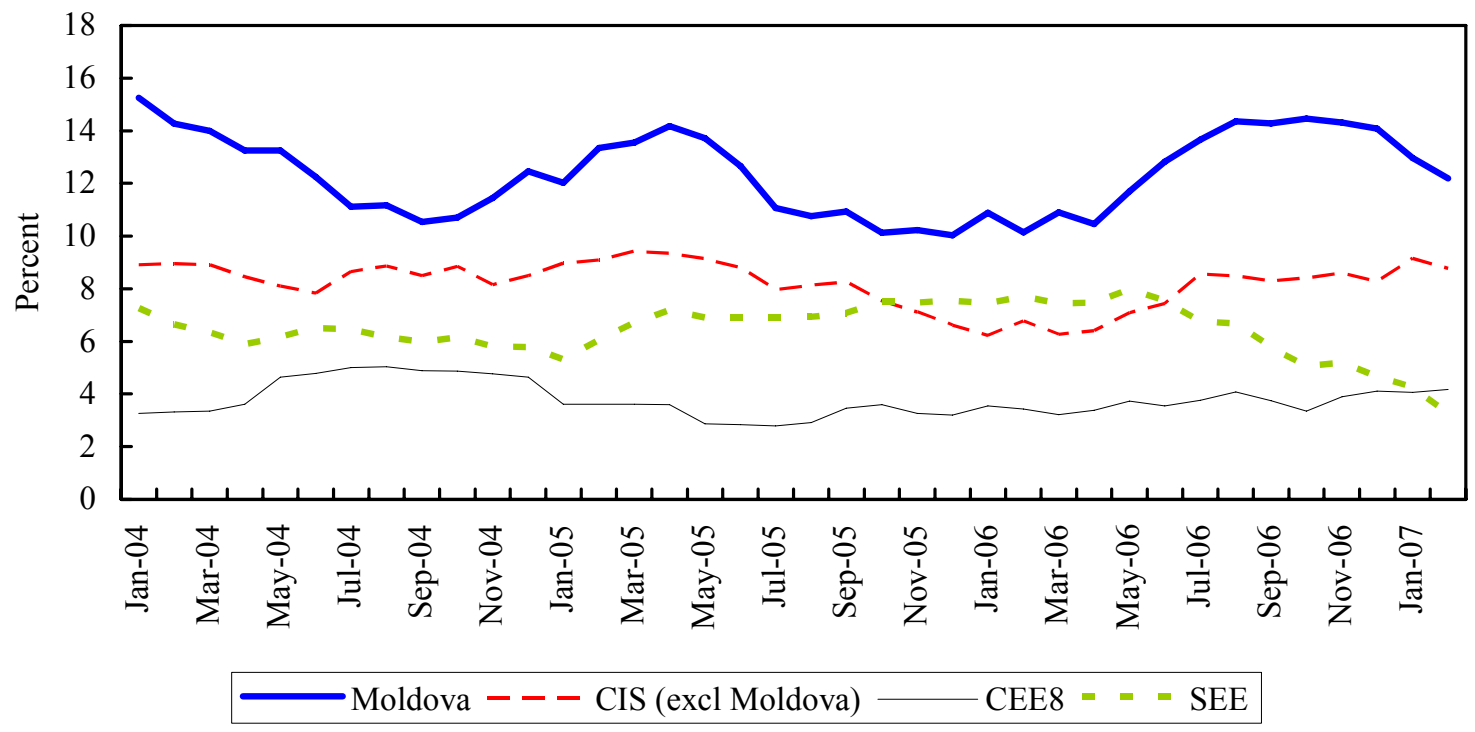

1/ CIS excludes Turkmenistan, Uzbekistan and Tajikistan

CEE8 includes Czech Rep, Hungary, Poland, Slovakia, Slovenia and Baltics

SEE includes Romania, Bulgaria, Macedonia, Serbia and Montenegro, and Croatia

Source: Moldovan authorities; and Fund staff estimates and calculations. 
Figure 3. Moldova: Liquidity Conditions

Leu/U.S. Dollar Exchange Rate and Foreign Exchange Purchases by the Central Bank, 2004-06

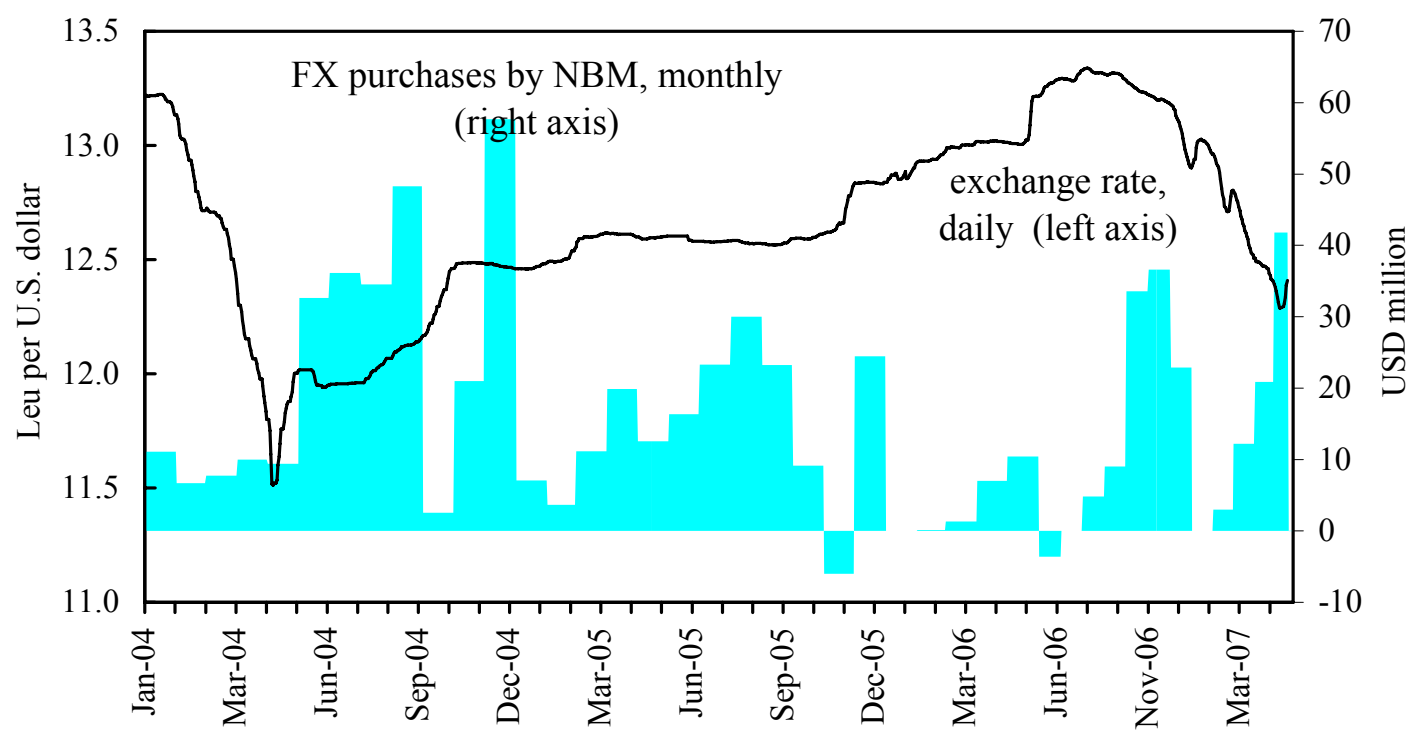

Interest Rates and Central Bank Sterilisation Operations

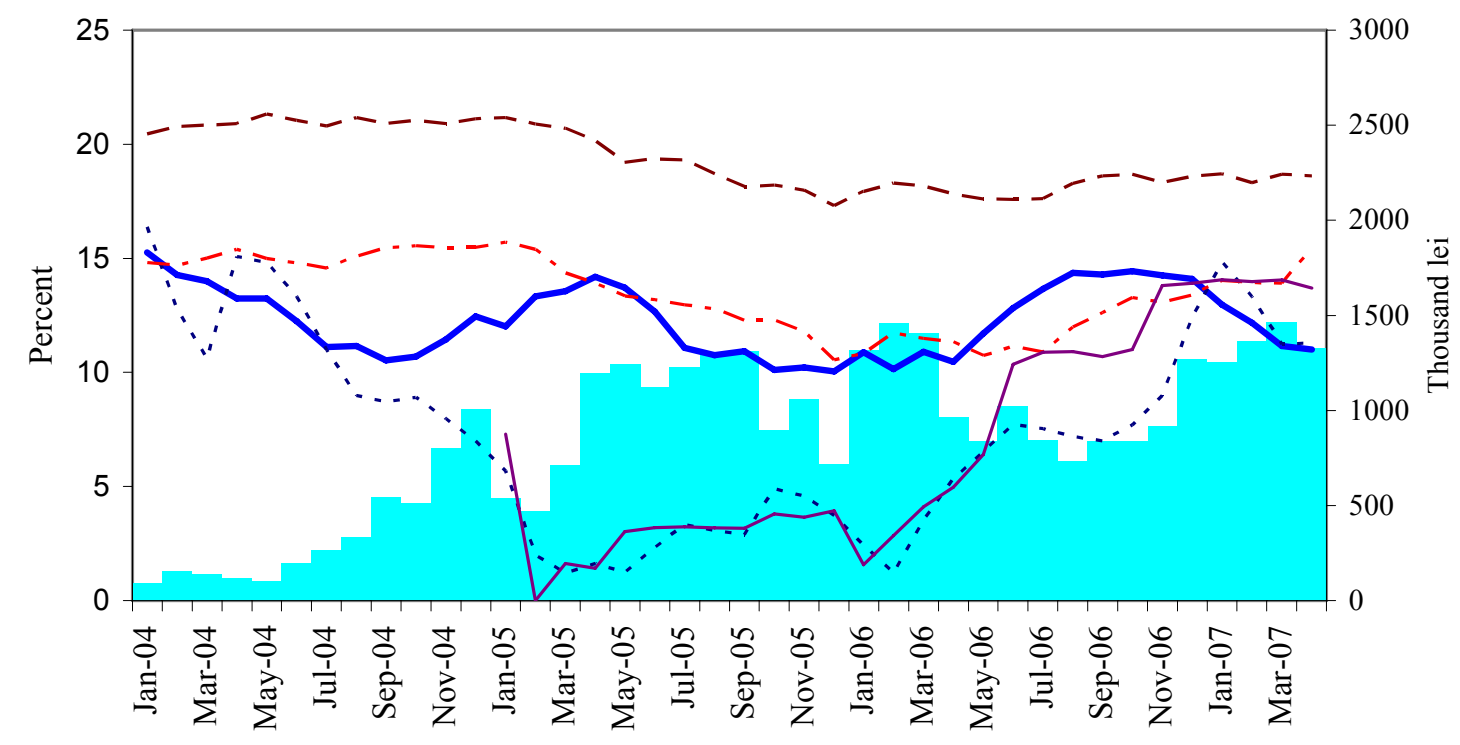

Sterilisation balance (rhs) - Inflation
-- - - Deposits

-. . 91-day T-bills

NBM certificates

Source: Moldovan authorities. 
Figure 4. Moldova: Monetary Developments
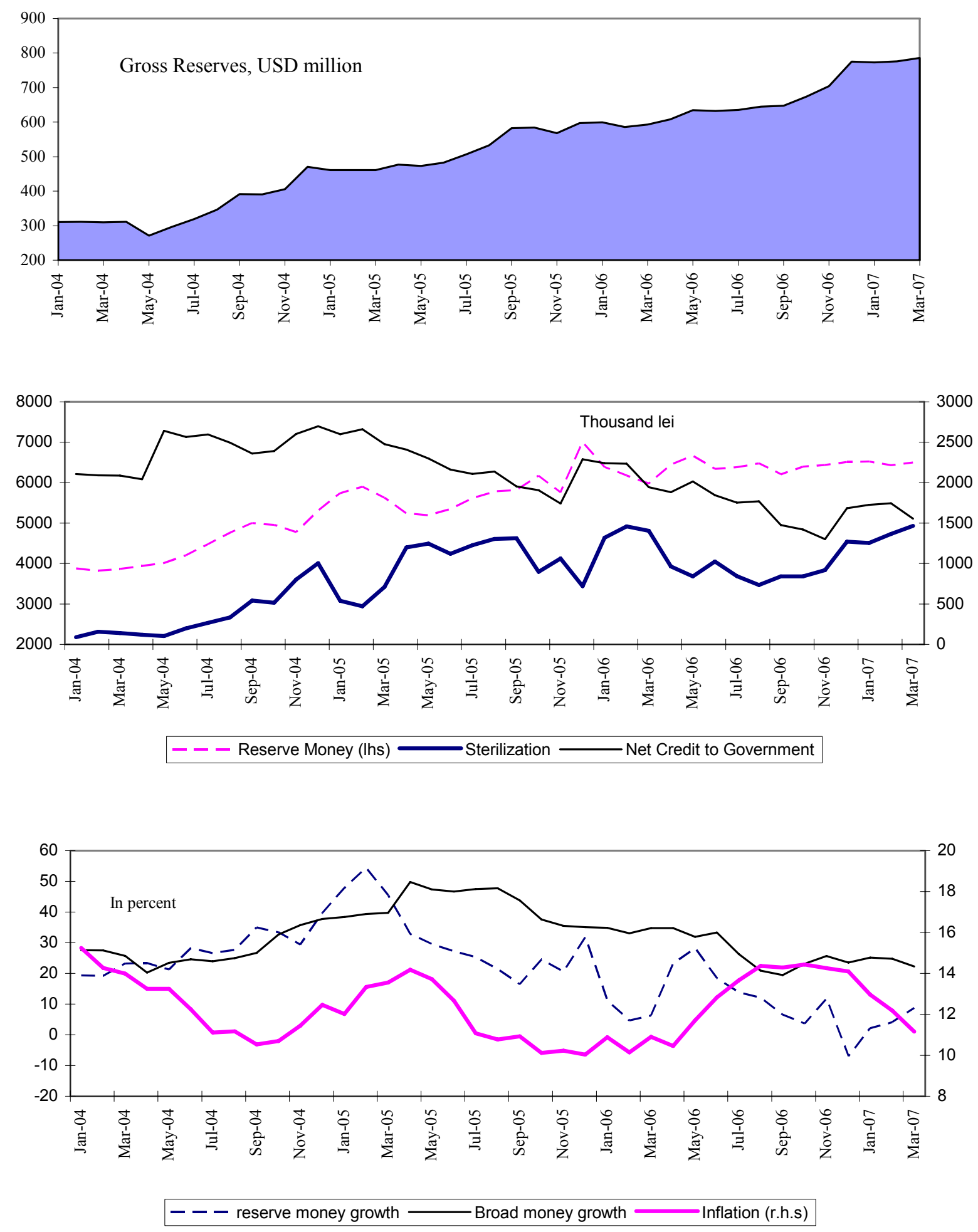

Sources: Moldovan authorities; and Fund staff estimates. 
Table 1. Moldova: Selected Indicators, 2004-09 1/

\begin{tabular}{|c|c|c|c|c|c|c|c|c|c|}
\hline & \multirow[t]{2}{*}{2004} & \multirow[t]{2}{*}{2005} & \multicolumn{2}{|l|}{2006} & \multicolumn{2}{|c|}{2007} & \multicolumn{2}{|c|}{2008} & \multirow{2}{*}{$\begin{array}{l}2009 \\
\text { Proj }\end{array}$} \\
\hline & & & $\begin{array}{c}\text { IMF CR } \\
\text { No. } 07 / 45\end{array}$ & Prel. & $\begin{array}{c}\text { IMF CR } \\
\text { No. } 07 / 45\end{array}$ & Proj. & $\begin{array}{c}\text { IMF CR } \\
\text { No. } 07 / 45\end{array}$ & Proj. & \\
\hline I. Real sector indicators & \multicolumn{9}{|c|}{ (Percent change; unless otherwise indicated) } \\
\hline \multicolumn{10}{|l|}{ Gross domestic product } \\
\hline Real growth rate & 7.4 & 7.5 & 3.0 & 4.0 & 3.0 & 5.0 & 5.0 & 5.0 & 5.0 \\
\hline Nominal GDP (millions of lei) & 32,032 & 37,652 & 41,953 & 44,069 & 46,603 & 49,708 & 53,195 & 56,039 & 62,850 \\
\hline Nominal GDP (millions of U.S. dollars) & 2,598 & 2,988 & 3,166 & 3,356 & 3,189 & 3,983 & 3,441 & 4,607 & 5,213 \\
\hline Consumer Price Index (average) & 12.5 & 11.9 & 12.3 & 12.7 & 10.5 & 10.6 & 8.9 & 9.3 & 7.5 \\
\hline Consumer Price Index (end of period) & 12.6 & 10.1 & 12.0 & 14.1 & 10.0 & 10.0 & 8.0 & 8.0 & 7.0 \\
\hline GDP deflator & 8.0 & 9.3 & 10.8 & 12.5 & 7.8 & 7.4 & 8.7 & 7.4 & 6.8 \\
\hline Average monthly wage (lei) & 1,116 & 1,320 & $\ldots$ & 1695 & $\ldots$ & $\ldots$ & $\ldots$ & $\ldots$ & $\ldots$ \\
\hline Average monthly wage (U.S. dollars) & 91 & 105 & $\ldots$ & 129 & $\ldots$ & $\ldots$ & $\ldots$ & $\ldots$ & $\ldots$ \\
\hline Saving-investment balance & \multicolumn{9}{|c|}{ (In percent of GDP) } \\
\hline Foreign saving & 2.0 & 10.3 & 10.4 & 12.0 & 7.2 & 8.9 & 4.2 & 8.2 & 7.1 \\
\hline National saving & 19.2 & 14.3 & 13.0 & 15.9 & 16.2 & 18.9 & 18.8 & 20.2 & 21.9 \\
\hline Private & 13.6 & 6.8 & 7.4 & 7.7 & 10.5 & 12.4 & 13.8 & 13.7 & 16.3 \\
\hline Public & 5.5 & 7.5 & 5.6 & 8.2 & 5.7 & 6.5 & 5.0 & 6.5 & 5.6 \\
\hline Gross investment & 21.2 & 24.6 & 23.5 & 27.9 & 23.4 & 27.7 & 23.1 & 28.4 & 29.0 \\
\hline Private & 16.2 & 18.4 & 17.9 & 20.0 & 17.2 & 20.8 & 17.6 & 21.4 & 22.0 \\
\hline Public & 5.0 & 6.2 & 5.5 & 8.0 & 6.2 & 7.0 & 5.5 & 7.0 & 7.0 \\
\hline II. Fiscal Indicators (general government) & \multicolumn{9}{|c|}{ (In percent of GDP) } \\
\hline Primary balance (cash) & 2.7 & 2.6 & 1.3 & 1.3 & 1.0 & 0.8 & 0.7 & 0.6 & 0.5 \\
\hline Overall balance (cash) & 0.8 & 1.3 & 0.0 & 0.3 & -0.5 & -0.5 & -0.5 & -0.5 & -0.5 \\
\hline Overall balance (commitments) & 0.5 & 1.3 & 0.1 & 0.3 & -0.5 & -0.5 & -0.5 & -0.5 & -0.5 \\
\hline $\begin{array}{l}\text { Overall balance (commitments), excluding foreign-fir } \\
\text { projects }\end{array}$ & 20 & 22. & 1.0 & 12 & 10 & 07 & 03 & 10 & 08 \\
\hline Stock of general government debt & 47.2 & 38.0 & $\begin{array}{r}1.0 \\
32.8\end{array}$ & $\begin{array}{r}1.2 \\
34.6\end{array}$ & $\begin{array}{r}1.0 \\
32.2\end{array}$ & $\begin{array}{r}0.1 \\
31.1\end{array}$ & $\begin{array}{r}0.3 \\
29.5\end{array}$ & $\begin{array}{r}1.0 \\
26.0\end{array}$ & $\begin{array}{r}0.8 \\
22.8\end{array}$ \\
\hline III. Financial indicators & \multicolumn{9}{|c|}{ (Percent change; unless otherwise indicated) } \\
\hline Broad money (M3) & 37.7 & 35.0 & 19.4 & 23.6 & 14.7 & 18.2 & $\ldots$ & $\ldots$ & $\ldots$ \\
\hline Velocity (GDP/end-period M3; ratio) & 2.7 & 2.4 & 2.2 & 2.3 & 2.2 & 2.2 & $\ldots$ & $\cdots$ & $\cdots$ \\
\hline Reserve money & 39.7 & 31.8 & -5.6 & -7.0 & 9.7 & 14.3 & $\ldots$ & $\ldots$ & $\ldots$ \\
\hline Credit to the economy & 22.2 & 35.0 & 26.1 & 37.8 & 13.3 & 17.4 & $\ldots$ & $\ldots$ & $\ldots$ \\
\hline IV. External sector indicators & \multicolumn{9}{|c|}{ (In millions of U.S. dollars, unless otherwise indicated) } \\
\hline Current account balance & -52.9 & -306.9 & -330.7 & -403.7 & -229.4 & -353.5 & -145.4 & -379.4 & -369.9 \\
\hline Current account balance (percent of GDP) & -2.0 & -10.3 & -10.4 & -12.0 & -7.2 & -8.9 & -4.2 & -8.2 & -7.1 \\
\hline Remittances and compensation of employees (net) & 660 & 869 & 1,156 & 1,119 & 1,283 & 1,343 & 1,411 & 1,531 & 1,743 \\
\hline Gross official reserves $2 /$ & 470 & 597 & 688 & 775 & 799 & 994 & 962 & 1,264 & 1,595 \\
\hline Gross official reserves (months of imports) & 2.1 & 2.2 & 2.3 & 2.5 & 2.4 & 2.9 & 2.7 & 3.3 & 3.9 \\
\hline Exchange rate $(\mathrm{MDL} / \$)$ period average & 12.3 & 12.6 & 13.3 & 13.1 & $\ldots$ & $\ldots$ & $\ldots$ & $\ldots$ & $\ldots$ \\
\hline Exchange rate $(\mathrm{MDL} / \$)$ end of period & 12.5 & 12.8 & 13.8 & 12.9 & $\cdots$ & $\cdots$ & $\cdots$ & $\begin{array}{l}\cdots \\
\ldots\end{array}$ & $\cdots$ \\
\hline Real effective exchange rate, change (percent) & 12.8 & -1.3 & 1.8 & 0.0 & $\ldots$ & $\ldots$ & $\ldots$ & $\ldots$ & $\ldots$ \\
\hline External debt/GDP (percent) 3/ & 63.8 & 53.4 & 51.6 & 53.3 & 55.1 & 49.1 & 53.7 & 43.3 & 39.1 \\
\hline Debt service/exports of goods and services (percent) & 21.0 & 20.3 & 16.7 & 16.0 & 14.4 & 13.1 & 14.1 & 13.2 & 13.8 \\
\hline \multicolumn{10}{|l|}{ Social indicators, reference year } \\
\hline Per capita GNI, U.S. dollars: $880(2005)$ & \multicolumn{9}{|c|}{ Population below poverty line, percent: $30.2(2005)$} \\
\hline Life expectancy, male, years: $64.9(2005)$ & \multicolumn{9}{|c|}{ Life expectancy, female, years: 71.9 (2005) } \\
\hline
\end{tabular}

Sources: Moldovan authorities; and Fund staff estimates.

1/ Data exclude Transnistria.

2/ For 2005, includes the positive effect of reclassification of $\$ 43$ million.

$3 /$ Includes private and public debt. 
Table 2. Moldova: Balance of Payments, 2005-09

(In millions of U.S. dollars; unless otherwise indicated)

\begin{tabular}{|c|c|c|c|c|c|c|c|c|}
\hline & \multirow[t]{2}{*}{2005} & \multicolumn{2}{|c|}{2006} & \multicolumn{2}{|c|}{2007} & \multicolumn{2}{|c|}{2008} & \multirow{2}{*}{$\frac{2009}{\text { Proj. }}$} \\
\hline & & $\begin{array}{c}\text { IMF CR } \\
\text { No. 07/45 }\end{array}$ & Prel. & $\begin{array}{c}\text { IMF CR } \\
\text { No. } 07 / 45\end{array}$ & Proj. & $\begin{array}{c}\text { IMF CR } \\
\text { No. 07/45 }\end{array}$ & Proj. & \\
\hline Current account & -306.9 & -330.7 & -403.7 & -229.4 & -353.6 & -145.4 & -379.4 & -369.9 \\
\hline Merchandise trade balance & $-1,190.8$ & $-1,667.6$ & $-1,590.9$ & $-1,827.4$ & $-1,907.7$ & $-1,945.3$ & $-2,173.6$ & $-2,401.9$ \\
\hline Exports of goods & $1,104.4$ & $1,042.7$ & $1,053.5$ & $1,194.9$ & $1,208.6$ & $1,345.0$ & $1,373.6$ & $1,550.4$ \\
\hline of which: wine and alcohol & 314.7 & 150.4 & 186.8 & 195.5 & 226.1 & 245.7 & 294.0 & 374.9 \\
\hline Imports of goods & $-2,295.2$ & $-2,710.4$ & $-2,644.4$ & $-3,022.3$ & $-3,116.3$ & $-3,290.3$ & $-3,547.2$ & $-3,952.3$ \\
\hline of which: energy & -426.4 & -608.0 & -544.9 & -730.7 & -637.8 & -769.5 & -706.4 & -742.2 \\
\hline non-energy & $-1,868.8$ & $-2,102.4$ & $-2,099.5$ & $-2,291.6$ & $-2,478.5$ & $-2,520.8$ & $-2,840.8$ & $-3,210.1$ \\
\hline Balance of services & -20.2 & -6.9 & -6.0 & 21.4 & -6.7 & 38.7 & -10.0 & -10.4 \\
\hline Exports of services & 423.9 & 517.2 & 482.5 & 592.7 & 540.4 & 667.1 & 567.4 & 595.7 \\
\hline Imports of services & -444.2 & -524.1 & -488.5 & -571.3 & -547.1 & -628.4 & -577.3 & -606.2 \\
\hline Income (net) & 358.8 & 543.5 & 399.6 & 603.5 & 515.2 & 677.9 & 593.5 & 695.7 \\
\hline Compensation of employees & 476.8 & 634.2 & 522.6 & 703.9 & 627.1 & 774.3 & 714.9 & 814.0 \\
\hline Income on direct and portfolio investment & -91.7 & -63.3 & -109.4 & -63.8 & -80.0 & -68.8 & -93.6 & -105.9 \\
\hline Income on other investment & -26.3 & -27.3 & -13.6 & -36.6 & -31.9 & -27.6 & -27.8 & -12.4 \\
\hline Current transfers (net), of which: & 545.3 & 800.3 & 793.7 & 973.0 & $1,045.7$ & $1,083.2$ & $1,210.6$ & $1,346.8$ \\
\hline Remittances, net & 392.0 & 521.3 & 596.4 & 578.7 & 715.6 & 636.5 & 815.8 & 928.9 \\
\hline Official transfers, of which: & 45.7 & 62.1 & 58.5 & 124.1 & 168.5 & 135.3 & 218.3 & 225.6 \\
\hline Budget grants & $\ldots$ & 9.6 & 24.0 & 52.7 & 84.3 & 57.5 & 120.1 & 128.6 \\
\hline Capital and financial account & 211.1 & 276.8 & 271.6 & 287.5 & 544.9 & 267.1 & 636.1 & 701.6 \\
\hline Capital account & -16.9 & -8.0 & -22.8 & -8.0 & -22.8 & -8.0 & -22.8 & -15.0 \\
\hline Financial account & 228.0 & 284.8 & 294.4 & 295.5 & 567.7 & 275.1 & 658.9 & 716.6 \\
\hline Direct investment & 225.5 & 221.6 & 223.0 & 223.3 & 350.8 & 240.9 & 414.6 & 469.2 \\
\hline Portfolio investment (net) & -7.0 & -9.8 & -4.7 & -10.0 & -4.7 & -10.0 & -4.7 & -4.7 \\
\hline Other investments & 11.0 & 73.0 & 75.8 & 82.3 & 221.4 & 44.2 & 248.8 & 252.1 \\
\hline Other capital flows & -10.1 & 20.0 & 25.9 & 20.0 & 100.0 & 20.0 & 100.0 & 100.0 \\
\hline Errors and omissions $1 /$ & 192.1 & 73.0 & 117.3 & 0.0 & 0.0 & 0.0 & 0.0 & 0.0 \\
\hline Overall balance & 96.3 & 19.1 & -14.8 & 58.1 & 191.4 & 121.7 & 256.7 & 331.7 \\
\hline Financing & -96.3 & -48.8 & 14.8 & -72.8 & -191.4 & -125.4 & -256.7 & -331.7 \\
\hline Use of Fund credit 2/ & -21.4 & 9.2 & 41.1 & 11.9 & $\begin{array}{r}27.5 \\
-2189\end{array}$ & 12.9 & 13.3 & -0.52 \\
\hline Change of gross official reserves (increase -) & -128.6 & -63.6 & -140.8 & -110.8 & -218.9 & -163.1 & -270.0 & -331.7 \\
\hline Exceptional financing: & 53.8 & 5.5 & 114.5 & 26.1 & 0.0 & 24.8 & 0.0 & 0.0 \\
\hline Operations with PPG debt & 16.9 & 5.5 & 26.8 & 26.1 & 0.0 & 24.8 & 0.0 & 0.0 \\
\hline Accumulation of arrears on public debt & 17.9 & 11.9 & 26.1 & 0.0 & 0.0 & 0.0 & 0.0 & 0.0 \\
\hline Clearance of arrears & -1.0 & -68.1 & -87.0 & 0.0 & 0.0 & 0.0 & 0.0 & 0.0 \\
\hline Prepayment/buyback & 0.0 & 0.0 & 0.0 & 0.0 & 0.0 & 0.0 & 0.0 & 0.0 \\
\hline Cancellation of debt & 0.0 & 0.0 & 0.0 & 0.0 & 0.0 & 0.0 & 0.0 & 0.0 \\
\hline Rescheduling, net of arrears repayment & 0.0 & 61.7 & 87.7 & 26.1 & 0.0 & 24.8 & 0.0 & 0.0 \\
\hline Operations with private debt & 1.9 & 0.0 & 83.0 & 0.0 & 0.0 & 0.0 & 0.0 & 0.0 \\
\hline o.w. accumulation of arrears on energy debt & 0.0 & 0.0 & 34.0 & 0.0 & 0.0 & 0.0 & 0.0 & 0.0 \\
\hline Transfers & 35.0 & $\ldots$ & 4.7 & $\ldots$ & 0.0 & $\ldots$ & 0.0 & 0.0 \\
\hline Financing gap & $\ldots$ & 29.7 & 0.0 & 14.7 & 0.0 & 3.7 & 0.0 & 0.0 \\
\hline $\begin{array}{l}\text { Memorandum items: } \\
\text { Gross official reserves } 3 / \\
\text { in months of imports of goods and services }\end{array}$ & $\begin{array}{r}597.4 \\
2.3\end{array}$ & $\begin{array}{r}688.4 \\
2.3\end{array}$ & $\begin{array}{r}775.3 \\
2.5\end{array}$ & $\begin{array}{r}799.3 \\
2.4\end{array}$ & $\begin{array}{r}994.2 \\
2.9\end{array}$ & $\begin{array}{r}962.4 \\
2.7\end{array}$ & $\begin{array}{r}1,264.2 \\
3.3\end{array}$ & $\begin{array}{r}1,595.4 \\
3.9\end{array}$ \\
\hline IMF augmentation (Dec 2006) & $\ldots$ & 29.7 & $\ldots$ & 14.7 & $\ldots$ & $\ldots$ & $\ldots$ & \\
\hline Nominal GDP & $2,988.3$ & $3,166.2$ & $3,356.2$ & $3,189.3$ & $3,982.8$ & $3,441.3$ & $4,606.7$ & $5,213.3$ \\
\hline Current account balance (percent of GDP) & -10.3 & -10.4 & -12.0 & -7.2 & -8.8 & -4.2 & -8.2 & -7.1 \\
\hline G\&S trade balance (percent of GDP) & -40.5 & -52.9 & -47.6 & -56.6 & -48.1 & -55.4 & -47.4 & -46.3 \\
\hline Exports G\&S (percent of GDP) & 51.1 & 49.3 & 45.8 & 56.0 & 43.9 & 58.5 & 42.1 & 41.2 \\
\hline Exports G\&S growth (percent) & 14.8 & 2.1 & 0.5 & 14.6 & 13.9 & 12.6 & 11.0 & 10.6 \\
\hline Imports G\&S (percent of GDP) & -91.7 & -102.2 & -93.3 & -112.7 & -92.0 & -113.9 & -89.5 & -87.4 \\
\hline Imports G\&S growth (percent) & 29.7 & 18.1 & 14.4 & 11.1 & 16.9 & 9.0 & 12.6 & 10.5 \\
\hline Remittances and compensation of employees (net) & 868.8 & $1,155.5$ & $1,119.0$ & $1,282.6$ & $1,342.8$ & $1,410.9$ & $1,530.7$ & $1,742.9$ \\
\hline Total debt service/exports G\&S (percent) & 20.3 & 16.7 & 16.0 & 14.4 & 13.1 & 14.1 & 13.2 & 13.8 \\
\hline External debt stock (percent of GDP) 4/ & 53.4 & 51.6 & 53.3 & 55.1 & 49.1 & 53.7 & 43.3 & 39.1 \\
\hline Natural gas import price, $\$ / 1000 \mathrm{~m} 3$ & 68.8 & 132.9 & 120.0 & 220.0 & 170.0 & 220.0 & 185.4 & 190.3 \\
\hline External energy arrears & 286.6 & 286.6 & 320.5 & 286.6 & 320.5 & 286.6 & 320.5 & 320.5 \\
\hline External PPG arrears & 56.1 & 0.0 & 0.0 & 0.0 & 0.0 & 0.0 & 0.0 & 0.0 \\
\hline
\end{tabular}

Sources: National Bank of Moldova; and Fund staff estimates and projections.

1/ Revised projection for 2006 shows the actual number for the first half of the year.

2/ Includes prospective disbursements.

3/ Includes revaluation changes, which are not captured by changes of gross official reserves in the BoP.

4/ Including energy arrears. 
Table 3. Moldova: General Government Budget, 2004-09

(In millions of lei; unless otherwise indicated)

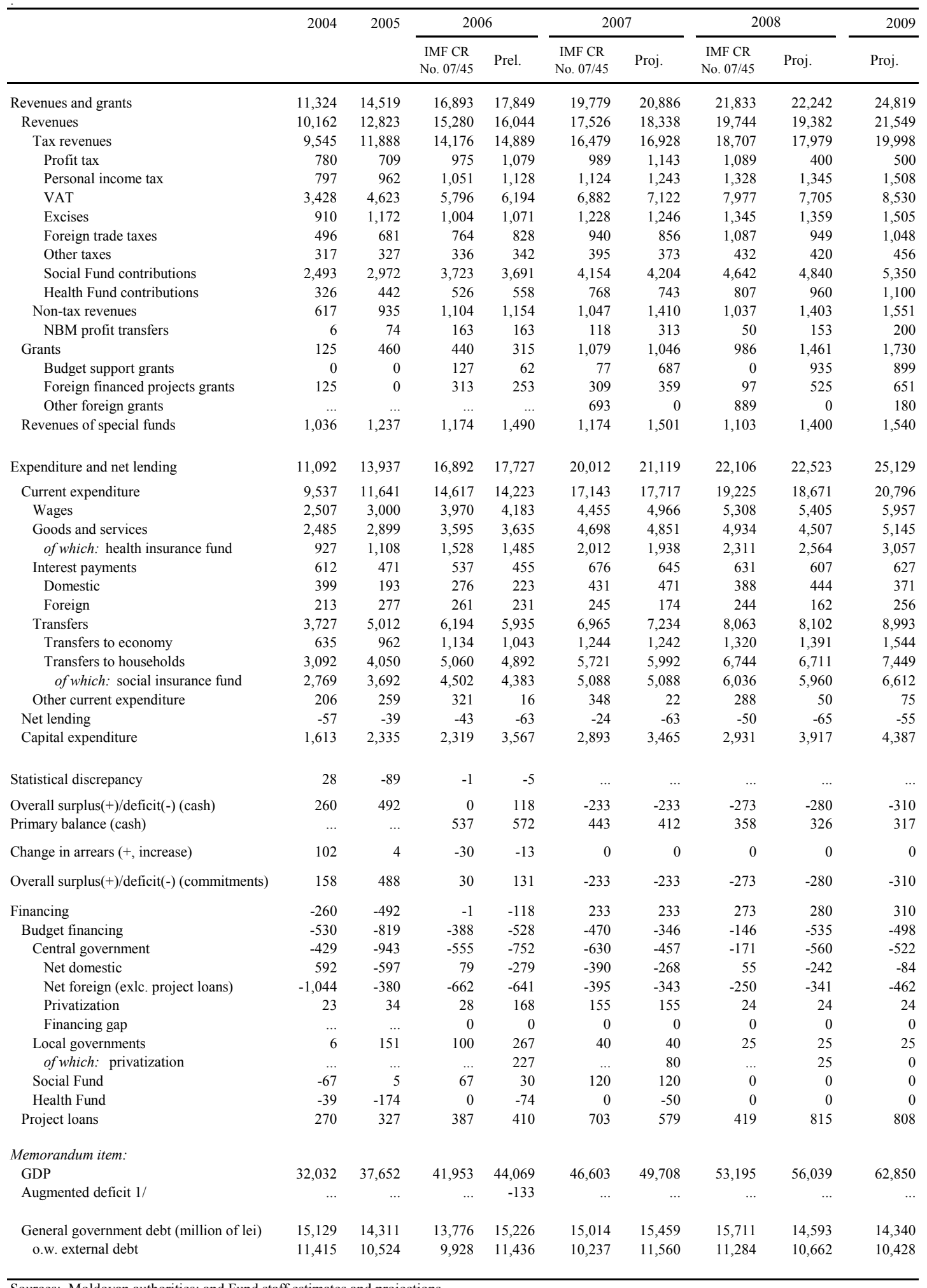

1/ Includes lei $250 \mathrm{mln}$ of NBM recapitalization. 
Table 3 (continued). Moldova: General Government Budget, 2004-09 (In percent of GDP; unless otherwise indicated)

\begin{tabular}{|c|c|c|c|c|c|c|c|c|c|}
\hline & \multirow[t]{2}{*}{2004} & \multirow[t]{2}{*}{2005} & \multicolumn{2}{|c|}{2006} & \multicolumn{2}{|c|}{2007} & \multicolumn{2}{|c|}{2008} & \multirow{2}{*}{$\frac{2009}{\text { Proj. }}$} \\
\hline & & & $\begin{array}{c}\text { IMF CR } \\
\text { No. } 07 / 45\end{array}$ & Prel. & $\begin{array}{c}\text { IMF CR } \\
\text { No. 07/45 }\end{array}$ & Proj. & $\begin{array}{c}\text { IMF CR } \\
\text { No. } 07 / 45\end{array}$ & Proj. & \\
\hline Revenues and grants & 35.4 & 38.6 & 40.3 & 40.5 & 42.4 & 42.0 & 41.0 & 39.7 & 39.5 \\
\hline Revenues & 31.7 & 34.1 & 36.4 & 36.4 & 37.6 & 36.9 & 37.1 & 34.6 & 34.3 \\
\hline Tax revenues & 29.8 & 31.6 & 33.8 & 33.8 & 35.4 & 34.1 & 35.2 & 32.1 & 31.8 \\
\hline Profit tax & 2.4 & 1.9 & 2.3 & 2.4 & 2.1 & 2.3 & 2.0 & 0.7 & 0.8 \\
\hline Personal income tax & 2.5 & 2.6 & 2.5 & 2.6 & 2.4 & 2.5 & 2.5 & 2.4 & 2.4 \\
\hline VAT & 10.7 & 12.3 & 13.8 & 14.1 & 14.8 & 14.3 & 15.0 & 13.7 & 13.6 \\
\hline Excises & 2.8 & 3.1 & 2.4 & 2.4 & 2.6 & 2.5 & 2.5 & 2.4 & 2.4 \\
\hline Foreign trade taxes & 1.5 & 1.8 & 1.8 & 1.9 & 2.0 & 1.7 & 2.0 & 1.7 & 1.7 \\
\hline Other taxes & 1.0 & 0.9 & 0.8 & 0.8 & 0.8 & 0.8 & 0.8 & 0.8 & 0.7 \\
\hline Social Fund contributions & 7.8 & 7.9 & 8.9 & 8.4 & 8.9 & 8.5 & 8.7 & 8.6 & 8.5 \\
\hline Health Fund contributions & 1.0 & 1.2 & 1.3 & 1.3 & 1.6 & 1.5 & 1.5 & 1.7 & 1.8 \\
\hline Non-tax revenues & 1.9 & 2.5 & 2.6 & 2.6 & 2.2 & 2.8 & 1.9 & 2.5 & 2.5 \\
\hline NBM profit transfers & 0.0 & 0.2 & 0.4 & 0.4 & 0.3 & 0.6 & 0.1 & 0.3 & 0.3 \\
\hline Grants & 0.4 & 1.2 & 1.0 & 0.7 & 2.3 & 2.1 & 1.9 & 2.6 & 2.8 \\
\hline Budget support grants & 0.0 & 0.0 & 0.3 & 0.1 & 0.2 & 1.4 & 0.0 & 1.7 & 1.4 \\
\hline Foreign financed projects grants & 0.4 & 0.0 & 0.7 & 0.6 & 0.7 & 0.7 & 0.2 & 0.9 & 1.0 \\
\hline Other foreign grants & $\ldots$ & $\ldots$ & $\ldots$ & $\cdots$ & 1.5 & 0.0 & 1.7 & 0.0 & 0.3 \\
\hline Revenues of special funds & 3.2 & 3.3 & 2.8 & 3.4 & 2.5 & 3.0 & 2.1 & 2.5 & 2.5 \\
\hline Expenditure and net lending & 34.6 & 37.0 & 40.3 & 40.2 & 42.9 & 42.5 & 41.6 & 40.2 & 40.0 \\
\hline Current expenditure & 29.8 & 30.9 & 34.8 & 32.3 & 36.8 & 35.6 & 36.1 & 33.3 & 33.1 \\
\hline Wages & 7.8 & 8.0 & 9.5 & 9.5 & 9.6 & 10.0 & 10.0 & 9.6 & 9.5 \\
\hline Goods and services & 7.8 & 7.7 & 8.6 & 8.2 & 10.1 & 9.8 & 9.3 & 8.0 & 8.2 \\
\hline Interest payments & 1.9 & 1.2 & 1.3 & 1.0 & 1.5 & 1.3 & 1.2 & 1.1 & 1.0 \\
\hline Transfers & 11.6 & 13.3 & 14.8 & 13.5 & 14.9 & 14.6 & 15.2 & 14.5 & 14.3 \\
\hline Other current expenditure & 0.6 & 0.7 & 0.8 & 0.0 & 0.7 & 0.0 & 0.5 & 0.1 & 0.1 \\
\hline Net lending & -0.2 & -0.1 & -0.1 & -0.1 & -0.1 & -0.1 & -0.1 & -0.1 & -0.1 \\
\hline Capital expenditure & 5.0 & 6.2 & 5.5 & 8.1 & 6.2 & 7.0 & 5.5 & 7.0 & 7.0 \\
\hline Statistical discrepancy & 0.1 & -0.2 & 0.0 & 0.0 & $\ldots$ & $\cdots$ & $\ldots$ & $\cdots$ & $\cdots$ \\
\hline Overall surplus(+)/deficit(-) (cash) & 0.8 & 1.3 & 0.0 & 0.3 & -0.5 & -0.5 & -0.5 & -0.5 & -0.5 \\
\hline Primary balance (cash) & 2.7 & 2.6 & 1.3 & 1.3 & 1.0 & 0.8 & 0.7 & 0.6 & 0.5 \\
\hline Change in arrears $(+$, increase $)$ & 0.3 & 0.0 & -0.1 & 0.0 & 0.0 & 0.0 & 0.0 & 0.0 & 0.0 \\
\hline Overall surplus(+)/deficit(-) (commitment: & 0.5 & 1.3 & 0.1 & 0.3 & -0.5 & -0.5 & -0.5 & -0.5 & -0.5 \\
\hline Financing & -0.8 & -1.3 & 0.0 & -0.3 & 0.5 & 0.5 & 0.5 & 0.5 & 0.5 \\
\hline Budget financing & -1.7 & -2.2 & -0.9 & -1.2 & -1.0 & -0.7 & -0.3 & -1.0 & -0.8 \\
\hline Central government & -1.3 & -2.5 & -1.3 & -1.7 & -1.4 & -0.9 & -0.3 & -1.0 & -0.8 \\
\hline Net domestic & 1.8 & -1.6 & 0.2 & -0.6 & -0.8 & -0.5 & 0.1 & -0.4 & -0.1 \\
\hline Net foreign (exlc. project loans) & -3.3 & -1.0 & -1.6 & -1.5 & -0.8 & -0.7 & -0.5 & -0.6 & -0.7 \\
\hline Privatization & 0.1 & 0.1 & 0.1 & 0.4 & 0.3 & 0.3 & 0.0 & 0.0 & 0.0 \\
\hline Financing gap & 0.0 & 0.0 & 0.0 & 0.0 & 0.0 & 0.0 & 0.0 & 0.0 & 0.0 \\
\hline Local governments & 0.0 & 0.4 & 0.2 & 0.6 & 0.1 & 0.1 & 0.0 & 0.0 & 0.0 \\
\hline of which: privatization & $\ldots$ & $\ldots$ & $\ldots$ & 0.5 & $\ldots$ & 0.2 & $\ldots$ & 0.0 & $\ldots$ \\
\hline Social Fund & -0.2 & 0.0 & 0.2 & 0.1 & 0.3 & 0.2 & 0.0 & 0.0 & 0.0 \\
\hline Health Fund & -0.1 & -0.5 & 0.0 & -0.2 & 0.0 & -0.1 & 0.0 & 0.0 & 0.0 \\
\hline Project loans & 0.8 & 0.9 & 0.9 & 0.9 & 1.5 & 1.2 & 0.8 & 1.5 & 1.3 \\
\hline \multicolumn{10}{|l|}{ Memorandum item: } \\
\hline GDP (millions of lei) & 32,032 & 37,652 & 41,953 & 44,069 & 46,603 & 49,708 & 53,195 & 56,039 & 62,850 \\
\hline Augmented deficit 1/ & $\ldots$ & $\ldots$ & $\ldots$ & -0.3 & $\ldots$ & $\ldots$ & $\ldots$ & $\ldots$ & $\ldots$ \\
\hline
\end{tabular}

Sources: Moldovan authorities; and Fund staff estimates and projections.

1/ Includes lei $250 \mathrm{mln}$ of NBM recapitalization. 
Table 3a. Moldova: General Government Budget, 2006-07

(In millions of lei; unless otherwise indicated)

\begin{tabular}{|c|c|c|c|c|c|c|c|c|c|c|}
\hline & \multicolumn{2}{|c|}{2006} & \multicolumn{8}{|c|}{2007} \\
\hline & \multirow[b]{2}{*}{$\begin{array}{c}\text { IMF CR } \\
\text { No. 06/184 }\end{array}$} & \multirow[b]{2}{*}{ Prel. } & \multicolumn{2}{|c|}{ Q1 } & \multicolumn{2}{|c|}{ Q2 } & \multicolumn{2}{|c|}{ Q3 } & \multicolumn{2}{|c|}{ Q4 } \\
\hline & & & $\begin{array}{c}\text { IMF CR } \\
\text { No. } 07 / 45\end{array}$ & $\begin{array}{c}\text { Prel. } \\
\text { Actual }\end{array}$ & $\begin{array}{c}\text { IMF CR } \\
\text { No. } 07 / 45\end{array}$ & Proj. & $\begin{array}{c}\text { IMF CR } \\
\text { No. } 07 / 45\end{array}$ & Proj. & $\begin{array}{c}\text { IMF CR } \\
\text { No. 07/45 }\end{array}$ & Proj. \\
\hline Revenues and grants & 16,893 & 17,849 & 3,955 & 4,911 & 8,766 & 9,831 & 14,070 & 15,060 & 19,779 & 20,886 \\
\hline Revenues & 15,280 & 16,044 & 3,442 & 4,583 & 7,727 & 8,781 & 12,364 & 13,289 & 17,526 & 18,338 \\
\hline Tax revenues & 14,176 & 14,889 & 3,140 & 3,966 & 7,080 & 7,768 & 11,513 & 12,072 & 16,479 & 16,928 \\
\hline Profit tax & 975 & 1,079 & 292 & 550 & 511 & 732 & 715 & 897 & 989 & 1,143 \\
\hline Personal income tax & 1,051 & 1,128 & 225 & 295 & 502 & 585 & 805 & 905 & 1,124 & 1,243 \\
\hline VAT & 5,796 & 6,194 & 1,217 & 1,543 & 2,885 & 3,175 & 4,799 & 5,061 & 6,882 & 7,122 \\
\hline Excises & 1,004 & 1,071 & 212 & 260 & 506 & 544 & 854 & 881 & 1,228 & 1,246 \\
\hline Foreign trade taxes & 764 & 828 & 167 & 223 & 399 & 411 & 656 & 621 & 940 & 856 \\
\hline Other taxes & 336 & 342 & 54 & 53 & 156 & 149 & 273 & 258 & 395 & 373 \\
\hline Social Fund contributions & 3,723 & 3,691 & 798 & 866 & 1,753 & 1,813 & 2,862 & 2,916 & 4,154 & 4,204 \\
\hline Health Fund contributions & 526 & 558 & 176 & 176 & 368 & 360 & 549 & 533 & 768 & 743 \\
\hline Non-tax revenues & 1,104 & 1,154 & 302 & 617 & 648 & 1,013 & 851 & 1,217 & 1,047 & 1,410 \\
\hline NBM transfers & 163 & 163 & 0 & 313 & 118 & 313 & 118 & 313 & 118 & 313 \\
\hline Grants & 440 & 315 & 320 & 65 & 613 & 495 & 858 & 681 & 1,079 & 1,046 \\
\hline Budget support grants & 127 & 62 & 77 & 1 & 0 & 312 & 77 & 380 & 77 & 687 \\
\hline Foreign financed projects grants & 313 & 253 & 92 & 64 & 176 & 183 & 246 & 302 & 309 & 359 \\
\hline Other foreign grants & $\ldots$ & $\ldots$ & $\ldots$ & $\ldots$ & 346 & 0 & $\ldots$ & $\ldots$ & 693 & 0 \\
\hline Revenues of special funds & 1,174 & 1,490 & 193 & 264 & 426 & 555 & 848 & 1,090 & 1,174 & 1,501 \\
\hline Expenditures and net lending (cash) & 16,892 & 17,726 & 4,099 & 4,525 & 9,014 & 10,078 & 14,308 & 15,298 & 20,012 & 21,119 \\
\hline National Economy & 2,501 & 2,721 & 435 & 647 & 933 & 1,289 & 1,673 & 2,130 & 2,319 & 2,951 \\
\hline Social sphere & 5,076 & 5,496 & 1,205 & 1,385 & 2,514 & 2,800 & 3,779 & 4,194 & 5,353 & 5,880 \\
\hline Education & 3,357 & 3,605 & 859 & 866 & 1,798 & 1,763 & 2,627 & 2,711 & 3,625 & 3,936 \\
\hline Health care & 540 & 626 & 107 & 117 & 231 & 244 & 382 & 381 & 544 & 593 \\
\hline Other & 1,179 & 1,265 & 238 & 402 & 485 & 793 & 769 & 1,102 & 1,184 & 1,351 \\
\hline Interest payments & 537 & 455 & 152 & 141 & 300 & 282 & 487 & 453 & 676 & 645 \\
\hline Other expenditures & 2,792 & 3,249 & 736 & 819 & 1,735 & 1,771 & 2,802 & 2,893 & 3,895 & 4,130 \\
\hline Social Fund expenditures & 4,502 & 4,383 & 1,138 & 1,148 & 2,421 & 2,353 & 3,811 & 3,742 & 5,088 & 5,088 \\
\hline Health Fund expenditures & 1,528 & 1,485 & 443 & 403 & 925 & 909 & 1,378 & 1,400 & 2,012 & 1,938 \\
\hline Net lending & -43 & -63 & -9 & -18 & -14 & -27 & -22 & -48 & -24 & -63 \\
\hline Unidentified expenditure & $\ldots$ & $\ldots$ & $\ldots$ & 0 & $\ldots$ & 702 & $\ldots$ & 534 & $\ldots$ & 550 \\
\hline Statistical discrepancy & 0 & -5 & 0 & 0 & 0 & 0 & 0 & 0 & 0 & 0 \\
\hline Overall surplus(+)/deficit(-) (cash) & 0 & 118 & -144 & 386 & -248 & -248 & -238 & -238 & -233 & -233 \\
\hline Primary balance (cash) & 537 & 572 & 8 & 528 & 51 & 34 & 250 & 216 & 443 & 412 \\
\hline Overall surplus(+)/deficit(-) (commitments) & 30 & 131 & -144 & 286 & -248 & -248 & -238 & -238 & -233 & -233 \\
\hline Financing & 0 & -118 & 144 & -386 & 248 & 248 & 238 & 238 & 233 & 233 \\
\hline Budget financing & -388 & -528 & -40 & -463 & -71 & 4 & -234 & -128 & -470 & -346 \\
\hline Central government & -555 & -752 & -88 & -108 & -272 & 112 & -571 & -282 & -630 & -456 \\
\hline Net domestic & 71 & -279 & -154 & -42 & -203 & 163 & -457 & -186 & -390 & -268 \\
\hline Net foreign (exlc. project loans) & -653 & -641 & 67 & -77 & -109 & -102 & -194 & -187 & -395 & -343 \\
\hline Privatization & 28 & 168 & 0 & 12 & 40 & 52 & 80 & 92 & 155 & 155 \\
\hline Financing gap & 0 & 0 & 0 & -1 & 0 & -1 & 0 & 0 & 0 & 0 \\
\hline Local governments & 100 & 267 & -15 & -390 & -39 & -294 & -5 & -140 & 40 & 40 \\
\hline of which: privatization & $\ldots$ & 227 & $\ldots$ & 55 & $\ldots$ & 55 & $\ldots$ & 70 & $\ldots$ & 80 \\
\hline Social Fund & 67 & 30 & 113 & 109 & 203 & 199 & 298 & 294 & 120 & 120 \\
\hline Health Fund & 0 & -74 & -51 & -73 & 37 & -13 & 44 & 0 & 0 & -50 \\
\hline Project loans & 387 & 410 & 184 & 76 & 319 & 244 & 472 & 366 & 703 & 579 \\
\hline \multicolumn{11}{|l|}{ Memorandum item: } \\
\hline GDP (millions of lei) & 41,953 & 44,069 & $\ldots$ & $\ldots$ & $\ldots$ & $\ldots$ & $\ldots$ & $\ldots$ & 46,603 & 49,708 \\
\hline
\end{tabular}

Sources: Moldovan authorities; and Fund staff estimates and projections. 
Table 4. Moldova: Accounts of the National Bank of Moldova and Monetary Survey, 2005-07

(In millions of lei; unless otherwise indicated)

\begin{tabular}{|c|c|c|c|c|c|c|c|c|c|c|}
\hline & \multirow[t]{3}{*}{2005} & \multirow[t]{3}{*}{2006} & \multicolumn{8}{|c|}{2007} \\
\hline & & & \multicolumn{2}{|c|}{ Q1 } & \multicolumn{2}{|c|}{ Q2 } & \multicolumn{2}{|c|}{ Q3 } & \multicolumn{2}{|c|}{ Q4 } \\
\hline & & & $\begin{array}{c}\text { IMF CR } \\
\text { No. } 07 / 45\end{array}$ & Actual & $\begin{array}{c}\text { IMF CR } \\
\text { No. } 07 / 45\end{array}$ & Proj. & $\begin{array}{c}\text { IMF CR } \\
\text { No. } 07 / 45\end{array}$ & Proj. & $\begin{array}{c}\text { IMF CR } \\
\text { No. } 07 / 45\end{array}$ & Proj. \\
\hline \multicolumn{11}{|l|}{ National Bank of Moldova } \\
\hline Net foreign assets & 6,434 & 8,140 & 8,334 & 8,164 & 8,698 & 9,098 & 9,072 & 9,376 & 10,179 & 10,142 \\
\hline NFA (convertible) & 6,436 & 8,159 & 8,334 & 8,175 & 8,698 & 9,098 & 9,072 & 9,376 & 10,179 & 10,142 \\
\hline NFA (convertible) in program exchange rates & 6,436 & 8,260 & 7,737 & 8,500 & 7,967 & 9,483 & 8,207 & 9,830 & 8,789 & 10,705 \\
\hline Gross reserves & 7,666 & 10,005 & 9,782 & 9,843 & 10,611 & 11,110 & 10,871 & 11,264 & 12,303 & 12,210 \\
\hline Reserve liabilities & $-1,230$ & $-1,846$ & $-1,448$ & $-1,668$ & $-1,913$ & $-2,012$ & $-1,799$ & $-1,887$ & $-2,124$ & $-2,069$ \\
\hline Net domestic assets & 569 & $-1,628$ & $-1,594$ & $-1,667$ & $-1,812$ & $-2,084$ & $-2,039$ & $-2,153$ & $-2,924$ & $-2,698$ \\
\hline Net domestic assets in program exchange rates & 569 & $-1,728$ & -997 & $-2,003$ & $-1,081$ & $-2,468$ & $-1,174$ & $-2,606$ & $-1,535$ & $-3,261$ \\
\hline Net claims on general government & 2,291 & 1,683 & 1,607 & 1,556 & 1,516 & 1,744 & 1,205 & 1,367 & 565 & 545 \\
\hline Credit to banks & $-1,186$ & $-2,031$ & $-1,300$ & $-2,308$ & $-1,300$ & $-2,797$ & -950 & $-2,413$ & -600 & $-2,053$ \\
\hline Other items (net) & -536 & $-1,280$ & $-1,900$ & -915 & $-2,028$ & $-1,031$ & $-2,295$ & $-1,107$ & $-2,889$ & $-1,190$ \\
\hline Reserve money & 7,003 & 6,512 & 6,740 & 6,497 & 6,886 & 7,014 & 7,033 & 7,223 & 7,255 & 7,443 \\
\hline Currency in circulation & 4,571 & 5,146 & 5,190 & 4,923 & 5,302 & 5,401 & 5,416 & 5,562 & 5,586 & 5,731 \\
\hline Banks' reserves & 2,431 & 1,366 & 1,550 & 1,573 & 1,584 & 1,613 & 1,618 & 1,661 & 1,669 & 1,712 \\
\hline Required reserves & 795 & 936 & 903 & 958 & 936 & 1,009 & 963 & 1,057 & 983 & 1,137 \\
\hline Excess reserves & 1,635 & 430 & 648 & 615 & 648 & 604 & 655 & 604 & 685 & 575 \\
\hline \multicolumn{11}{|l|}{ Monetary Survey } \\
\hline Net foreign assets & 7,421 & 9,466 & 10,394 & 9,214 & 10,787 & 10,096 & 11,186 & 10,370 & 12,394 & 11,130 \\
\hline NFA (convertible) & 7,418 & 9,511 & 10,394 & 9,322 & 10,787 & 10,096 & 11,186 & 10,370 & 12,394 & 11,130 \\
\hline of which: commercial banks & 982 & 1,352 & 2,060 & 1,147 & 2,088 & 998 & 2,114 & 993 & 2,215 & 989 \\
\hline NFA (non-convertible) & 3 & -45 & 0 & -108 & 0 & 0 & 0 & 0 & 0 & 0 \\
\hline Net domestic assets & 8,405 & 10,092 & 9,199 & 10,734 & 9,360 & 11,319 & 9,495 & 11,661 & 9,282 & 11,990 \\
\hline Net claims on general government & 1,387 & 1,273 & 1,323 & 793 & 1,306 & 775 & 1,158 & 670 & 1,170 & 1,040 \\
\hline Credit to economy & 9,990 & 13,768 & 13,012 & 14,821 & 13,431 & 15,421 & 13,850 & 16,020 & 14,269 & 16,167 \\
\hline Other items (net) & $-2,972$ & $-4,949$ & $-5,136$ & $-4,880$ & $-5,377$ & $-4,877$ & $-5,513$ & $-5,030$ & $-6,156$ & $-5,217$ \\
\hline Broad Money (M3) & 15,827 & 19,558 & 19,594 & 19,948 & 20,147 & 21,415 & 20,682 & 22,031 & 21,676 & 23,120 \\
\hline Broad Money (M2: excluding FCD) & 11,126 & 12,485 & 12,133 & 12,716 & 12,502 & 13,808 & 12,820 & 14,373 & 13,148 & 15,208 \\
\hline Currency in circulation & 4,571 & 5,146 & 5,190 & 4,856 & 5,302 & 5,401 & 5,416 & 5,562 & 5,586 & 5,731 \\
\hline Total deposits & 11,256 & 14,412 & 14,404 & 15,092 & 14,844 & 16,014 & 15,266 & 16,469 & 16,090 & 17,389 \\
\hline Domestic currency deposits & 6,554 & 7,324 & 6,943 & 7,826 & 7,200 & 8,407 & 7,404 & 8,811 & 7,562 & 9,477 \\
\hline Foreign currency deposits (FCD) & 4,701 & 7,073 & 7,461 & 7,233 & 7,645 & 7,607 & 7,862 & 7,658 & 8,528 & 7,912 \\
\hline \multicolumn{11}{|l|}{ Memorandum items: } \\
\hline Reserve money growth (percent change; quarterly) & 20.3 & 5.0 & 1.9 & -0.2 & 2.2 & 8.0 & 2.1 & 3.0 & 3.1 & 3.0 \\
\hline Reserve money growth (percent change; annual) & 31.8 & -7.0 & 12.7 & 8.7 & 8.6 & 10.6 & 13.4 & 16.4 & 9.7 & 14.3 \\
\hline Broad money growth (percent change; quarterly) & 6.0 & 9.7 & 3.7 & 2.0 & 2.8 & 7.4 & 2.7 & 2.9 & 4.8 & 4.9 \\
\hline Broad money growth (percent change; annual) & 35.0 & 23.6 & 20.1 & 22.3 & 13.2 & 20.4 & 16.0 & 23.5 & 14.7 & 18.2 \\
\hline Credit to economy, (percent change, annual) & 35.0 & 37.8 & 20.9 & 37.7 & 16.6 & 33.9 & 12.4 & 30.0 & 13.3 & 17.4 \\
\hline Net credit to government, (percent change, annual) & -15.0 & -26.6 & -17.4 & -20.0 & -17.8 & -5.4 & -18.3 & -7.3 & -67.9 & -67.6 \\
\hline Gross international reserves (millions of U.S. dollars) & 597.4 & 775.3 & 683.3 & 786.1 & 731.2 & 896.0 & 740.0 & 912.6 & 799.3 & 994.2 \\
\hline Net international reserves (millions of U.S. dollars) & 501.6 & 632.2 & 582.1 & 652.9 & 599.4 & 733.7 & 617.5 & 759.7 & 661.3 & 825.8 \\
\hline Velocity (M3; end of period) & 2.4 & 2.3 & 2.2 & 2.3 & 2.2 & 2.2 & 2.2 & 2.26 & 2.15 & 2.15 \\
\hline Broad money multiplier & 2.3 & 3.0 & 2.9 & 3.1 & 2.9 & 3.1 & 2.9 & 3.0 & 3.0 & 3.1 \\
\hline Share of foreign currency deposits in all deposits & 41.8 & 49.1 & 51.8 & 47.9 & 51.5 & 47.5 & 51.5 & 46.5 & 53.0 & 45.5 \\
\hline
\end{tabular}

Sources: National Bank of Moldova; and Fund staff estimates and projections. 
Table 5. Moldova: Financial Sector Indicators, 2000-06

(End-of-period; in percent, unless otherwise indicated)

\begin{tabular}{|c|c|c|c|c|c|c|c|}
\hline & 2000 & 2001 & 2002 & 2003 & 2004 & 2005 & 2006 \\
\hline \multicolumn{8}{|l|}{ Size } \\
\hline Number of banks & 20 & 19 & 16 & 16 & 16 & 16 & 15 \\
\hline Total assets of the banking system (in percent of GDP) & 29 & 31 & 35 & 38 & 42 & 48 & 52 \\
\hline Credit to nongovernment sector (in percent of GDP) & 14 & 16 & 19 & 22 & 23 & 27 & 26 \\
\hline \multicolumn{8}{|l|}{ Capital adequacy } \\
\hline Capital adequacy ratio 1 / & 49 & 43 & 36 & 32 & 31 & 27 & 28 \\
\hline \multicolumn{8}{|l|}{ Liquidity } \\
\hline Liquidity ratio $2 /$ & 67 & 60 & 56 & 48 & 51 & 48 & 44 \\
\hline \multicolumn{8}{|l|}{ Asset quality 3/ } \\
\hline Nonperforming loans as a share of total loans & 21 & 10 & 8 & 6 & 7 & 5 & 4 \\
\hline Nonperforming loans, net of loan loss provisions / tier one capital & 15 & 5 & 3 & 2 & 3 & 0 & -3 \\
\hline Loans under supervision as a share of total loans & 30 & 31 & 35 & 35 & 34 & 39 & 41 \\
\hline Nonperforming and loans under supervision as a share of total loans & 51 & 41 & 43 & 42 & 41 & 44 & 45 \\
\hline \multicolumn{8}{|l|}{ Profitability } \\
\hline Return on equity & 25 & 14 & 17 & 20 & 18 & 15 & 21 \\
\hline Return on assets & 7 & 4 & 4 & 4 & 4 & 3 & 3 \\
\hline Non-interest income / Total income & 39 & 35 & 40 & 38 & 35 & 34 & 31 \\
\hline \multicolumn{8}{|l|}{ Interest rates } \\
\hline Domestic currency average lending rate & 32 & 26 & 19 & 19 & 21 & 17 & 19 \\
\hline Domestic currency average deposit rate & 24 & 18 & 13 & 14 & 16 & 11 & 13 \\
\hline Interest rate spread, domestic currency & 9 & 8 & 7 & 5 & 6 & 7 & 5 \\
\hline Foreign currency average lending rate & 15 & 12 & 12 & 11 & 11 & 11 & 11 \\
\hline Foreign currency average deposit rate & 5 & 3 & 3 & 4 & 5 & 5 & 6 \\
\hline Interest rate spread, foreign currency & 10 & 9 & 9 & 7 & 6 & 5 & 5 \\
\hline 91-day T-bill (effective nominal yield) & 25 & 8 & 11 & 18 & 7 & 4 & 12 \\
\hline \multicolumn{8}{|l|}{ Foreign currency deposits } \\
\hline Share of foreign currency deposits in broad money & 28 & 28 & 31 & 34 & 31 & 30 & 36 \\
\hline Share of foreign currency deposits in all deposits & 49 & 45 & 47 & 50 & 45 & 42 & 49 \\
\hline
\end{tabular}

Source: National Bank of Moldova.

1/ Total regulatory capital over total risk-weighted assets.

2/ Liquid assets over total deposits.

3/ Uncertainty regarding the ultimate beneficial owners of banks suggests caution is in order regarding the reliability of this data. 
Table 6: Moldova: Localized Millennium Development Goals (EGPRSP)

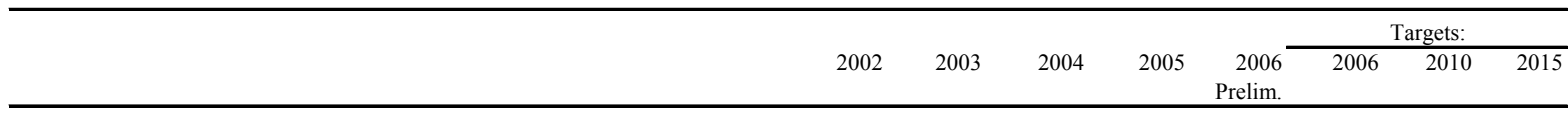

Goal: Eradicate extreme poverty and hunger

Target: Reduce by half the proportion of persons with daily income below 2.15 USD (PPP) over the period 1997-2015

- Population with income below $\$ 2.15$ (PPP) a day (percent)

Goal: Achieve universal access to secondary school education

Target: Ensure equal opportunities for all children to study in secondary schools

- Net enrollment ratio in secondary education (percent)

Goal: Reduce child mortality

Target: Reduce by two-thirds the under-five mortality rate over the period 1990-2015

- Under-five mortality rate (per 1,000 )

- Infant mortality rate (per 1,000 live births)

- Immunization, measles (percent of children under 2 years old)

Goal: Improve maternal health

Target: Reduce the maternal mortality ratio by three-fourths over the period 1990-2015

- Maternal mortality ratio (per 100,000 births)

- Births attended by skilled health personnel (percent)

Goal: Combat HIV/AIDS, tuberculosis and malaria

Target: Have halted by 2015 , and begun to reverse, the spread of HIV/AIDS to improved water sources
- HIV/AIDS incidence (per 100,000 people)

- HIV incidence among 15-24 year-olds

Target: Have halted by 2015, and begun to reverse, the incidence of tuberculosis and malaria

- Mortality rate associated with tuberculosis (deaths per 100,000 people)

Goal: Ensure environmental sustainability

Target: Integrate the principles of sustainable development into country policies and programs and reverse the loss of environmental resources

- Proportion of land areas covered by forest (percent)

- Ratio of area protected to maintain biological diversity (percent)

11.

11.4
2.0

$$
\begin{array}{r}
12.4 \\
2.0
\end{array}
$$$$
12.6
$$$$
2.0
$$$$
2.0
$$$$
4.7
$$

Target: Halve, by 2015, the proportion of people without sustainable access

- Population with sustainable access to an improved water source (percent)

Target: Achieve a significant increase of the share of people with access to improved sanitation

- Population with access to improved sanitation (percent)

$41.7 \quad 41.5 \quad 43.6$

$\begin{array}{llll}10.0 & 4.0 & 3.5 & 3.2\end{array}$

$\begin{array}{llll}13.3 & 4.9 & 4.2 & 4.0\end{array}$

$\begin{array}{lllllllll}39.5 & 39.7 & 44.5 & \ldots & \ldots & 47.7 & 57.0 & 68.5\end{array}$

Sources: Economic Growth and Poverty Reduction Strategy Paper (EGPRSP) 2004-06, EGPRSP Monitoring Unit 
Table 7. Moldova: External Financing Requirements and Sources, 2004-09 (In millions of U.S. dollars)

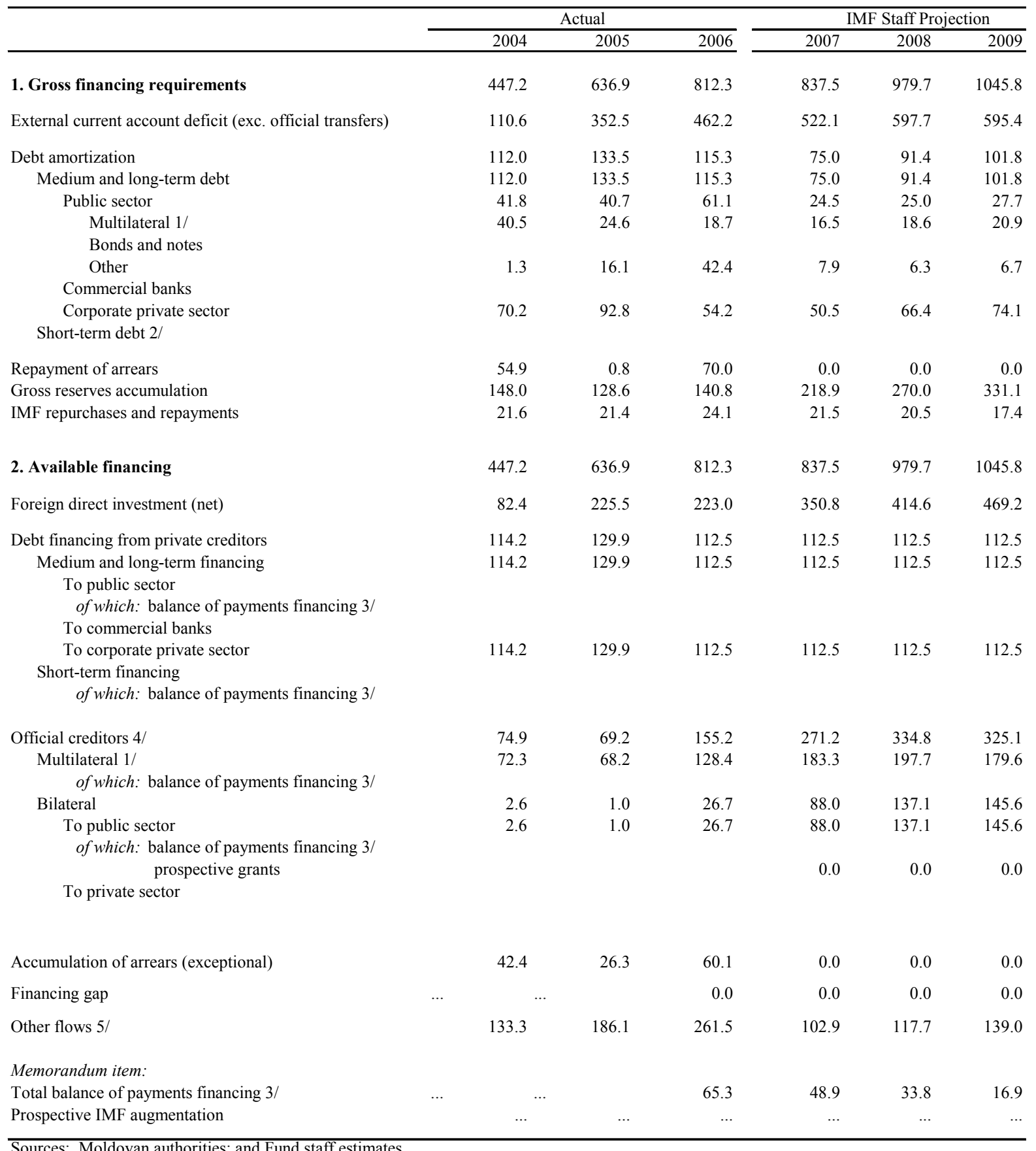

Sources: Moldovan authorities; and Fund staff estimates.

1/ Excluding the IMF.

2/ Original maturity of less than 1 year. Stock at the end of the previous period.

3/ Includes those transactions that are undertaken for the purpose of financing a balance of payments deficit or an increase in reserves.

4/ Includes both loans and grants.

5/ Includes all other net financial flows, and errors and omissions. 


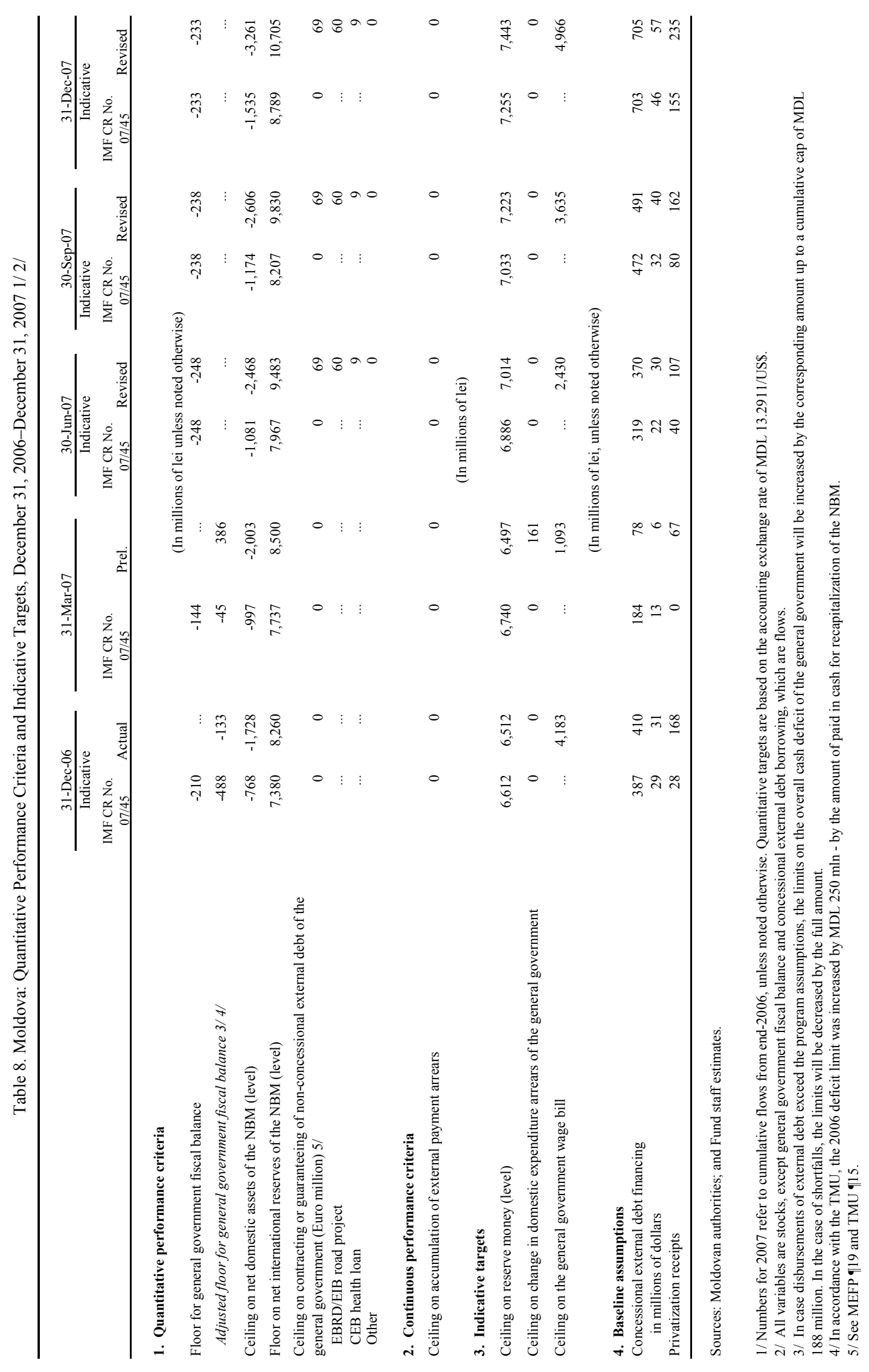




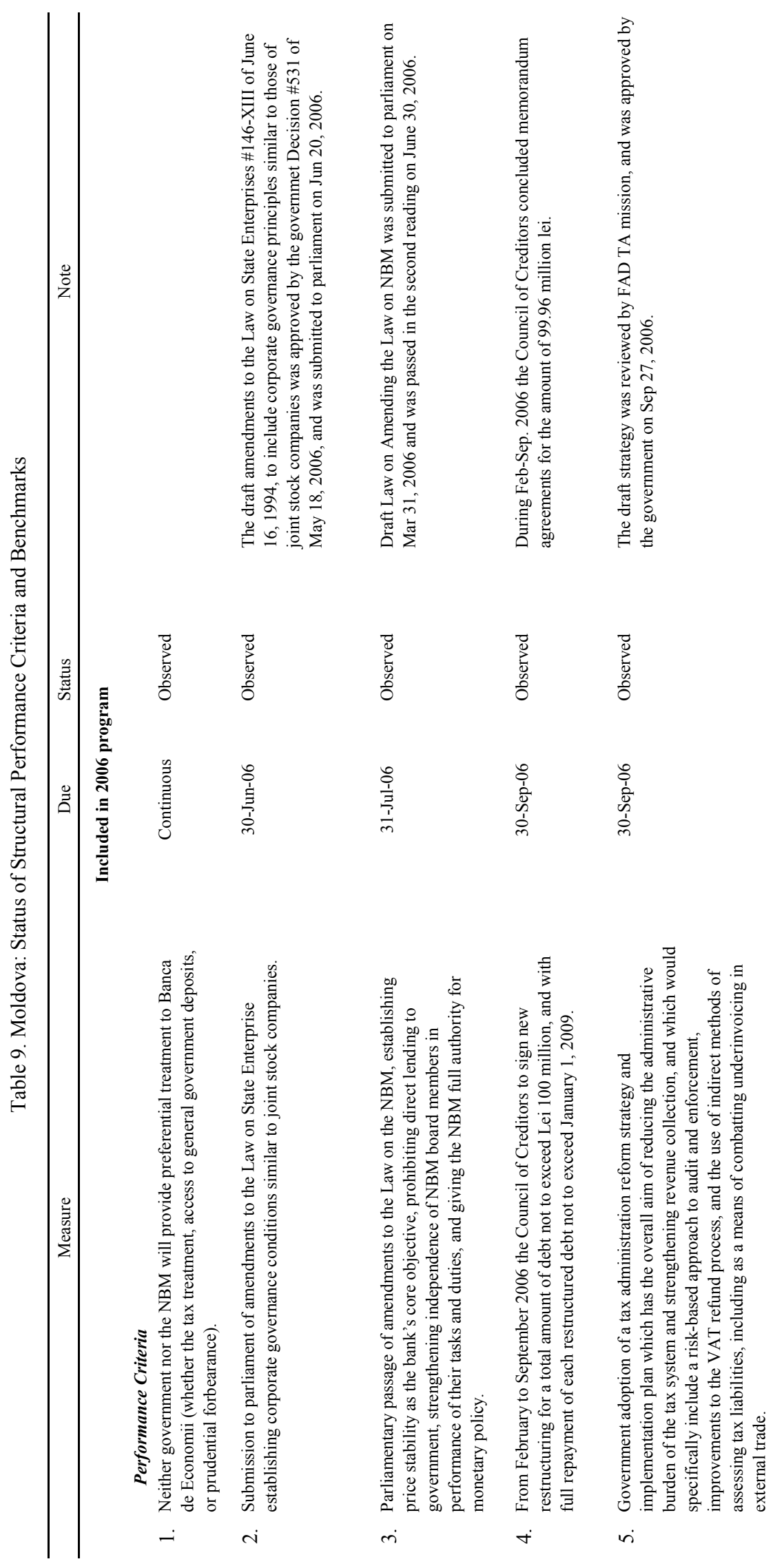




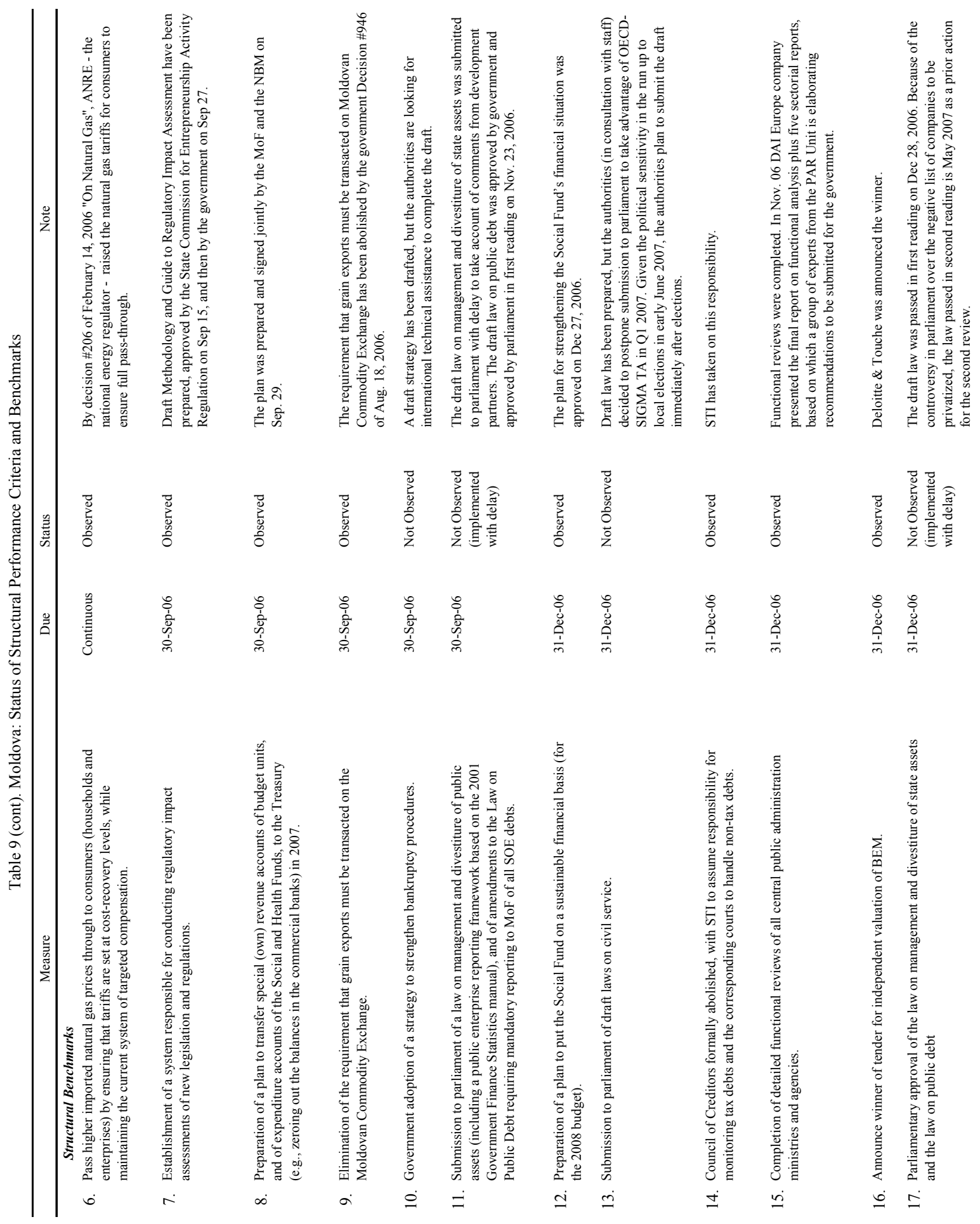




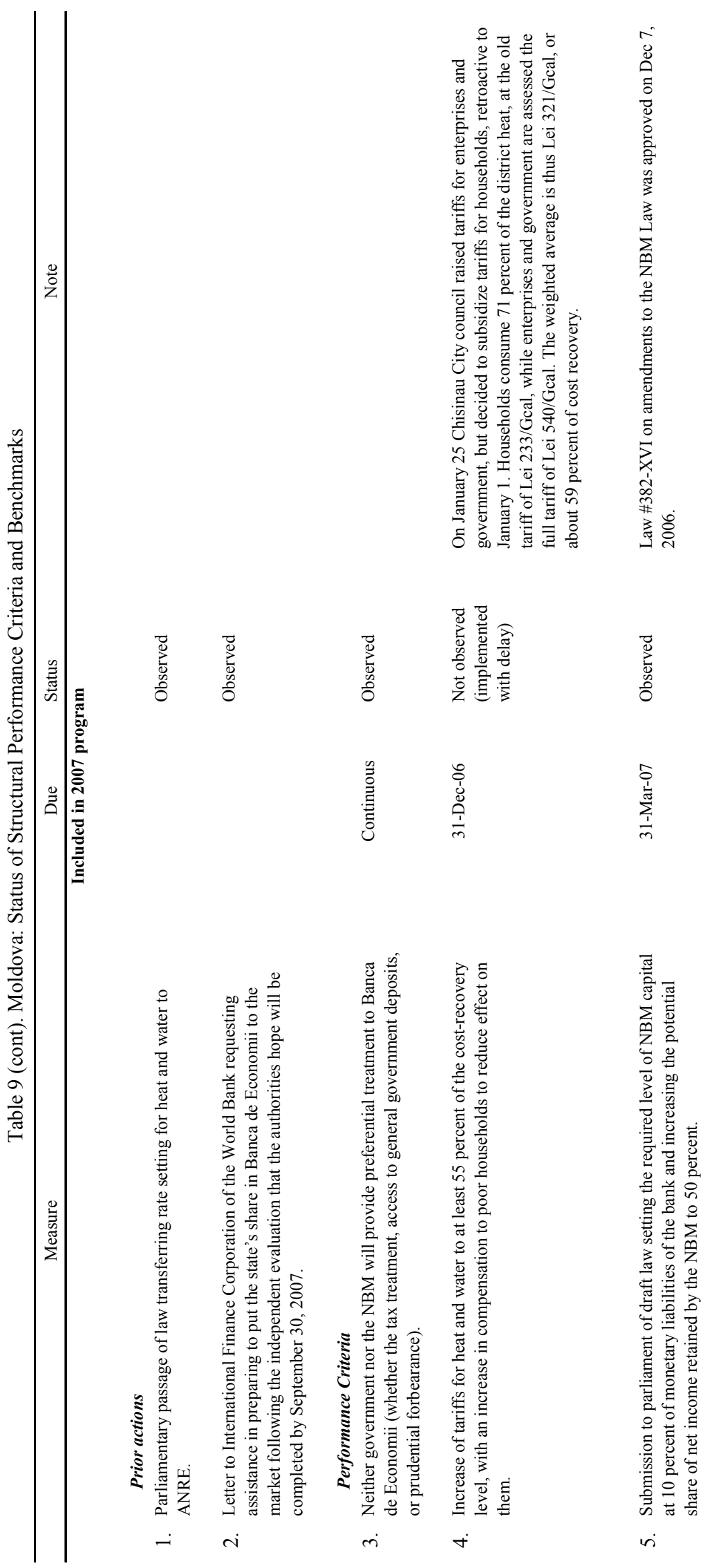




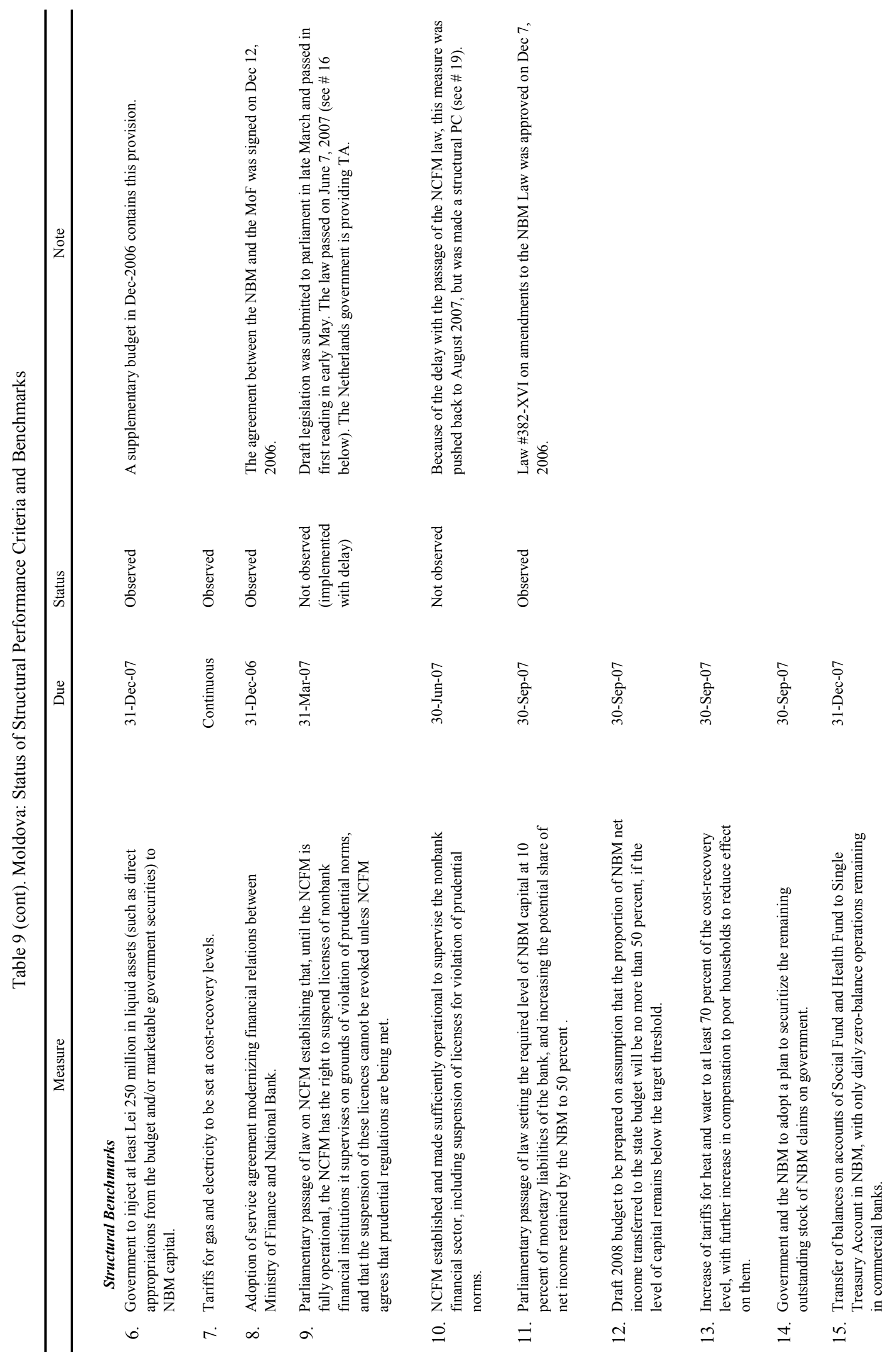




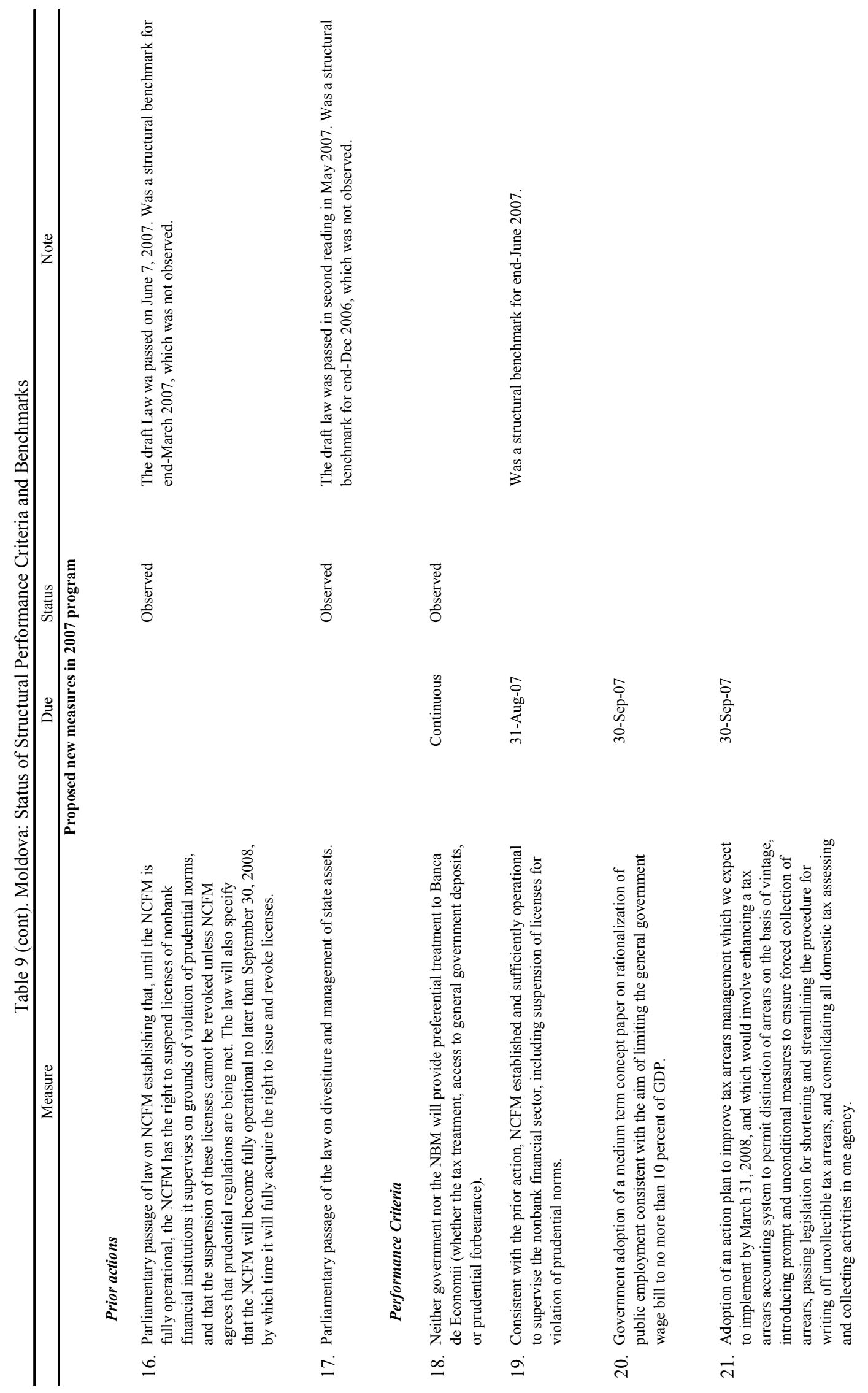




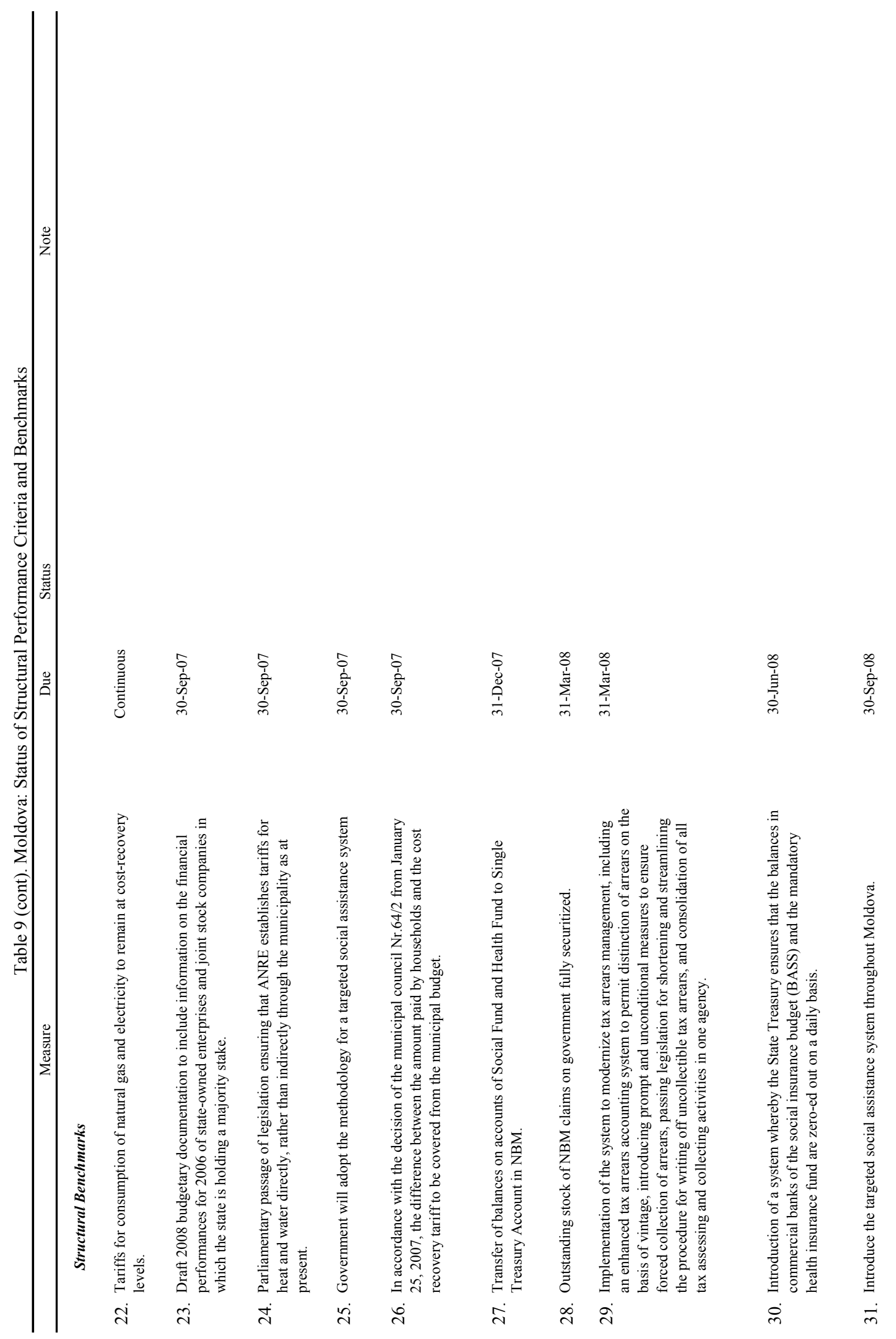




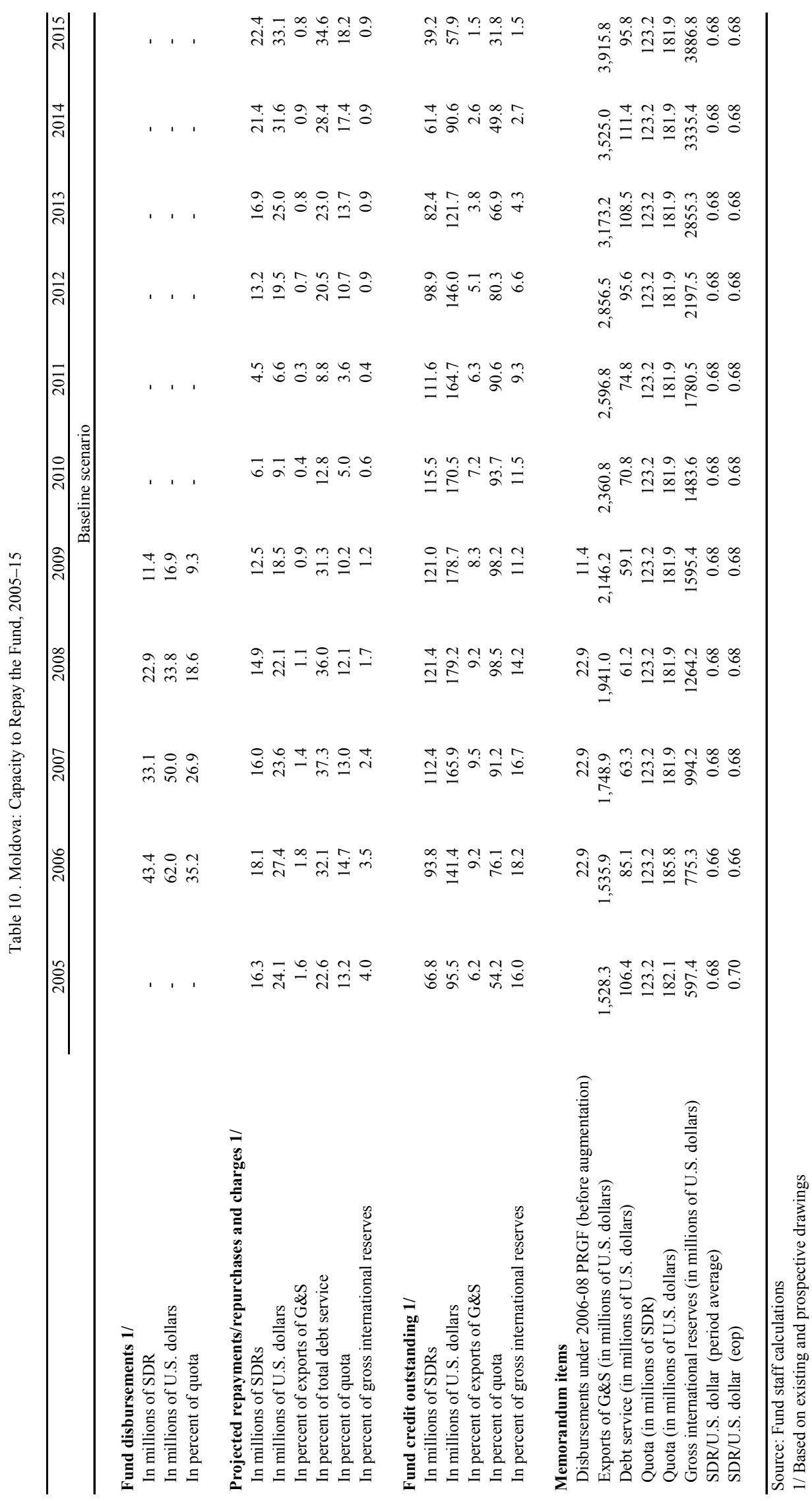


Table 11. Moldova: Reviews and Disbursements Under the Three-Year PRGF Arrangement

\begin{tabular}{lll}
\hline Date & Action & Disbursement \\
\hline May 5, 2006 & Board approval of the three-year arrangement & SDR 11.44 million \\
December 15, 2006 & $\begin{array}{l}\text { Complete first review based on end-September 2006 performance } \\
\text { criteria }\end{array}$ & SDR 31.97 million \\
On or after June 10, 2007 & $\begin{array}{l}\text { Complete second review based on end-March 2007 performance } \\
\text { criteria, and adopt conditions and disbursements for the second year of } \\
\text { the arrangement }\end{array}$ & SDR 21.71 million \\
On or after December 10,2007 & $\begin{array}{l}\text { Complete third review based on end-September 2007 performance } \\
\text { criteria }\end{array}$ & SDR 11.44 million \\
On or after June 10, 2008 & $\begin{array}{l}\text { Complete fourth review based on end-March 2008 performance } \\
\text { criteria, and adopt conditions and disbursements for the third year of } \\
\text { the arrangement }\end{array}$ & SDR 11.44 million \\
On or after December 10, 2008 & $\begin{array}{l}\text { Complete fifth review based on end-September 2008 performance } \\
\text { criteria }\end{array}$ & Complete sixth review based on end-March 2009 performance criteria \\
On or before May 4, 2009 & SDR 11.44 million \\
\hline
\end{tabular}




\section{AtTAChMent I}

June 25, 2007

Mr. Rodrigo de Rato

Managing Director

International Monetary Fund

$70019^{\text {th }}$ Street NW

Washington, DC 20431 USA

Dear Mr. de Rato:

The attached Memorandum is an update to the previous version of the Memorandum of Economic and Financial Policies(MEFP) for 2007. It describes policies and measures we intend to implement this year and for which we request the support of the International Monetary Fund under the three-year Poverty Reduction and Growth Facility (PRGF). These policies are consistent with our Economic Growth and Poverty Reduction Strategy (EGPRSP) as well as with the action plan agreed between the European Union and the Republic of Moldova.

Now that the external shocks have receded, the government and the National Bank believe that all efforts should focus on the core goals of the program, which are to promote sustainable growth and reduce poverty. To this end, the policies set forth in the attached memorandum aim at ensuring macroeconomic stability and financial sector development, and improving the business environment, including through reduction of the footprint of the state in the economy. In addition, in consultation with the Fund, we will take additional measures that may become appropriate for reaching these objectives.

We hereby request completion of the second review under the PRGF arrangement, and we also request a waiver for nonobservance of the performance criterion on raising heating tariffs in Chişinău to 55 percent of cost-recovery at end-December 2006. This nonobservance was technical as tariffs were increased on January 25 with the retroactive effect to January 1. Tariffs for water were raised as of February 1, though the financial impact of this delay was small. We also regret that an indicative ceiling on non-accumulation of domestic arrears was breached at end-March 2007 as the Chişinău city council delayed payment of the subsidy to compensate households for the increased heating costs. This nonpayment was also technical in nature as the decision to subsidize households came in when the municipal budget had already been long approved and thus did not contain the necessary 
allocation. The problem will be corrected at the first opportunity when the municipal budget is revised during the summer.

We will communicate to the IMF the information needed to monitor progress in implementing the program, and will conduct discussions with the Fund for the third review under the PRGF arrangement before end-December 2007.

We are committed to transparency, and thus we authorize the IMF to disseminate the MEFP and the associated Technical Memorandum of Understanding, as well as staff report that will be examined by the IMF Executive Board.

Sincerely yours

\author{
$\frac{/ \mathrm{s} /}{\text { Vasile Tarlev }}$ \\ Prime Minister \\ Government of the Republic of Moldova
}

$\frac{/ \mathrm{s} /}{\text { Mihail Pop }}$
Minister
Ministry of Finance

$\frac{/ \mathrm{s} /}{\text { Leonid Talmaci }}$
Governor
National Bank of Moldova

Attachments: Updated Memorandum of Economic and Financial Policies for 2007 Technical Memorandum of Understanding 


\title{
Attachment II: Updated Memorandum of Economic and Financial Policies for 2007
}

\author{
25 June 2007
}

\section{A. Introduction}

1. The present Memorandum is an updated version of the Memorandum of Economic and Financial Policies for 2007, and it summarizes our current view on the strategic objectives and priorities of the Republic of Moldova for the current year. Now that we have passed through the most difficult period of adjusting to the external shocks - including with the help of the international community, which supported our reform efforts at a Consultative Group meeting in Brussels in December-our central objective for 2007 is to ensure that the key goals and priorities of the three-year PRGF-supported program are achieved.

2. Economic performance in 2006 was influenced by a number of external factors, in particular the disruption of exports to the Russian Federation and a doubling in the price of energy imports. At 4 percent, real economic growth was weaker than in recent years. The trade deficit rose by 36.7 percent as import growth was strong and exports fell because of the Russian ban on imports of Moldovan wine, as well as difficulties in penetrating new export markets. Similarly, the 14.1 percent inflation outcome for 2006, significantly higher than in 2005, largely stemmed from accommodating higher prices of imported energy resources.

3. The economic outlook has improved recently, as some progress has been achieved in restoring economic relations with the Russian Federation. In particular, we have secured a medium-term agreement for the supply of natural gas, and settled on a framework to facilitate the resumption of wine exports. Although exports to the Russian Federation decreased by 47.6 percent in 2006, some progress was registered in diversifying exports, with sales to Central and Eastern Europe rising by 43.1 percent and by 13.6 percent to the countries of the European Union as well as to some counties of the CIS. As a result of the efforts of the National Bank of Moldova (NBM) in sterilizing excessive liquidity in the market, supported by limiting the fiscal deficit to lei 126.1 million ( 0.3 percent of GDP) in 2006, inflation fell to 11.2 percent by March 2007, and cumulative inflation during the first quarter of 2007 was 2.1 percent compared with 4.8 percent during the same period of the previous year.

\section{B. Program Performance}

4. With a few exceptions, the government and the NBM have implemented the actions included in the November 29, 2006, Memorandum on Economic and Financial Policies (MEFP). In particular, by March 31, 2007:

a. The parliament of the Republic of Moldova approved a law according to which local public authorities, including the Municipal Council of the City of Chisinau, are obliged to set tariffs for communal services on supply of heat and drinking water in 
line with the methodology approved by the National Energy Regulatory Agency (ANRE).

b. In line with the new law, the Municipal Council of the City of Chisinau approved new tariffs for communal services on the supply of heating equal to 100 percent of the cost recovery. Given that the development of a targeted social assistance system has been delayed, the municipal budget is temporarily covering the cost of the increase for households; tariffs for enterprises and budget sector institutions are not compensated. Thus, the weighted average tariff at the end of 2006 was 59.5 percent, exceeding the 55 percent threshold identified in the program. The tariffs for supply of drinking water remain differentiated for households and enterprises, with the weighted average tariff constituting almost 70 percent of cost recovery. The municipality will compensate the increase of tariff for the supply of drinking water only to some vulnerable categories of population (such as pensioners).

c. The ministry of finance is actively seeking the assistance of the International Finance Corporation in preparing Banca de Economii (BEM) for privatization, the timetable for which shall be determined upon completion of the evaluation to be conducted in 2007. Moreover, a winner of the tender to conduct this evaluation was announced on 29 December 2006.

d. The ministry of social protection, family, and children and the National Social Insurance House - in coordination with the ministry of finance, ministry of economy and trade and Main State Tax Inspectorate-have approved an action plan meant to ensure medium-term financial stability of the state social insurance budget.

e. Pursuant to the law No. 223-XVI dated July 14, 2006 amending the law of insolvency, the Council of Creditors ceased its activity on September 30, 2006 and was subsequently dissolved on December 31, 2006. Thereafter, the responsibility for monitoring tax and non-tax liabilities was entrusted to the State Tax Inspectorate as well as to the courts.

f. By the end of 2006, the second - "vertical" - stage of the functional analysis of subordinated institutions and some deconcentrated services was conducted under the central public administration reform. This analysis covered the lower level units of central agencies that had been analyzed during the first stage of the reform, as well as some that had not been covered by that analysis.

g. Financial relations between the National Bank of Moldova and the ministry of finance were upgraded by the signing on December 12, 2006 of an agreement on payment for rendering services to the ministry of finance by the National Bank of Moldova, and on the payment of market-related interest rates on government deposits held in the Treasury account in the NBM.

h. On December 7, 2006, the parliament of the Republic of Moldova approved the law No. 382-XVI amending the law of the National Bank of Moldova to stipulate that the 
capital of the NBM will grow dynamically with the size of the bank's balance sheet, either through the retention of profit or through contributions made by the government of the Republic of Moldova. In particular, the capital of the NBM should equal 10 percent of the monetary liabilities of the NBM (except for the liabilities to the government of the Republic of Moldova and to the International Monetary Fund), while up to 50 percent of the NBM's net profit can be retained by the NBM. To ensure that the target level of capital was reached at end-2006, the government injected lei 250 million to the charter capital of the National Bank of Moldova, directly from the state budget.

i. A supervisory board was established on April 2, 2007 to oversee our tax administration reform strategy. The board comprises representatives of the Main State Tax Inspectorate, ministry of finance, ministry of economy and trade, ministry of informational development, government office and representatives of professional and public associations. At the same time, the State Tax Inspectorate has set up a working group to manage the reform and coordinate activities with foreign donors.

j. In December 2006, the parliament approved the new law on public debt, state guarantees and state on-lending.

Implementation of three program measures was delayed, relative to the program timetable. These measures have now been or will shortly be completed.

- $\quad$ On May 4, 2007, the parliament of the Republic of Moldova adopted a law on denationalization and management of public property. This law is intended to reinvigorate the privatization process and improve management of public property. In particular, the new law includes a negative list of assets, which for now are not subject to privatization; all other state assets can be sold. In addition, on the basis of this law the government has produced a list of regulatory acts to be drafted and implemented in the field of managing state assets.

- $\quad$ The draft law on public functions and the statute of civil servants will shortly be submitted to parliament by June 11. Submission of the draft law was delayed to permit incorporation of the recommendations of experts from OECD-SIGMA. It envisages (i) establishing a central administrative body responsible for managing personnel recruited for civil service; (ii) recruiting and promoting in civil service on the basis of merit; and (iii) separating political from administrative functions.

- $\quad$ On June 7, 2007, the parliament of the Republic of Moldova adopted a set of legislative acts creating the National Commission for the Financial Markets (NCFM), which will combine functions currently exercised by the authorities supervising the securities market, insurance market and nongovernmental pension funds, savings and loan associations, credit history bureaus, and mortgage companies. The competencies of the newly created supervisory authority for the nonbank financial sector will not include the banking system, which will continue to be supervised by the National Bank of Moldova. Initially, the law gives the NCFM the right to suspend licenses 
held by non-bank financial institutions and stipulates that the validity of licenses cannot be restored without the consent of the NCFM. By the end of September 2008, the NCFM shall become the sole authority vested with the right to issue and revoke licenses for enterprises rendering financial non-banking services.

\section{Program Objectives}

5. Despite the persistent effects of the external shocks encountered last year, the key objectives pursued by the government for 2007 remained unchanged. Our goal is to reduce poverty by ensuring macroeconomic stability and sustainable economic growth, and we hope to achieve this by limiting government involvement in the economy, developing the financial sector, creating a favorable investment environment, stimulating the development of small and medium-sized business, rehabilitating infrastructure, promoting exports, creating new jobs in the country and granting social protection to vulnerable categories of population. These objectives, which include achieving the Millennium Development Goals, are clearly stated in the Economic Growth and Poverty Reduction Strategy Paper (EGPRSP), implementation of which shall continue through 2007, and in the EU-Republic of Moldova Action Plan.

6. While the EGPRSP will extend through 2007, beyond that the objectives of the government will be outlined in the National Development Plan for 2008-11 (NDP), which is now in preparation. The government's intention is to focus on the short list of strategic priorities, both economic and political. In the draft NDP that will be discussed within the participatory process, the five fundamental priorities are as follows:

- Consolidating a modern democratic state based on the principle of the rule of law;

- Settling the Transnistrian conflict and promoting reintegration of the country;

- Enhancing the competitiveness of the national economy;

- $\quad$ Developing human resources, promoting employment and facilitating social inclusion; and

- $\quad$ Promoting regional development.

One of the objectives of the NDP is the integration of key external commitments of the Republic of Moldova into one basic strategy (without replacing agreed bilateral policy documents) and establishing a single system of monitoring implementation of these commitments.

\section{Fiscal Policy}

7. Fiscal policy for 2007 shall remain tight, given continued high inflation and the need to ensure macroeconomic stability and to foster confidence of the population in the program more broadly. In this context, the government will maintain the budget deficit at 0.5 percent of GDP. 
8. For many years, budget sector wages have been insufficient to attract and retain qualified employees, many of whom have opted instead to work abroad. Thus, wages in the budget sector are to be increased, on average, by 12 percent in 2007 and by 23 percent in 2008 , following a significant (45 percent) increase in 2006. To finance the increase, we will set the size of the general government wage bill at about 10 percent of GDP. Further, the government intends to begin a medium-term program of public employment rationalization. By end-September 2007, we will prepare a multi-year concept paper, the goal of which is to attract and retain the best performing and most promising staff. The concept paper will include plans for a census of budget sector workers, an inventory of their wage and nonwage remuneration, and an assessment of sectoral needs for highly qualified staff. Although we will carry it out on our own if necessary, we hope to secure the support of international development partners in this work, both on an analytical level and - if possible - in terms of financial support to facilitate the reform.

9. To promote investment, both foreign and domestic, we have announced a major reform of corporate income taxation, as well as a wide-ranging amnesty of tax arrears and a liberalization of the rules governing legalization of capital. Corporate income tax rates will be set to zero, except when dividends are paid and non-business expenses are incurred, and all pre-2007 tax arrears (and associated fines and penalties not paid at the time the law goes into effect) will be cancelled. We understand that these reforms entail some risks, particularly to budget revenue, so we are prepared if necessary to take compensating measures to ensure that the fiscal and macroeconomic targets in the program will be achieved. With the aim of ensuring that the tax amnesty occurs only once, we intend to accelerate implementation of our strategy to strengthen tax administration. Thus, by end-September 2007, we will adopt an action plan to improve the system of tax arrears management, which would involve enhancing the tax arrears accounting system to permit distinction of arrears on the basis of vintage, introducing prompt and unconditional measures to ensure forced collection of arrears, passing legislation for shortening and streamlining the procedure for writing off uncollectible tax arrears, and consolidating all domestic tax assessing and collecting activities in one agency. We expect this plan to be implemented by March 31, 2008. Finally, we recognize that the capital legalization could pose some risk for our anti-money laundering (AML) regime, so we intend to accelerate the adoption of amendments to the AML law (prepared with assistance from the IMF and other partners), and will discuss the implications of the capital legalization with MONEYVAL and key bilateral partners.

10. The government continues to view reducing Moldova's vulnerability to international energy price changes as a key priority. Therefore, the authorities will continue to ensure that tariffs for natural gas and electricity remain at full cost recovery for all categories of consumer. Further, we will gradually move to increase tariffs for heat and water to full cost recovery, while also improving the targeting of social assistance to ensure that poor households do not suffer unduly in the adjustment process. Specifically, we will adopt the methodology for our targeted social assistance system by September 30, 2007, and introduce it throughout Moldova by September 30, 2008. Insofar as enterprises and public sector institutions currently pay a full cost-recovery tariff, the tariff paid by households will be increased sufficiently to reach a weighted average tariff paid by all categories of consumer of heat in Chişinău equal to 70 percent of cost recovery by September 30, 2007. In accordance 
with the decision of the municipal council Nr.64/2 from January 25, 2007, the difference between the amount paid by households and the cost recovery tariff will be covered from the municipal budget. Finally, we will amend the law to ensure that the regulatory authority (ANRE) establishes tariffs for heat and water directly, rather than indirectly through the municipality as at present.

\section{E. Monetary and Financial Policies}

11. During the balance of 2007, the National Bank of Moldova will continue to maintain a tight and prudent monetary policy with the aim of achieving a single-digit level of inflation. The program envisages that the monetary base will increase by 14.3 percent over the year to end-December, while interest rates will remain positive in real terms. This objective will be achieved by applying indirect instruments of monetary policy, and will be facilitated by the strengthened capital position of the NBM, as well as by the transfer of remaining deposits of the health and social funds to the single treasury account in the NBM.

12. The priority objective of our monetary policy is to ensure price stability. Thus, the National Bank of Moldova will continue to maintain a floating exchange rate and will intervene on the foreign exchange market mainly to smooth out volatility in the exchange rate, which we expect will permit an increase in international reserves to a level equivalent to almost three months of prospective imports.

13. We are considering the possibility of moving to an inflation targeting (IT) framework at an appropriate point in the future. Although we realize that Moldova is not yet prepared to move to inflation targeting, we are taking a number of intermediate steps to improve the monetary policy framework, and thus to lay the groundwork for IT in the future. In this context, when carrying out sterilization of excessive liquidity, the NBM will announce either a maximum interest rate, or the volume of intended sales. Further, beginning with the 2008 budget, the law on the budget will not oblige the NBM to roll over treasury bills; instead, coordination of monetary and fiscal policy will be handled by the joint NBM-Ministry of Finance liquidity managing committee. Further, in order to ensure that the NBM will have the tools to conduce monetary policy, by September 30, 2007, the ministry of finance and the NBM will adopt a plan for securitizing the stock of government debt owed to the NBM, with the securitization to take place in the first quarter of 2008.

14. After the legislation to establish the National Commission for the Financial Markets (NCFM) is passed, the expectation is that it will begin operations by August 31, 2007, including having the right to suspend the licenses of nonbank institutions in violation of prudential norms (and this suspension may not be revoked without the NCFM's approval). The legislation specifies that the NCFM will be financially and operationally independent, and that, once it reaches its full operational capacity - but not later than September 30, 2008 - it will fully acquire the right to issue and revoke licenses for all types of supervised non-banking financial activity.

15. We are committed to privatize BEM to a strategic banking investor following completion of the market valuation to be completed by year-end, and possibly by September 
30, 2007. Further, we have solicited the assistance of the International Financial Corporation in preparing to bring BEM to market shortly after completion of evaluation. In the meantime, the government and the NBM will continue to abstain from granting preferential treatment to the bank, including as regards taxation, prudential regulation or access to resources.

16. We have solicited an update under the Financial Sector Assessment Program (FSAP) in order to assess our progress in improving prudential regulation, transparency in ownership of the banking sector, and supervision of the nonbank financial sector. It is hoped the FSAP update will take place in the second half of 2007.

\section{F. Structural Reforms}

17. The external shocks faced by Moldova in 2006 highlighted the need to accelerate structural reforms to facilitate resumption of rapid economic growth. As a consequence, during the balance of 2007 and over the medium term, we intend to take significant measures to make the public sector more efficient and to improve the business environment. We will pay special attention to measures aimed at promoting exports and diversifying export markets.

\section{Trade policy and promotion of investments}

18. The government is implementing its strategy for attracting investment and promoting exports during 2006-15. During 2007, the key priorities include (i) obtaining autonomous trade preferences from the EU; (ii) conducting a detailed study of the economic implications for Moldova stemming from Romania's accession to the EU; (iii) creating in 2008 accredited laboratories for testing and ensuring quality of a number of Moldovan products (starting with wine and expanding it onto other farm products) in compliance with the European standards; (iv) implementing reform on granting entrepreneurial permits; and (v) establishing simplified methods of accounting for small and medium-sized enterprises.

19. The government has also prepared a concept paper on public-private partnerships (PPP), which will offer a wide spectrum of instruments and mechanisms for cooperation and interaction between the public and private sectors to increase infrastructure investment. The concept paper envisages that contingent liabilities that could appear in this process will be transparently reported to the financial market as well as to the parliament. In this connection, once the law is passed, we intend to consult with the staff of the IMF before concluding any significant PPP agreements. In this context, we will also consult with the staff of the IMF prior to entering into the second phases of the EBRD loan agreement and the EIB loan agreement for the road rehabilitation project (in the amount of $€ 17.5$ million each) to ensure that grant financing is obtained sufficient to ensure that the level of concessionality on these phases reaches the target of at least 35 percent.

20. Development of small and medium-sized business is one of the key elements that will contribute to economic growth and poverty reduction in Moldova. Thus, during 2007 we will implement our Strategy for developing small and medium-sized business, as well as the law of granting support to small and medium-sized enterprises. 


\section{Public administration reform}

21. We have completed a functional analysis of all levels of central public administration, and based on these results and recommendations, during 2007-08 we plan to i) approve the finalized mandate of these institutions, and ii) amend their regulations with the aim of reassigning their tasks and responsibilities. A policy analysis and coordination unit will be set up to coordinate policies within the government office and, based on an evaluation of experience gained by the pilot units created in seven ministries, policy analysis, monitoring and evaluation units might be set up in the remaining ministries. Finally, the government intends to create a unit at the central level authorized to improve human resource management policies and procedures in the civil service.

\section{Regulatory reform}

22. The second stage of the regulatory reform will continue in 2007, including revising the legislative framework in compliance with the law on basic principles and mechanisms of regulating entrepreneurial activity. The government will implement the National Strategy of Regulatory Reform, including by regularizing the system of granting entrepreneurial permits. Similarly, elements of the "one-stop shop" approach will be introduced into the activity of public authorities, including through the electronic exchange of data. The regulatory impact assessment (RIA) methodology will be introduced as well to limit excessive state intervention in the economy.

23. The government will continue efforts to improve and facilitate insolvency and bankruptcy procedures. In this context, we intend to adopt measures to simplify and reduce the time necessary to liquidate a business, including once again the "one-stop shop" principle.

\section{Public finance management}

24. The government will further improve public finance management by reforming budgetary institutions and aligning the legislative framework with EU requirements and standards. During 2007, the ministry of finance jointly with the National Social Insurance House and the National Health Insurance Company will develop (and by June 30, 2008 implement) a system to manage the state social insurance budget (BASS) as well as that of the mandatory health insurance fund through the State Treasury, which shall ensure zero balance on the accounts of the commercial banks at the close of every business day. While working out the state budget for 2008 the government intends to continue eliminating special funds.

25. Simultaneously with preparing the 2008 draft budget law the government, as part of budgetary documentation, will submit information on the financial performances for 2006 of state-owned enterprises and joint stock companies in which the state is holding a majority stake. 
Table 1. Prior Actions, Performance Criteria and Benchmarks ${ }^{3}$

\section{Prior Actions}

Parliamentary passage of law on NCFM establishing that, until the NCFM is fully operational, the NCFM has the right to suspend licenses of nonbank financial institutions it supervises on grounds of violation of prudential norms, and that the suspension of these licenses cannot be revoked unless NCFM agrees that prudential regulations are being met. The law will also specify that the NCFM will become fully operational no later than September 30,2008, by which time it will fully acquire the right to issue and revoke licenses. ( $₫ 14)$

Parliamentary passage of the law on divestiture and management of state assets. ( $(4)$

\section{Structural Performance Criteria}

\section{Continuous}

Neither the government nor the NBM will provide preferential treatment to Banca de Economii (including tax treatment, prudential regulation and access to resources). ( $\mathbb{1} 15$ )

\section{August 31, 2007}

As a follow-up to the measures taken under the prior action, implementation of all actions necessary to establish the NCFM, including the appointment of a chairman, and to grant the NCFM the operational ability to suspend licenses of nonbank financial institutions in violation of prudential norms. ( $(14)$

\section{September 30, 2007}

Government adoption of a medium-term concept paper on rationalization of public employment consistent with the aim of limiting the general government wage bill to no more than 10 percent of GDP. ( 8 )

Adoption of an action plan to improve tax arrears management which we expect to implement by March 31, 2008, and which would involve enhancing a tax arrears accounting system to permit distinction of arrears on the basis of vintage, introducing prompt and unconditional measures to ensure forced collection of arrears, passing legislation for

\footnotetext{
${ }^{3}$ All conditions agreed in the MEFPs of April 14 and November 29, 2006, retain force, except as modified here.
} 
shortening and streamlining the procedure for writing off uncollectible tax arrears, and consolidating all domestic tax assessing and collecting activities in one agency. ( $(9)$

\section{Structural Benchmarks}

\section{Continuous}

Tariffs for consumption of natural gas and electricity to remain at cost-recovery levels. ( $₫ 10$ )

\section{September 30, 2007}

Draft 2008 budgetary documentation to include information on the financial performances for 2006 of state-owned enterprises and joint stock companies in which the state is holding a majority stake. ( $₫ 25)$

The ministry of finance and the NBM to adopt a plan to securitize the remaining outstanding stock of NBM claims on government. (\$13)

Government will adopt the methodology for a targeted social assistance system. ( $(10)$

Parliamentary passage of legislation ensuring that ANRE establishes tariffs for heat and water directly, rather than indirectly through the municipality as at present. ( $₫ 10)$

Increase household tariffs for heat and water sufficiently to reach a weighted average tariff paid by all categories of consumer of heat in Chişinău equal to 70 percent of cost recovery. (Ф10)

In accordance with the decision of the municipal council Nr.64/2 from January 25, 2007, the difference between the amount paid by households and the cost recovery tariff to be covered from the municipal budget. ( $₫ 10)$

\section{December 31, 2007}

Transfer of balances on accounts of Social Fund and Health Fund to Single Treasury Account in NBM. ( $(11)$

\section{March 31, 2008}

Outstanding stock of NBM claims on government fully securitized. (ף 13) 
Implementation of the system to modernize tax arrears management, including an enhanced tax arrears accounting system to permit distinction of arrears on the basis of vintage, introducing prompt and unconditional measures to ensure forced collection of arrears, passing legislation for shortening and streamlining the procedure for writing off uncollectible tax arrears, and consolidation of all tax assessing and collecting activities in one agency. ( $(9)$

\section{June 30, 2008}

Introduction of a system whereby the State Treasury ensures that the balances in commercial banks of the social insurance budget (BASS) and the mandatory health insurance fund are zero-ed out on a daily basis. ( $(24)$

\section{September 30, 2008}

Introduce the targeted social assistance system throughout Moldova. (ब 10) 


\section{AtTaChment III: TeChnical Memorandum OF Understanding}

1. This Technical Memorandum of understanding (TMU) defines the variables subject to quantitative targets (performance criteria and indicative benchmarks as shown in Table 1), established in the Memorandum of Economic and Financial Policies (MEFP) and describes the methods to be used in assessing the program performance with respect to these targets.

\section{Program Assumptions}

$$
2007
$$

2. Loan disbursements of $\$ 59.1$ million.

3. Receipts to the general government budget of privatization proceeds in the amount of MDL 235 million in 2007.

4. For program monitoring purposes, U.S. dollar denominated components of the NBM balance sheet will be valued at the program exchange rate. The program exchange rate of the Moldovan leu (MDL) to the U.S. dollar has been set at MDL 13.2911/\$. Amounts denominated in other currencies will be converted for program purposes into U.S. dollar amounts using the cross rates $\mathrm{USD} / €=1.2660, \mathrm{USD} / £=1.8702, \mathrm{SDR} / \mathrm{USD}=0.6773$.

5. To calculate the adjustments for disbursements from external sources exceeding the programmed amounts, the actual exchange rate at the time of the disbursement will be used. To calculate the adjustments for shortfalls of disbursement, the assumed exchange rate in the program for that disbursement will be used.

\section{REPORTING REQUIREMENTS}

6. Macroeconomic data necessary to assess performance criteria and indicative benchmarks to measure performance will be provided to Fund staff with including, but not limited to data as specified in Table 2 . The authorities will transmit promptly to Fund staff any data revisions. 


\section{Program Targets AND Definitions}

\section{Floor on the Stock of Net International Reserves (NIR)}

(In millions of lei)

\begin{tabular}{ll}
\hline Position on & \multicolumn{1}{c}{ Minimum Levels } \\
\hline March 31, 2007 & Net international reserves \\
June 30, 2007 & 8,500 (actual) \\
September 30, 2007 & 9,483 ( indicative target) \\
December 31, 2007 & 9,830 (performance criterion ) \\
\hline
\end{tabular}

7. Net international reserves of the NBM in convertible currencies are defined as gross reserves minus reserve liabilities in convertible currencies. For program monitoring purposes, gross reserves of the NBM are defined as monetary gold, holdings of SDRs, reserve position in the Fund, and holdings of foreign exchange in convertible currencies that are readily available and controlled by the NBM, including holdings of securities denominated in convertible currencies that are freely usable for settlement of international transactions, calculated using program assumptions on bilateral exchange rates. Excluded from reserve assets are capital subscriptions to foreign financial institutions, long-term nonfinancial assets, funds disbursed by the World Bank or other international institutions assigned for on-lending and project implementation, assets in nonconvertible currencies, and foreign assets pledged as collateral or otherwise encumbered, including claims in foreign exchange arising from transactions in derivative assets (futures, forwards, swaps, and options). Reserve liabilities in convertible currencies are defined as use of Fund credit, and convertible currency liabilities of the NBM to nonresidents with an original maturity of up to and including one year. Excluded from reserve liabilities are liabilities with original maturities longer than one year.

Ceilings on Reserve Money and the Net Domestic Assets (NDA) of the NBM

(In millions of lei)

\begin{tabular}{lll}
\hline Position on & \multicolumn{1}{c}{$\begin{array}{c}\text { Maximum level } \\
\text { NDA }\end{array}$} & $\begin{array}{c}\text { Maximum level } \\
\text { Reserve Money } \\
\text { (Indicative target) }\end{array}$ \\
\hline March 31, 2007 & $-2,003$ (actual) & 6,497 (actual) \\
June 30, 2007 & $-2,468$ (indicative target) & 7,014 \\
September 30, 2007 & $-2,606$ (performance criterion) & 7,223 \\
December 31, 2007 & $-3,261$ (indicative target) & 7,443 \\
\hline
\end{tabular}


8. Reserve money is defined as currency in circulation (outside banks), vault cash of banks, total required reserves, and balances on correspondent accounts of banks in the NBM in lei.

9. Net domestic assets of the NBM are defined as the difference between reserve money (defined in paragraph 8) and net foreign assets of the NBM.

10. Net foreign assets of the NBM are defined as gross reserves in convertible currencies (defined in paragraph 7) plus foreign assets in nonconvertible currencies, funds disbursed by the World Bank or other international institutions assigned for on-lending and project implementation, and foreign assets pledged as collateral or otherwise encumbered, including claims in foreign exchange arising from transactions in derivative assets, and net other foreign assets, minus foreign exchange liabilities of the NBM to nonresidents.

Floor on the Overall Cash Balance of the General Government

(In millions of lei)

\begin{tabular}{ll}
\hline & Cash balance \\
\hline Cumulative change from December 31, 2006 & \\
March 31, 2007 (performance criterion) & 386 (actual) \\
June 30, 2007 (indicative target) & -248 \\
September 30, 2007 (performance criterion) & -238 \\
December 31, 2007 (indicative target) & -233 \\
\hline
\end{tabular}

11. The general government is defined as comprising the central and local government budgets. The central government includes also the Social Insurance Fund, the Health Insurance Fund, special and extrabudgetary funds, and foreign-financed investment projects. The local government includes also special and extrabudgetary funds. The authorities will inform the Fund staff of any new special or extrabudgetary funds that may be created during the program period to carry out operations of a fiscal nature and will ensure that these will be included in the general government. Excluded are any government-owned entities with a separate legal status. Net credit of the banking system to general government is defined as outstanding claims of the banking system on the general government (exclusive of the claims associated with accrued interest, tax and social contribution payments by commercial banks, and foreign financed on-lending by banks), including overdrafts, direct credit and holdings of government securities, less deposits of the general government (excluding accrued interest on government deposits, and including the accounts for foreign-financed investment 
projects). ${ }^{4}$ The Ministry of Finance will provide data on the holdings of government securities and foreign-financed investment projects.

12. The quarterly limits on the overall cash deficit of the general government are cumulative and will be monitored from the financing side as the sum of net credit of the banking system to the general government (excluding the change in the stock of government securities issued to recapitalize the central bank), the general government's net placement of securities outside the domestic banking system, other net credit from the domestic nonbanking sector to the general government, the general government's receipt of disbursements from external $\mathrm{debt}^{5}$ for direct budgetary support and for specific projects minus amortization paid, and privatization proceeds stemming from the sale of the general government's assets, after deduction of the costs directly associated with the sale of these assets.

13. The quarterly limits on the general government wage bill are cumulative and measured as the sum of total salaries, bonus payments and other types of remuneration, social security contributions to the National Social Insurance House, and contributions to the National Health Insurance Company paid to all employees in the general government sector as defined in paragraph 11, excluding wages paid to employees of the National Social Insurance House and the National Health Insurance Company. For 2006, the general government wage bill based on such a definition amounted to 4,183 million lei. ${ }^{6}$

Ceiling on the General Government Wage Bill

(In millions of lei)

Indicative Target

Cumulative change from December 31, 2006

$\begin{array}{lr}\text { March 31, } 2007 \text { (actual) } & 1,093\end{array}$

June 30, 2007

2,430

September 30, 2007

3,635

December 31, 2007

4,966

\footnotetext{
${ }^{4}$ For the calculation of the net credit of the banking system to general government the following accounts will be excluded: 1731, 1732, 1733, 1735, 1761, 1762, 1763, 1801, 1802, 1805, 1807, 2711, 2717, 2721, 2727 , 2732, 2733, 2796, 2801 and 2802.

${ }^{5}$ Debt is defined as in footnote 3 in the section on limits on external debt.

${ }^{6}$ For the calculation of the total general government wage bill the following accounts for central government, local government, and special funds from the Treasury system in the Ministry of Finance will be used: 111, 112, 1161.
} 
14. Government securities in the form of zero-coupon obligations sold at a discount to face value will be treated as financing items in the fiscal accounts, in the amount actually received from buyers. At the time of redemption, the sales value will be recorded as amortization, and the difference between amortization so defined and the face value will be recorded as domestic interest payments.

15. External-debt limits apply to the contracting or guaranteeing of (i) short-term nonconcessional external debt (with an original maturity of up to and including one year) and (ii) non-concessional medium- and long-term debt with original maturities of more than one year. The limit is zero with the exception of the road project to be partly financed by $€ 30$ million from the European Investment Bank (EIB) and by $€ 30$ million from the European Bank for Reconstruction and Development (EBRD), as well as for the health project financed by $€ 9$ million from the Council of Europe Development Bank (CEB). The first phases of the EBRD and EIB road project (in the amount of $€ 12.5$ million each) will not have grant co-financing, but the second phases (in the amount of $€ 17.5$ million each) are expected to include grant co-financing sufficient to bring the overall level of concessionality on the second phases of the project to at least the target level of 35 percent. Short-term debt includes all short term obligations, excluding import trade credits. Shortterm debt denominated in currencies other than the U.S. dollar shall be valued in U.S. dollars at the exchange rate prevailing at the time of disbursement. Medium- and long-term debt denominated in currencies other than the U.S. dollar shall be valued in U.S. dollars at actual cross-exchange rates.

Ceilings on Contracting or Guaranteeing of Non-concessional External Debt of the General Government (In millions of Euro)

\begin{tabular}{lccc}
\hline & $\begin{array}{c}\text { EBRD/EIB Road } \\
\text { Rehabilitation project }\end{array}$ & $\begin{array}{c}\text { CEB Health } \\
\text { Project }\end{array}$ & Other \\
\hline Cumulative change from December 31, 2006 & 0 & 0 & 0 \\
March 31, 2007 (performance criterion) & 60 & 9 & 0 \\
June 30, 2007 (indicative target) & 60 & 9 & 0 \\
September 30, 2007 (performance criterion) & 60 & 9 & 0 \\
December 31, 2007 (indicative target) & & 9 & \\
\hline
\end{tabular}

16. The term debt has the meaning set forth in point No. 9 of the Guidelines on Performance Criteria with Respect to Foreign Debt (Decision No. 12274-(00/85), adopted August 24, 2000). ${ }^{7}$ This performance criterion applies not only to debt as defined above, but also to commitments contracted or guaranteed for which value has not been received.

\footnotetext{
${ }^{7}$ Debt is defined as a current, i.e., not contingent, liability, created under a contractual arrangement through the provision of value in the form of assets (including currency) or services, and which requires the obligor to make
} 
17. For purpose of the program, the guarantee of a debt arises from any explicit legal obligation of the government or the NBM or any other agency acting on behalf of the government to service such a debt in the event of nonpayment by the recipient.

18. Concessionality will be calculated using currency-specific discount rates based on the OECD commercial interest reference rates (CIRRs). The ten-year average of CIRRs will be used as the discount rate to assess the concessionality of loans of an original maturity of at least 15 years, and a six-month average of CIRRs will be used to assess the concessionality of loans with original maturities of less than 15 years. To both the ten-year and six-month averages, the following margins will be added: 0.75 percent for repayment periods of less than 15 years; 1 percent for 15-19 years; 1.15 percent for 20-30 years; and 1.25 percent for over 30 years. Under this definition, only loans with a grant element equivalent to 35 percent or more will be excluded from the borrowing limits. The debt limits will not apply to loans classified as international reserve liabilities of the NBM.

19. For the purposes of the program, external payments arrears will consist of all overdue debt-service obligations (i.e. payments of principal or interest) arising in respect of any debt contracted or guaranteed or assumed by the government of the Republic of Moldova, or the NBM, or any agency acting on behalf of the government of the Republic of Moldova. The ceiling on new external payments arrears shall apply on a continuous basis throughout the period of the arrangement. It shall not apply to external payments arrears arising from external debt being renegotiated with external creditors, including Paris Club creditors; and more specifically, to external payments arrears in respect of which a creditor has agreed that no payment needs to be made pending negotiations.

20. Expenditure arrears are defined as the difference between payment obligations due, and actual payments made. They can arise on any expenditure item, including transfers, debt service, wages, pensions, energy payments and goods and services. Expenditure arrears for goods and services to suppliers are defined as obligations to suppliers, which are due but not paid for more than 30 days and are non-disputed. Arrears between the state budget, local government, social and health funds, and all extrabudgetary funds are not counted towards the expenditure arrears' ceiling on the general government.

one or more payments in the form of assets (including currency) or services, at some future point(s) in time; these payments will discharge the principal and/or interest liabilities incurred under the contract. Arrears, penalties, and judicially awarded damages arising from the failure to make payment under a contractual obligation that constitutes debt are debt. Failure to make payment on an obligation that is not considered debt under this definition (e.g., payment on delivery) will not give rise to debt. 


\section{AdJUSTERS}

21. In the event that privatization receipts exceed the program assumptions, the limits on the overall cash deficit of the general government will be increased by the corresponding magnitude up to a cumulative cap of lei 125 million.

22. In case disbursements of external loans exceed the program assumptions, the limits on the overall cash deficit of the general government will be increased by the corresponding magnitude up to a cumulative cap of lei 188 million. In case of shortfalls, the limits will be decreased by the full amount.

23. The limits on the overall cash deficit of the general government will be increased by the amount of paid in cash for recapitalization of the NBM or by the face value of government securities issued for the same purpose. 


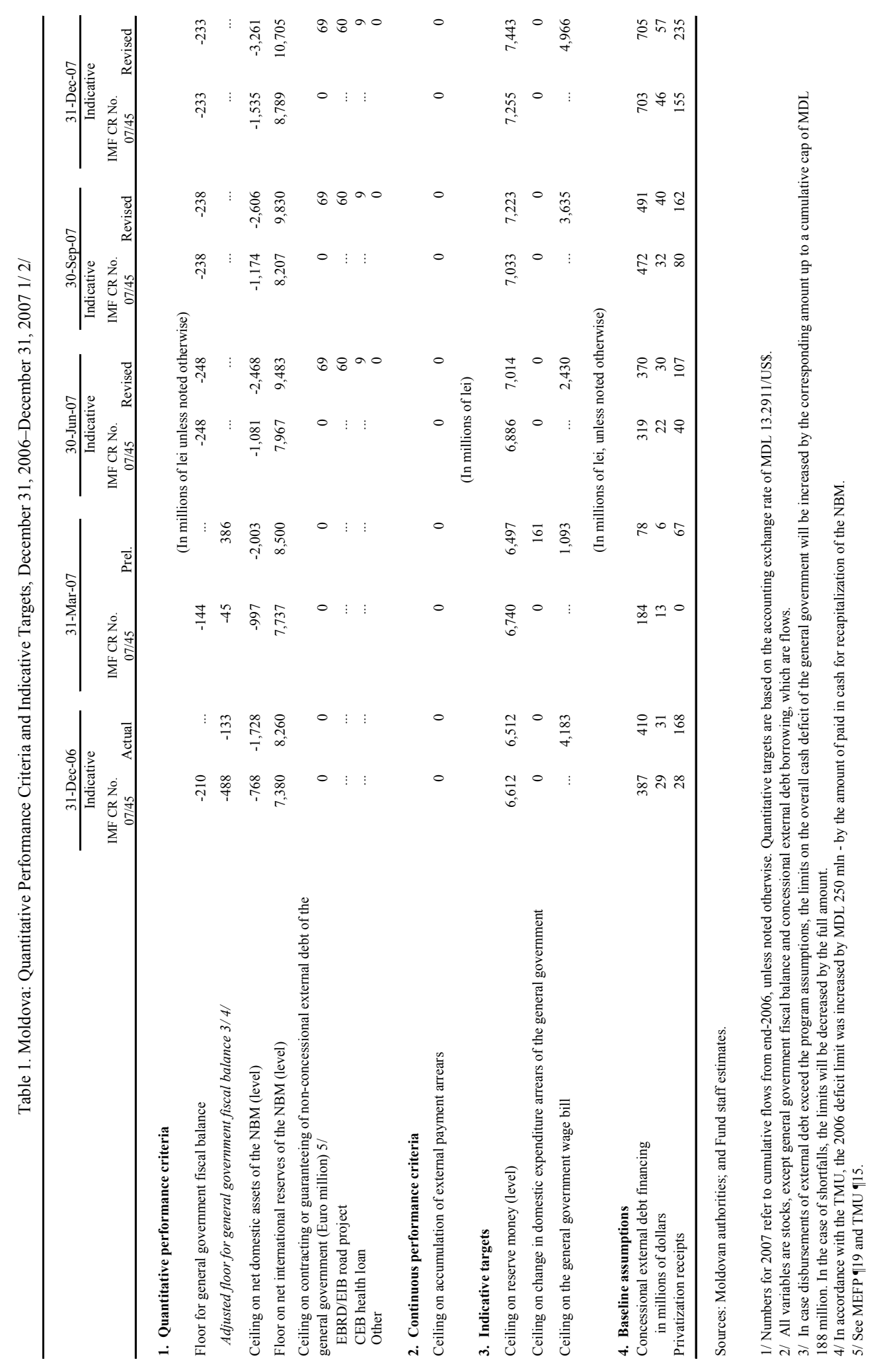


Table 2. Moldova: Data to be Reported to the IMF

\section{Item}

Fiscal data (to be provided by the MoF)

General budget operations for revenues, expenditure and financing (functional and economic).

General government wage bill

Domestic debt

Domestic arrears

Privatization receipts received by the budget (in lei and foreign exchange, net of divestiture transactions costs).

Monetary data (to be provided by the NBM)

Monetary survey of the NBM

Monetary survey for the whole banking system

Net claims on general government (NBM and commercial banks)

Financial indicators of commercial banks (from NBM's

Banking Supervision)

Foreign exchange cash flows

Foreign exchange operations (NBM data)

Foreign exchange market data (volume of trades, interventions, exchange rates)

NBM's sterilization operations

Interbank transactions (volumes, average rates)

Balance of Payments (to be provided by the NBM)

Current and capital account data.

Transfers/remittances through the banking system

External debt data (to be provided by MoF)

Information on all new external loans contracted by the government or government guarantee.

\section{Periodicity}

Monthly, within three weeks of the end of each month

Monthly, within three weeks of the end of each month

Monthly, within two weeks of the end of each month

Monthly, within three weeks of the end of each month

Monthly within three weeks of the end of each month

Weekly within one week of the end of each week Weekly within two weeks of the end of each week Weekly within two weeks of the end of each week

Monthly within four weeks of the end of each month

Monthly, within two weeks of the end of each month

Monthly, within two weeks of the end of each month

Daily within 12 hours of the end of each day

Weekly within one week of the end of each week

Weekly within one week of the end of each week

Quarterly within six weeks of the end of each quarter

Monthly within six weeks of the end of each month

Monthly within three weeks of the end of each month 
Item

Total debt service due by creditor, and debt service paid.

Disbursements of grants and loans by creditor

Other data (to be provided by NBS)

Overall consumer price index.

National accounts by sector of production, in nominal and real terms.

Export and import data on value, volume, and unit values, by major categories and countries.

\section{Periodicity}

Monthly within three weeks of the end of each month

Monthly, within three weeks of the end of each month

Monthly within two weeks of the end of each month.

Quarterly within three months of the end of each quarter.

Monthly within two months of the end of each month. 


\section{Moldova - Joint BANK-Fund DeBT SustainabiLity ANALYSIS ${ }^{1}$}

Based on the external LIC DSA, Moldova's risk of debt distress is low. The public DSA suggests that Moldova's overall public sector debt dynamics are sustainable in light of the current size and the evolution of the domestic debt stock. ${ }^{2}$

\section{A. Background}

1. Moldova's debt situation has improved since the last DSA in early 2006. At that time, short-term financing needs stemmed from the projected financing gaps from external shocks and the stock of arrears owed to bilateral creditors (mainly Russia, the US and Japan). The latter concerns were subsequently resolved through Paris Club rescheduling in May 2006. Debts covered under the agreement were 33 percent of Moldova's total debt stock, based on end-April 2006 numbers. ${ }^{3}$ However, the reduction in external debt was not as dramatic as it had been from 2000-2005: external debt was 124 percent of GDP in 2000 (with public debts totaling 95 percent of GDP), falling to 56 percent of GDP in 2005 (27 percent of GDP for public debt). ${ }^{4}$

2. Prospects for official donor assistance have also improved. In response to the twin external shocks - doubling of energy prices and Russia's ban on imports of Moldovan wine-large commitments were made by donors at the December 2006 Consultative Group meeting (primarily grants and technical assistance) and are expected to materialize over the next four years. Moldova has also qualified for grants from the Millennium Challenge Corporation, reducing the pressure to borrow for growth-enhancing investments. Finally, the PRGF arrangement was augmented at the time of the first review in December, raising the total amount of access to Fund resources from SDR 80.08 million to SDR 110.88 million.

\section{A significant fraction of external debt is on concessional terms, and will remain}

so. Table 1 illustrates the preponderance of debt owed to multilateral lenders on concessional terms and the likely evolution from future disbursements. Most borrowing in the near term is forecast to be on concessional terms, either directly or through integrated loan arrangements that incorporate grants. Over the longer term as Moldova graduates from IDA-only eligibility, borrowing will continue to be primarily from multilateral lenders, but will become less concessional. Loans from commercial creditors make up a very small fraction of the total.

\footnotetext{
${ }^{1}$ Data and analysis exclude Transnistria.

${ }^{2}$ The DSA has been produced jointly by the Bank and the Fund, in consultation with the EBRD.

${ }^{3}$ Moldova owed Germany, Italy, Japan, Russia, and the US a total of \$274 million, out of \$822 million.

${ }^{4}$ The rapid fall in debt to GDP was mainly due to strong GDP growth; the nominal amount of debt remained relatively constant.
} 


\begin{tabular}{|c|c|c|c|c|}
\hline \multicolumn{5}{|c|}{ Table 1: External Public Debt Profile } \\
\hline & \multicolumn{2}{|c|}{2007} & \multicolumn{2}{|c|}{$2008-272 /$} \\
\hline & Total & Concessional $1 /$ & Average & Concessional 1/ \\
\hline Debt stock (US\$): & 945.8 & 402.6 & 944.9 & 322.7 \\
\hline Multilateral & 618.8 & 402.6 & 673.5 & 273.4 \\
\hline World Bank & 408.4 & 272.0 & 510.2 & 243.1 \\
\hline IMF & 169.0 & 114.2 & 64.8 & 25.3 \\
\hline Others & 41.4 & 16.4 & 98.5 & 5.0 \\
\hline Bilateral & 304.2 & 0.0 & 263.2 & 49.3 \\
\hline Commercial & 22.8 & 0.0 & 8.2 & 0.0 \\
\hline New disbursements (US\$): & 96.7 & 88.1 & 53.7 & 19.2 \\
\hline Multilateral & 93.6 & 88.1 & 49 & 14.5 \\
\hline World Bank & 34.2 & 34.2 & 36.5 & 9.0 \\
\hline IMF & 49.8 & 49.8 & 2.5 & 2.5 \\
\hline Others & 9.6 & 4.1 & 10.0 & 3.0 \\
\hline Bilateral & 1.8 & 0.0 & 4.7 & 4.7 \\
\hline Commercial & 1.3 & 0.0 & 0.0 & 0.0 \\
\hline
\end{tabular}

\section{Private debt is growing, with loans associated with FDI becoming more}

important. Private external debt can be divided between regular loans (classified as liabilities under other capital in the capital account), loans associated with FDI transactions, and energy arrears. In 2001 new disbursements from FDI-linked loans comprised 35 percent of new borrowing, but by 2006 the proportion was 52 percent. Private debt is also increasingly denominated in euros - 20 percent in 2006 compared with only 6 percent in 2002. This is a welcome trend, given the natural hedge from remittances denominated in euros.

5. The increase in energy prices led to private external arrears. There is a large outstanding stock of historic debt from unpaid energy bills (totaling \$286 million at end2005), but in recent years current payments at least have been made. However, while electricity and natural gas tariffs are set at cost recovery, tariffs for heat are below that level causing Moldovagaz to run arrears of about \$34 million to Gazprom in 2006, and early indications are that arrears will continue in 2007 . Heating tariffs are now set by an independent regulator, and are scheduled to rise to full cost-recovery levels over the course of the program.

\section{B. Underlying Assumptions}

\section{Box 1 summarizes the medium-term macroeconomic framework underlying the}

DSA. Of particular importance is the assumption that remittances will continue to finance the balance of payments. Moldova has one of the highest proportions of remittances to GDP in the world, 33 percent in 2006. Nominal growth of remittances (in US\$) has averaged 
40 percent since 2001, reaching a peak of 54 percent in 2003 and subsiding to 29 percent in 2006. Strong growth in the euro area provides incentives to work abroad, but border crossings are now complicated by the accession of Romania to the EU at the beginning of the year (because of new visa requirements). Staff project remittance growth of 20 percent in 2007, and growth sufficient to keep remittances around 30 percent of GDP for the foreseeable future.

\section{The overall fiscal deficit is expected to remain at about 0.5 percent of GDP over} the medium term, permitting modest primary surpluses to accumulate. High levels of remittances should continue to feed import growth, VAT and excises on which will bolster revenue. Recent policies, such as an increase in civil service wages are expected to be offset by a medium-term rationalization of public sector employment. Despite the increase in donor assistance for capital expenditures over the next few years, there is not a large growth dividend built into the baseline scenario. Indeed, growth of 5 percent over the medium term assumes a mild deceleration from the pace of the past few years.

\section{Box 1. Moldova - Macroeconomic Assumptions}

The macroeconomic assumptions have not changed significantly since the last DSA: growth will remain strong, inflation to subside, with continuing current account deficits and primary government surpluses.

Real GDP growth is projected to average 5 percent through 2012 and falling to 4 percent thereafter. While this is higher than the historical average, the past decade included two years of severe recession in the aftermath of the Russian crisis of 1998 causing average growth rates to fall and volatility to rise. In the post-crisis period (2001-2006) real growth averaged 6.6 percent.

Inflation is projected to subside to the single-digit range by end-2007, falling to three percent by 2027 (as measured by the GDP deflator). This is considerably lower than the historical average of 16 percent, but measures taken under the program should ensure the National Bank of Moldova keeps a tight rein on monetary policy.

The current account is projected to continue to be supported by strong remittances, which in 2006 reached 33 percent of GDP. As remittances largely feed into consumption and imports, changes in flows largely net out with respect to the current account deficit. The improved prospects for the business environment have also raised capital inflows, in particular direct investment. Exports, which were down sharply after the Russian ban on wine, are projected to recover only gradually, as a mechanism for resuming exports has not yet been arranged, despite the formal lifting of the ban.

The overall fiscal balance is expected to remain negative, but with a small deficit. Grant assistance from donors, especially the European Union, is projected to increase, as are project loans that flow through the budget. This fiscal stance reflects the need to increase capital investment and social spending over the medium-term.

8. External public debt and debt-service ratios remain well below the thresholds in the baseline and historical scenarios. External debt and debt-service to exports ratios are 
healthy, despite the continuing effects of the Russian ban on wine (Figure 1). Included in the baseline are higher disbursements of concessional loans over the next four years. An alternative scenario (A2 in Table 1b) that examines the effects of less concessional terms on loans indicates no breach of the thresholds either.

9. A more worrisome possibility is a fall in transfers. An alternative scenario (B4 in Table 1b) shows the effects of a shock of one standard deviation below the historical average of net non-debt generating inflows in 2008-09 (which are primarily remittances in Moldova). The thresholds for debt to GDP and debt to exports are breached and do not fall back to more sustainable levels until 2012 and 2014, respectively. This illustrates Moldova's dependence on remittances. The baseline scenario assumes that remittances plateau at the current percent of GDP. However, should the labor environment abroad become less welcoming, or a slowdown in Russia or the EU (the two main destinations) occur, migrants may find it more difficult to maintain their level of support to dependants in Moldova. On the other hand, as remittance flows tend to be much less volatile than other types of inflows, such as portfolio investment, the chances for them to dry up appear slim. ${ }^{5}$

Table 2: Policy-Based External Public Debt Burden Indicators

\begin{tabular}{lrrr}
\hline & Threshold 1/ & 2007 & 2008-27 2/ \\
\hline NPV of external debt in percent of: & & & \\
Exports & 100 & 51.7 & 23.6 \\
GDP & 30 & 22.7 & 9.5 \\
Revenues & 200 & 68.9 & 26.2
\end{tabular}

External Debt Service in percent of:

$\begin{array}{llll}\text { Exports } & 15 & 3.7 & 1.5 \\ \text { Revenues } & 25 & 7.5 & 2.7\end{array}$

1/ Policy-dependent thresholds as used in the joint IMF-World Bank LIC DSA framework for a poor performer. Moldova's ratings have been improving, but classifications are based on three-year moving averages.

2/ Simple average.

\section{Public Debt Sustainability}

\section{Public debt and debt-service to revenue ratios remain well below the} appropriate thresholds in both the baseline and historical scenarios. Public domestic debt has remained steady at about 25 percent of total public debt, and is held primarily by the National Bank of Moldova. As there were primary surpluses in recent years, the historical scenario shows how quickly all public debt could be paid off: the debt and debt-service ratios

\footnotetext{
${ }^{5}$ While the annual growth rate of remittances has been over 30 percent since 2001, experiences from other countries do not imply equal risks of large negative growth. In addition, remittance growth in the first quarter of 2007 is estimated at 29 percent, and the robust growth in the euro area is an upside risk.
} 
all become negative before 2020. In practice, this is unlikely to occur, especially considering Moldova's significant and widely-recognized needs to upgrade infrastructure and make other capital investments to lay the foundation for future growth.

11. A recession in 2008-09 would derail the baseline scenario. As shown in Figure 2 (and B1 in Table 2b), a contraction of 1.6 percent in 2008 and 2009 (stemming from a growth shock of one standard deviation below the historical average) would imply debt to GDP above 30 percent by 2011, and continuing to rise afterwards. This scenario assumes that government expenditures continue on their previous trajectory despite the recession. The recession implies that expenditure as a percent of GDP would rise, bringing the deficit to a permanently higher level. Moldova has a relatively high level of government expenditure as a proportion of GDP to begin with, and it has been increasing rapidly in recent years. Arresting this expansion is crucial to maintaining resilience to potential shocks. The growth forecast is good, and Moldova was fortunate that the twin shocks from the wine ban and higher energy prices did not adversely affect growth more. While somewhat extreme, the alternative growth scenario shows how a temporary shock would affect debt sustainability.

\section{E. Conclusions}

12. Moldova's debt outlook is favorable, with a low risk of debt distress. In the face of some severe shocks in 2006, the Moldovan authorities maintained policies that did not allow the debt situation to deteriorate. This led to an agreement with the Paris Club and commitments by the Consultative Group that have bolstered debt sustainability. Given Moldova's development needs, there appears to be room for modest additional borrowing for infrastructure and other high priority projects, as long as such borrowing remains prudent. Absorptive capacity will likely be a more binding constraint than debt sustainability, and since Moldova is forecast to graduate from IDA-only eligibility in a few years, opportunities for concessional borrowing should be seized in the near term.

13. Although the risk is low, there are some fragilities. The external outlook remains highly dependent on remittances, which have expanded rapidly in recent years. An interruption in the ability of migrants to support dependents in Moldova would significantly affect the sustainability of debt. Improving the business climate so that internal growth can attract migrants back into the country would improve resilience. In addition, a temporary recession would cause the public fiscal situation to deteriorate rapidly. A smaller government as a proportion of GDP would be less likely to endanger debt sustainability. 


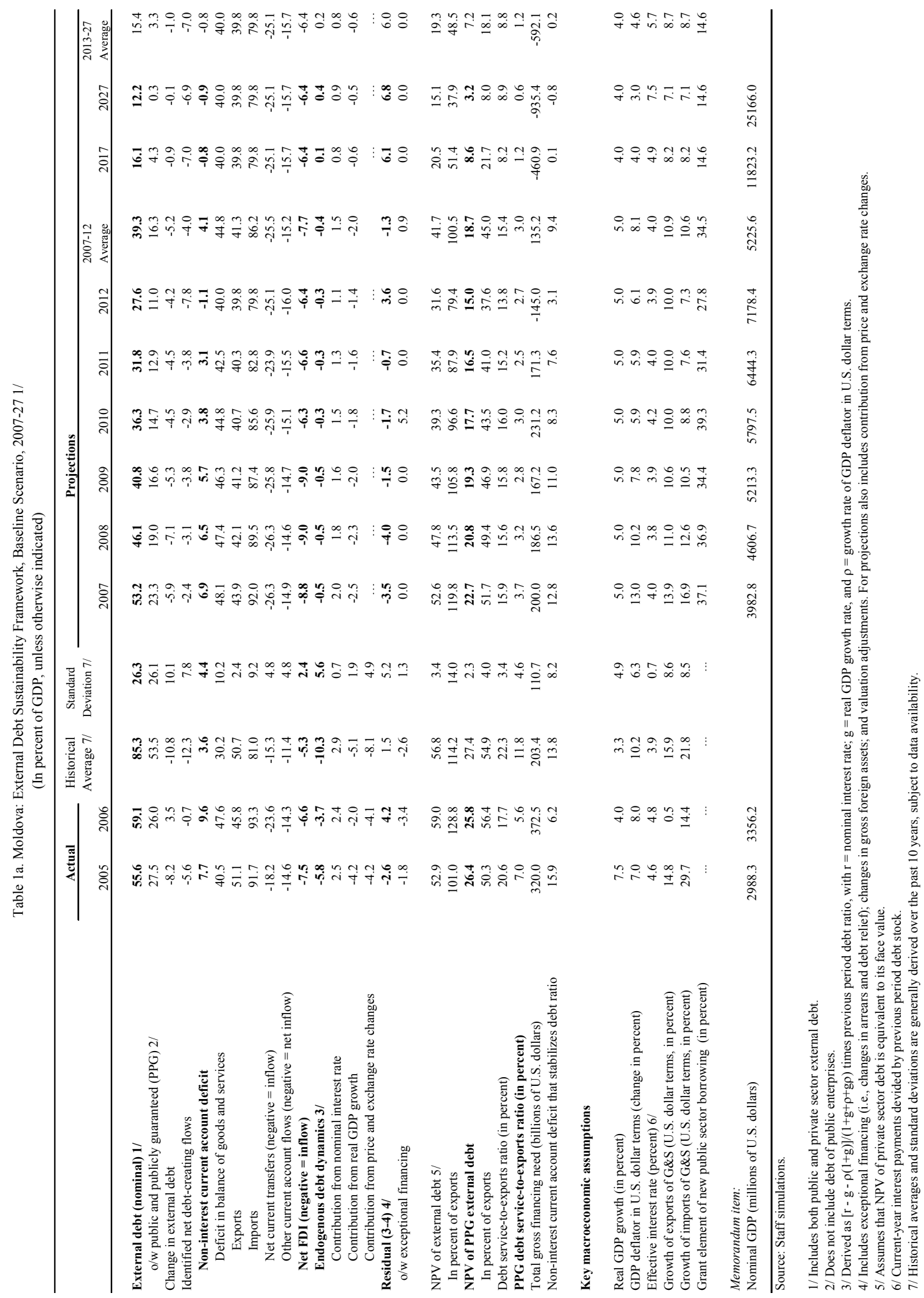


Table 1b. Moldova: Sensitivity Analyses for Key Indicators of Public and Publicly Guaranteed External Debt, 2007-27 1/ (In percent)

\begin{tabular}{|c|c|c|c|c|c|c|c|c|}
\hline & \multicolumn{8}{|c|}{ Projections } \\
\hline & 2007 & 2008 & 2009 & 2010 & 2011 & 2012 & 2017 & 2027 \\
\hline \multicolumn{9}{|c|}{ NPV of debt-to-GDP ratio } \\
\hline Baseline & 23 & 21 & 19 & 18 & 17 & 15 & 9 & 3 \\
\hline \multicolumn{9}{|l|}{ A. Alternative Scenarios } \\
\hline A1. Key variables at their historical averages in 2007-27 2/ & 23 & 21 & 20 & 18 & 17 & 19 & 26 & 28 \\
\hline A2. New public sector loans on less favorable terms in $2007-273$ / & 23 & 21 & 20 & 19 & 18 & 16 & 10 & 4 \\
\hline \multicolumn{9}{|l|}{ B. Bound Tests } \\
\hline B1. Real GDP growth at historical average minus one standard deviation in 2007-08 & 23 & 22 & 22 & 20 & 19 & 17 & 10 & 4 \\
\hline B2. Export value growth at historical average minus one standard deviation in 2007-08 4/ & 23 & 22 & 22 & 21 & 19 & 18 & 10 & 3 \\
\hline B3. U.S. dollar GDP deflator at historical average minus one standard deviation in $2007-08$ & 23 & 22 & 21 & 19 & 18 & 16 & 9 & 4 \\
\hline B4. Net non-debt creating flows at historical average minus one standard deviation in 2007-08 5/ & 23 & 36 & 49 & 45 & 42 & 39 & 20 & 4 \\
\hline B5. Combination of B1-B4 using one-half standard deviation shocks & 23 & 33 & 43 & 40 & 37 & 34 & 18 & 4 \\
\hline B6. One-time 30 percent nominal depreciation relative to the baseline in 2007 6/ & 23 & 29 & 27 & 24 & 23 & 21 & 12 & 4 \\
\hline \multicolumn{9}{|c|}{ NPV of debt-to-exports ratio } \\
\hline Baseline & 52 & 49 & 47 & 43 & 41 & 38 & 22 & 8 \\
\hline \multicolumn{9}{|l|}{ A. Alternative Scenarios } \\
\hline A1. Key variables at their historical averages in 2007-27 2/ & 52 & 50 & 49 & 44 & 43 & 48 & 65 & 71 \\
\hline A2. New public sector loans on less favorable terms in 2007-27 3/ & 52 & 50 & 49 & 46 & 44 & 41 & 25 & 11 \\
\hline \multicolumn{9}{|l|}{ B. Bound Tests } \\
\hline B1. Real GDP growth at historical average minus one standard deviation in 2007-08 & 52 & 49 & 47 & 43 & 41 & 38 & 22 & 8 \\
\hline B2. Export value growth at historical average minus one standard deviation in 2007-08 4/ & 52 & 54 & 58 & 54 & 51 & 47 & 26 & 9 \\
\hline B3. U.S. dollar GDP deflator at historical average minus one standard deviation in 2007-08 & 52 & 49 & 47 & 43 & 41 & 38 & 22 & 8 \\
\hline B4. Net non-debt creating flows at historical average minus one standard deviation in 2007-08 5/ & 52 & 86 & 118 & 111 & 105 & 98 & 51 & 10 \\
\hline B5. Combination of B1-B4 using one-half standard deviation shocks & 52 & 72 & 91 & 86 & 81 & 76 & 40 & 9 \\
\hline B6. One-time 30 percent nominal depreciation relative to the baseline in 2007 6/ & 52 & 49 & 47 & 43 & 41 & 38 & 22 & 8 \\
\hline
\end{tabular}

\section{Debt service ratio}

Baseline

A. Alternative Scenarios

A1. Key variables at their historical averages in 2007-27 2/

A2. New public sector loans on less favorable terms in $2007-273$

B. Bound Tests

B1. Real GDP growth at historical average minus one standard deviation in 2007-08

B2. Export value growth at historical average minus one standard deviation in 2007-08 4/

B3. U.S. dollar GDP deflator at historical average minus one standard deviation in 2007-08

B4. Net non-debt creating flows at historical average minus one standard deviation in 2007-08 5/

B5. Combination of B1-B4 using one-half standard deviation shocks

B6. One-time 30 percent nominal depreciation relative to the baseline in $20076 /$

Memorandum item:

Grant element assumed on residual financing (i.e., financing required above baseline) 7/

Source: Staff projections and simulations.

1/ Does not include debt of public enterprises.

2/ Variables include real GDP growth, growth of GDP deflator (in U.S. dollar terms), non-interest current account in percent of GDP, and non-debt creating flows.

3 / Assumes that the interest rate on new borrowing is by 2 percentage points higher than in the baseline, while grace and maturity periods are the same as in the baseline.

4/ Exports values are assumed to remain permanently at the lower level, but the current account as a share of GDP is assumed to return to its baseline level after the shock (implicitly assuming an offsetting adjustment in import levels).

5/ Includes official and private transfers and FDI.

6/ Depreciation is defined as percentage decline in dollar/local currency rate, such that it never exceeds 100 percent.

7/ Applies to all stress scenarios except for A2 (less favorable financing) in which the terms on all new financing are as specified in footnote 2. 


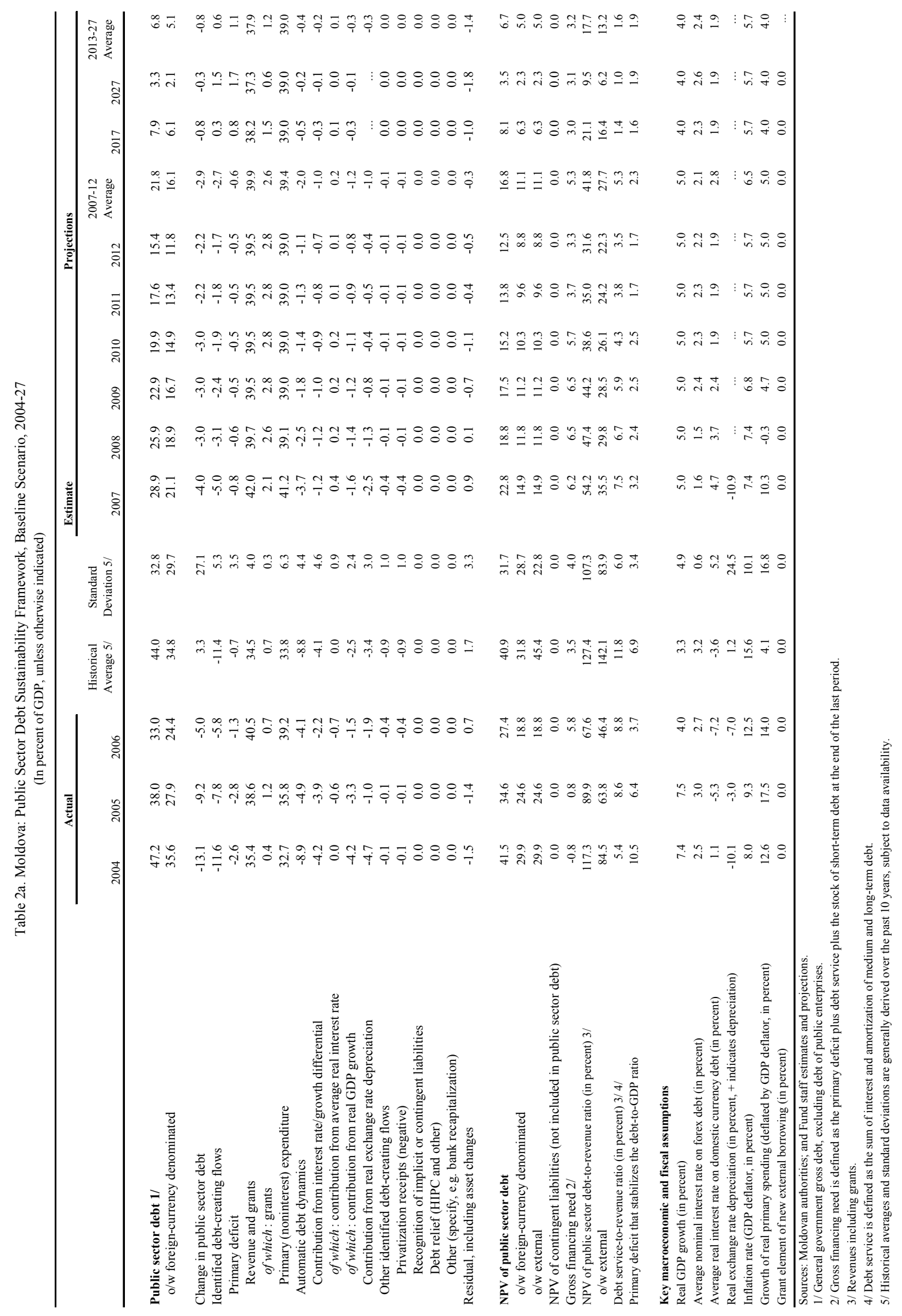


Table 2b. Moldova: Sensitivity Analysis for Key Indicators of Public Debt 2007-27

NPV of Debt-to-GDP Ratio
Baseline
A. Alternative scenarios
A1. Real GDP growth and primary balance are at historical averages
A2. Primary balance is unchanged from 2007
A3. Permanently lower GDP growth 1/
B. Bound tests
B1. Real GDP growth is at historical average minus one standard deviations in 2008-09
B2. Primary balance is at historical average minus one standard deviations in 2008-09
B3. Combination of B1-B2 using one half standard deviation shocks
B4. One-time 30 percent real depreciation in 2008
B5. 10 percent of GDP increase in other debt-creating flows in 2008
Baseline
A. Alternative scenarios
A1. Real GDP growth and primary balance are at historical averages
A2. Primary balance is unchanged from 2007
A3. Permanently lower GDP growth 1/
B. Bound tests
B1. Real GDP growth is at historical average minus one standard deviations in 2008-09
B2. Primary balance is at historical average minus one standard deviations in 2008-09
B3. Combination of B1-B2 using one half standard deviation shocks
B5. 10 percent of GDP increase in other debt-creating flows in 2008
Not-to-Revenue Ratio 2/

\begin{tabular}{llllllll}
\multicolumn{7}{c}{ Projections } \\
2007 & 2008 & 2009 & 2010 & 2011 & 2012 & 2017 & 2027 \\
\hline
\end{tabular}

$\begin{array}{rrrrrrrr}23 & 19 & 17 & 15 & 14 & 12 & 8 & 4 \\ & & & & & & & \\ 23 & 19 & 18 & 16 & 14 & 13 & 3 & -17 \\ 23 & 19 & 17 & 14 & 13 & 11 & 2 & -17 \\ 23 & 19 & 19 & 18 & 18 & 18 & 27 & 69\end{array}$

Debt Service-to-Revenue Ratio 2/

\section{Baseline}

\section{A. Alternative scenarios}

A1. Real GDP growth and primary balance are at historical averages

A2. Primary balance is unchanged from 2007

A3. Permanently lower GDP growth $1 /$

\section{B. Bound tests}

B1. Real GDP growth is at historical average minus one standard deviations in 2008-09 B2. Primary balance is at historical average minus one standard deviations in 2008-09

$\mathrm{B} 3$. Combination of B1-B2 using one half standard deviation shocks

B4. One-time 30 percent real depreciation in 2008

B5. 10 percent of GDP increase in other debt-creating flows in 2008

$\begin{array}{rrrrrrrr}23 & 22 & 27 & 29 & 31 & 34 & 46 & 64 \\ 23 & 22 & 23 & 21 & 19 & 17 & 12 & 6 \\ 23 & 21 & 22 & 19 & 17 & 15 & 9 & 3 \\ 23 & 24 & 22 & 20 & 18 & 17 & 12 & 6 \\ 23 & 28 & 26 & 23 & 22 & 20 & 14 & 7\end{array}$

$\begin{array}{llllllll}54 & 47 & 44 & 39 & 35 & 32 & 21 & 9\end{array}$

$\begin{array}{rrrrrrrr}54 & 48 & 45 & 40 & 36 & 33 & 9 & -45 \\ 54 & 47 & 43 & 37 & 32 & 28 & 4 & -45 \\ 54 & 49 & 48 & 45 & 45 & 46 & 71 & 186\end{array}$

$\begin{array}{rrrrrrrr}54 & 56 & 68 & 72 & 78 & 84 & 119 & 171 \\ 54 & 55 & 59 & 52 & 48 & 44 & 31 & 15 \\ 54 & 53 & 55 & 48 & 43 & 39 & 25 & 8 \\ 54 & 60 & 57 & 51 & 47 & 43 & 32 & 15 \\ 54 & 71 & 66 & 59 & 55 & 50 & 36 & 18\end{array}$

$\begin{array}{llllllll}8 & 7 & 6 & 4 & 4 & 3 & 1 & 1\end{array}$

Sources: Moldovan authorities; and Fund staff estimates and projections.

1/ Assumes that real GDP growth is at baseline minus one standard deviation divided by the square root of 20 (i.e., the length of the projection period).

2/ Revenues are defined inclusive of grants. 
Figure 1. Moldova: Indicators of Public and Publicly Guaranteed External Debt Under Alternative Scenarios, 2007-27

(In percent)

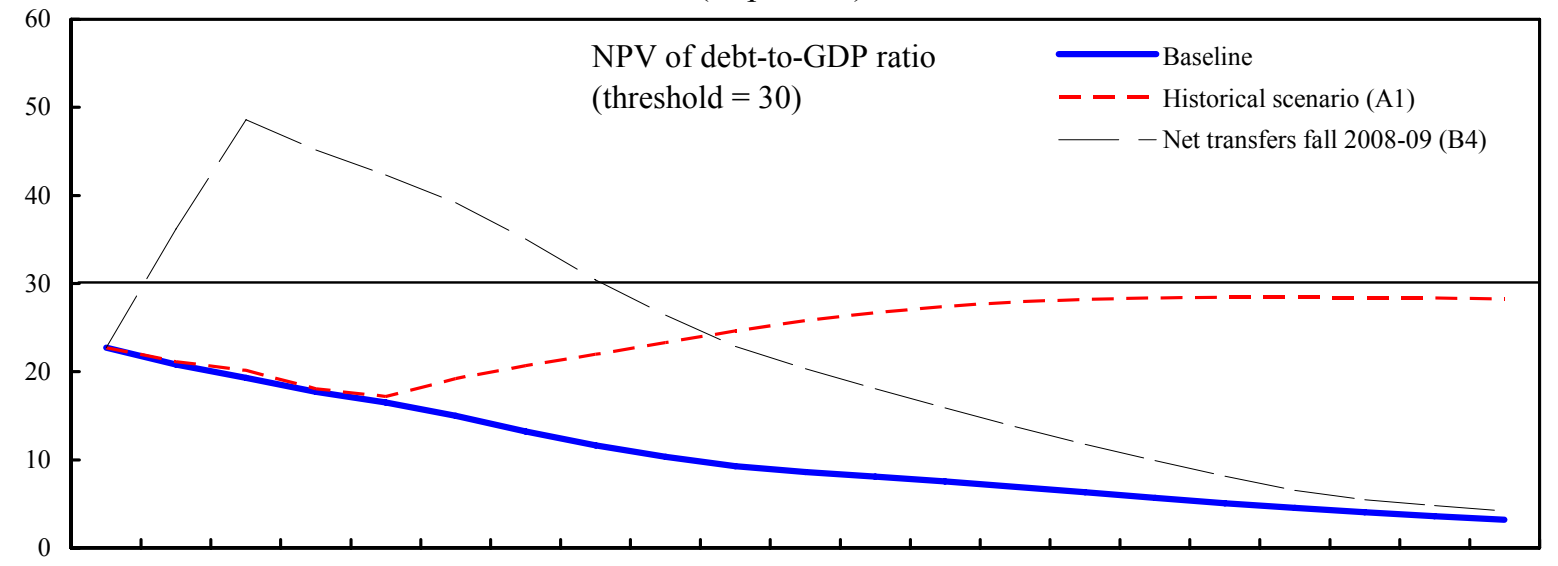

$2007200820092010201120122013201420152016201720182019202020212022 \quad 20232024202520262027$
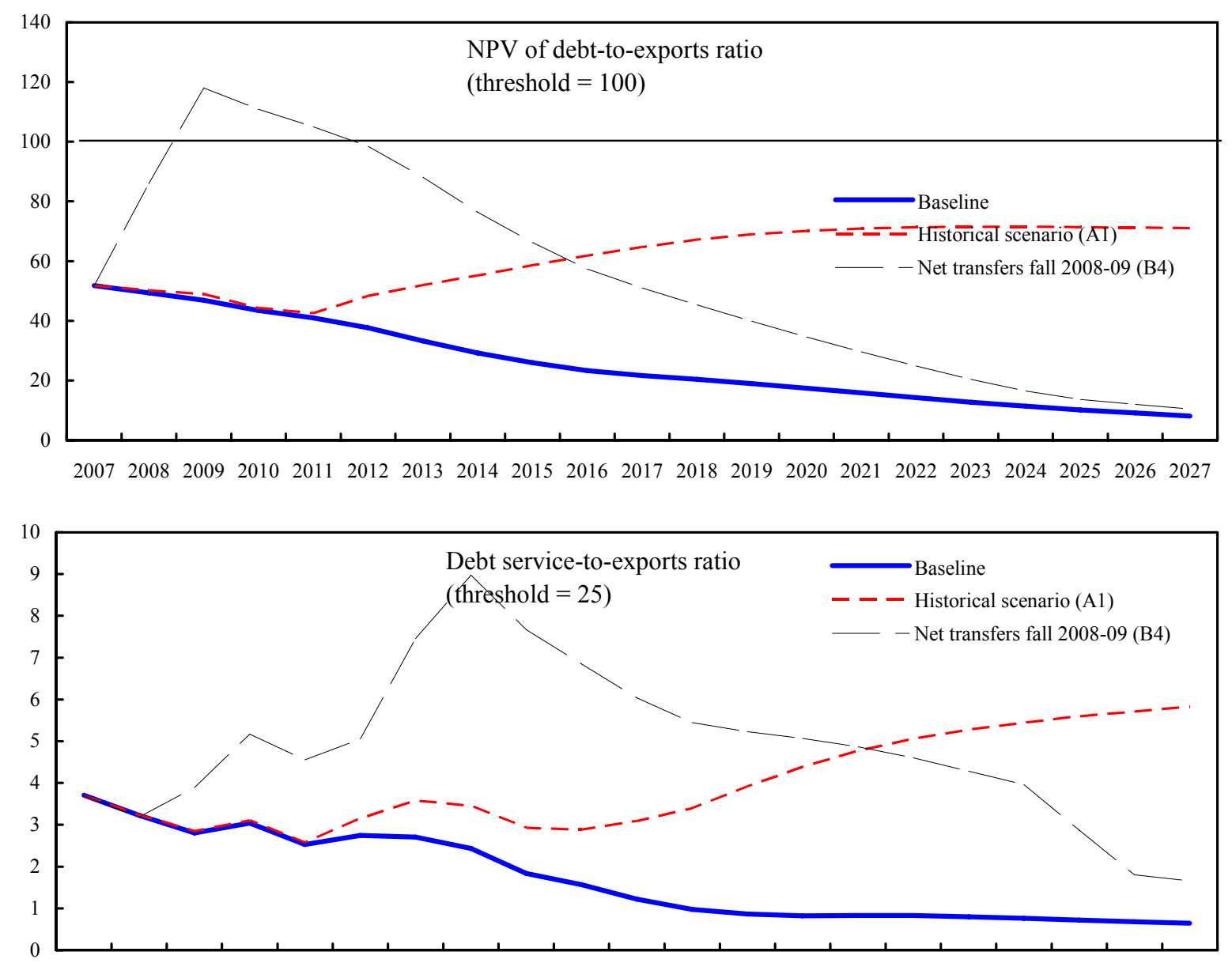

200720082009201020112012201320142015201620172018201920202021202220232024202520262027

Source: Staff projections and simulations. 
Figure 2. Moldova: Indicators of Public Debt Under Alternative Scenarios, 2007-27 1/

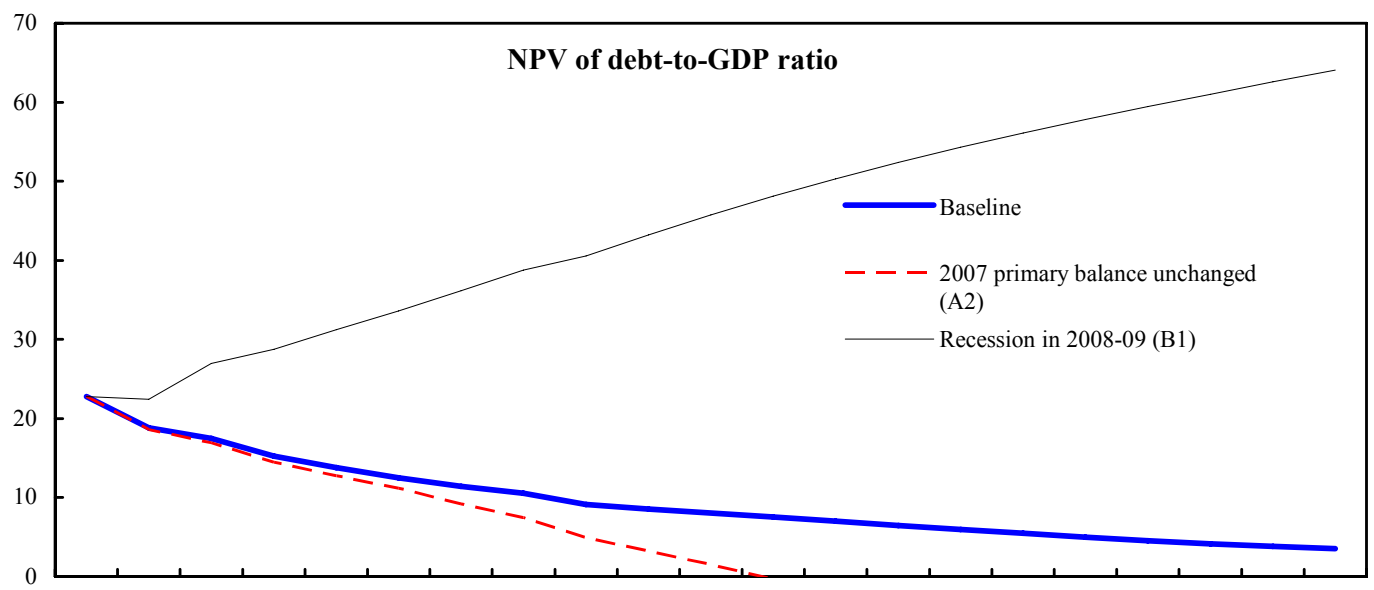

200720082009201020112012201320142015201620172018201920202021202220232024202520262027

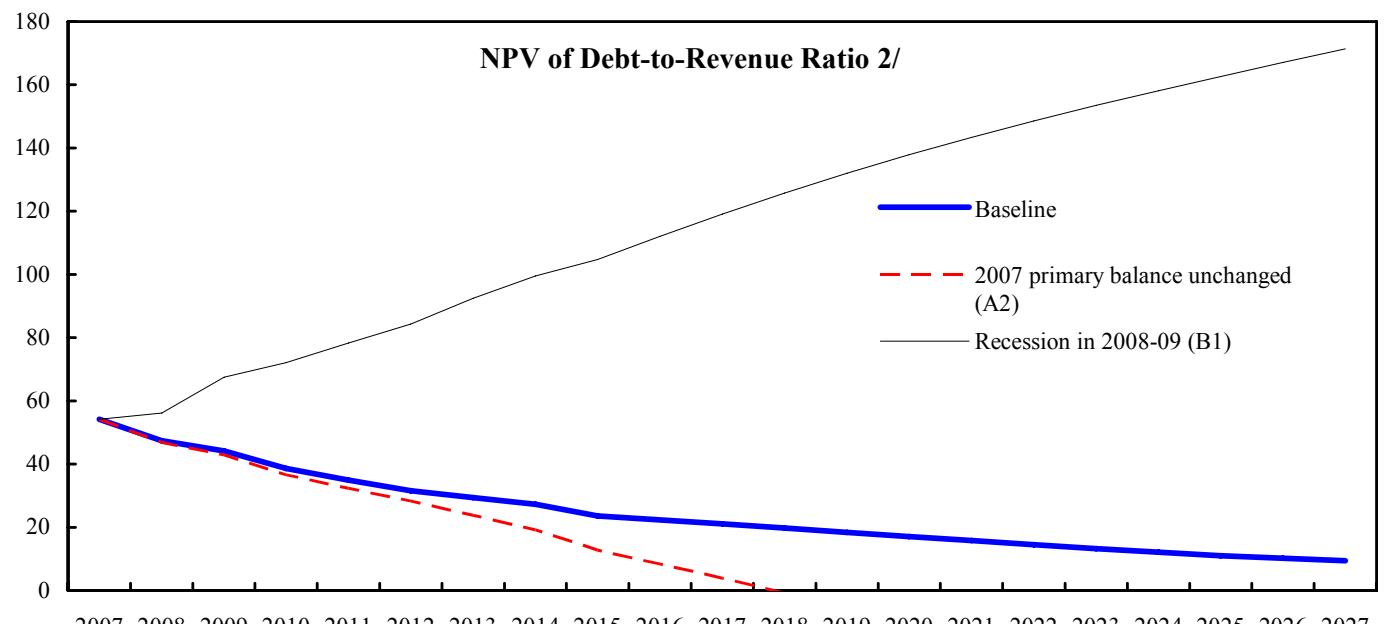

200720082009201020112012201320142015201620172018201920202021202220232024202520262027

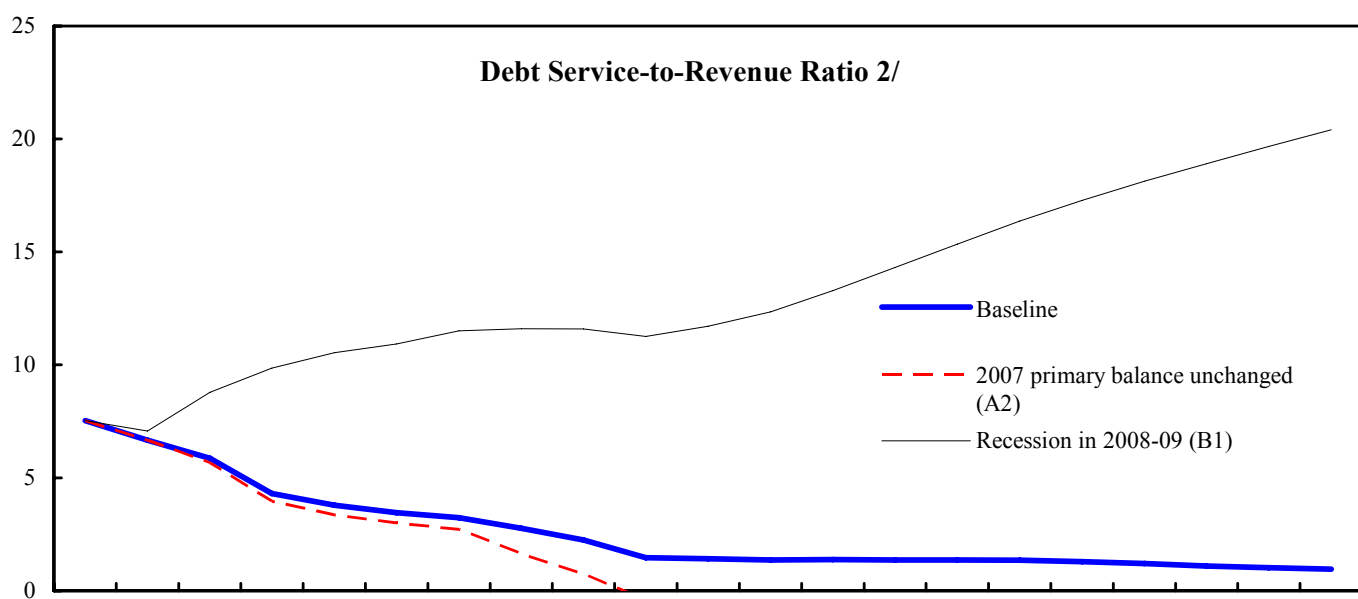

200720082009201020112012201320142015201620172018201920202021202220232024202520262027

Source: Staff projections and simulations.

1/ Most extreme stress test is test that yields highest ratio in 2017.

$2 /$ Revenue including grants. 



\title{
INTERNATIONAL MONETARY FUND
}

\section{REPUBLIC OF MOLDOVA}

\section{Second Review Under the Three-Year Arrangement Under the Poverty Reduction and Growth Facility and Requests for Waiver of Performance Criterion}

\author{
Informational Annexes \\ Prepared by the European Department \\ (In consultation with other departments and the World Bank)
}

June 25,2007

\begin{abstract}
- $\quad$ Discussions took place in Chişinău during April 25-May 8, 2007. The staff team included T. Richardson (head), N. Gigineishvili, S. Hida, M. Piatkowski (all EUR), I. Asmundson (PDR) and J. Mathisen (resident representative), and was supported by O. Scerbatchi, V. Buicli, and N. Culava of the Fund's Chişinău office. V. Munteanu, Advisor to the Executive Director, participated in the discussions. The mission met with the President, senior government and National Bank officials, members of parliament, representatives of the business and financial sectors, diplomats, donors, trade unions, and NGOs.

- $\quad$ The three-year PRGF was approved on May 5, 2006, with cumulative access of SDR 80.08 million (65 percent of quota), and SDR 11.44 million was disbursed upon program approval. Owing to external shocks faced by Moldova, and in light of the authorities' adjustment efforts, cumulative access was increased to SDR 110.88 million (90 percent of quota) at the first review, and SDR 31.97 million was disbursed following completion of the review on December 15, 2006 (IMF Country Report No. 07/ 45).

- $\quad$ The attached Letter of Intent and Memorandum of Economic and Financial Policies (Attachment) set out the authorities' economic program for the balance of 2007 and the medium term. The authorities have requested completion of the second review and a waiver for nonobservance of an end-December structural performance criterion.

- $\quad$ The authorities have agreed to publication of the program documents. The joint Bank-Fund DSA will be issued as a supplement, as will the appendices covering IMF relations, recent technical assistance and World Bank relations. These documents and the data therein do not cover the Moldovan region of Transnistria.

- $\quad$ In April 2006, the authorities' Economic Growth and Poverty Reduction Strategy Paper (EGPRSP) annual progress report for 2005 was issued to the IMF and World Bank Executive Boards, along with the Joint Staff Advisory Note (IMF Country Reports No. 06/185 and 186). A progress report for 2006 is expected to be finalized by autumn, along with the National Development Plan for 2008-10 that will replace the current EGPRSP.
\end{abstract}




\section{Contents}

\section{Page}

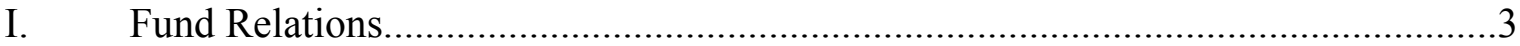

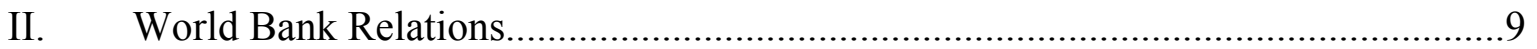

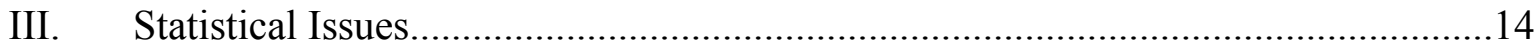


ANNEX I: MOLDOVA-FUND RELATIONS

(As of April 30, 2007)

I. Membership Status: Joined August 12, 1992; Article VIII

II. General Resources Account:

Quota

Fund holdings of currency

Reserve tranche position

III. SDR Department:

Holdings

IV. Outstanding Purchases and Loans:

Extended arrangements

PRGF arrangements
SDR million

123.20

143.20 .

0.01
Percent of Quota

100.00

116.23

0.00

SDR million Percent of Allocation

0.37

N/A

SDR million

20.00

Percent of Quota

16.23

67.43

\section{Latest Financial Arrangements:}

\begin{tabular}{lcccc}
\hline Type & $\begin{array}{c}\text { Approval } \\
\text { date }\end{array}$ & $\begin{array}{c}\text { Expiration } \\
\text { date }\end{array}$ & $\begin{array}{c}\text { Amount approved } \\
\text { (SDR million) }\end{array}$ & $\begin{array}{c}\text { Amount drawn } \\
\text { (SDR million) }\end{array}$ \\
\hline PRGF & $5 / 5 / 2006$ & $5 / 4 / 2009$ & 110.88 & 43.41 \\
PRGF & $12 / 21 / 2000$ & $12 / 20 / 2003$ & 110.88 & 27.72 \\
EFF & $05 / 20 / 1996$ & $05 / 19 / 2000$ & 135.00 & 87.50 \\
\hline
\end{tabular}

VI. Projected Obligations to Fund ${ }^{1}$ : (SDR million; based on existing use of resources and present holdings of SDRs):

\begin{tabular}{lrrrrr}
\hline & \multicolumn{5}{c}{ Forthcoming } \\
& 2007 & 2008 & 2009 & 2010 & 2011 \\
\hline Principal & 8.19 & 13.88 & 11.79 & 5.54 & 3.92 \\
Charges/Interest & 1.08 & 0.87 & 0.42 & 0.25 & 0.23 \\
Total & 9.26 & 14.75 & 12.22 & 5.80 & 4.15 \\
\hline
\end{tabular}

\footnotetext{
${ }^{1}$ Disbursements made after November 28, 2000 - with the exception of disbursements of emergency assistance and loans from the Poverty Reduction and Growth Facility - are expected to be repaid on the expectations schedule. Countries may request the IMF Executive Board to make repayments according to the obligations schedule if their external payments position is not strong enough to meet the repayment expectations without undue hardship or risk. Please note: Repayments under the Supplemental Reserve Facility are scheduled to be repaid on the expectations schedule.
} 


\section{Safeguards Assessments:}

Under the Fund's safeguards assessment policy, the National Bank of Moldova (NBM) is subject to an assessment with respect to the PRGF arrangement approved on May 5, 2006, and its subsequent augmentation requested in November 2006. The assessment was completed on October 13, 2006 and it concluded that safeguards in place at the NBM appear adequate. However, certain vulnerabilities were identified in the governance and control system, which will be addressed through implementing the safeguards assessment recommendations.

\section{Exchange Arrangement:}

Moldova has accepted the obligations of Article VIII, Sections 2, 3 and 4, of the Fund's Articles of Agreement. Its exchange system remains free of restrictions on payments and transfers for current international transactions.

Moldova's exchange rate regime was reclassified from a de facto peg (to the U.S. dollar) to a managed float in April 2006.

Prior to the introduction of the Moldovan leu on November 29, 1993, the Russian ruble (supplemented by NBM-issued ruble denominated coupons) was the legal tender in Moldova. The government introduced the leu at a conversion rate of one leu equal to 1,000 Moldovan rubles and an exchange rate of one dollar equal to lei 3.85. The leu remained broadly stable around MDL 4.75/\$ until mid-October 1998. It has since depreciated against the dollar and traded at MDL 12.41 $=\$ 1$ on April 28, 2007.

Foreign exchange was initially traded on the Chişinău Interbank Foreign Currency Exchange (CIFCE). Operations of the CIFCE started at the beginning of 1993, and daily auctions commenced in early February 1995. An active foreign exchange cash market exists within authorized banks and foreign exchange bureaus. From November 16, 1993 through endOctober 1998, the U.S. dollar exchange rate established in the CIFCE was the official exchange rate quoted by the NBM. Since November 2, 1998, the official rate as announced by the NBM is determined as the weighted average of all daily market transactions. The NBM quotes exchange rates of the leu for other currencies on the basis of the leu-U.S. dollar rate and the cross-rate relationships between the U.S. dollar and the currencies concerned in the international market.

\section{Article IV Consultation:}

The last Article IV consultation was concluded on May 5, 2006 (IMF Country Report Nos. 06/184 and 06/187). 


\section{FSAP Participation:}

Moldova received an FSAP mission in May 2004; the FSSA was presented to the Board at the time of the 2004 Article IV discussions. An FSAP update is tentatively scheduled for September 2007.

\section{Use of Fund Resources:}

On February 4, 1993, Moldova purchased Fund resources totaling SDR 13.5 million, equivalent to 15 percent of quota under the Compensatory and Contingency Financing Facilities (CCFF). This was followed by a first drawing under the Systemic Transformation Facility (STF) of SDR 22.5 million, equivalent to 25 percent of quota, which was approved by the Board on September 16, 1993. On December 17, 1993 the Board approved a purchase of Fund resources under a stand-by arrangement (SBA) in the amount of SDR 51.75 million and the drawing of the second tranche of the STF totaling SDR 22.5 million. On December 19, 1994, Moldova made a second purchase under the CCFF totaling SDR 12.2 million, equivalent to 13.5 percent of quota, in conjunction with the second review of the SBA that expired at end-March 1995. The Board approved a successor SBA for Moldova in an amount totaling SDR 58.50 million (equivalent to 65 percent of quota) on March 22, 1995. Three purchases, totaling SDR 32.4 million (equivalent to 36 percent of quota), were made under the arrangement. An Extended Arrangement amounting to SDR 135 million (equivalent to 150 percent of old quota) was approved by the Executive Board on May 20, 1996. At the time of the third review, the program was extended by one year to May 19, 2000. While the fourth review was completed, the program expired on May 19, 2000 without the completion of the scheduled last review because parliament rejected twice an important program condition. Five purchases totaling SDR 87.5 million (equivalent to 97 percent of old quota) were made under the EFF arrangement. A PRGF arrangement was approved on December 21, 2000 in the amount of SDR 110.88 million (equivalent to 90 percent of quota). SDR 9.24 million was disbursed on December 29, 2000, followed by SDR 9.24 million on February 23, 2001, and SDR 9.24 million on July 26, 2002. The arrangement expired in December 2003 with only one review completed. A new PRGF arrangement was approved on May 5, 2006 in the amount of SDR 80.08 million (equivalent to 65 percent of quota). SDR 11.44 million was disbursed on May 10, 2006. In light of twin external shocks (doubling of the import price of natural gas and the Russia's ban imports of Moldovan wine), the access under the program was augmented to SRD 110.88 million (equivalent of 90 percent of quota) at the time of the first review on December 15, 2006. The second disbursement of SDR 31.97 followed on December 21, 2006.

\section{Resident Representative:}

Mr. Mathisen began his assignment in December 2005.

\section{Resident Advisors:}


An FAD resident advisor, Mr. Agarwal, completed a posting to support the development of the treasury in June 1998. Peripatetic return visits were conducted through 1999.

During 1998, Mr. and Mrs. Faulk were assigned as resident advisors on banking supervision at the NBM. During 1999, they paid various follow- up visits as external experts. In July 1999, an FAD resident tax administration advisor, Mr. Vandenberghe, was extended for a third six month period to support the development of a large taxpayer unit. His contract ended in mid-2000. In January 2007, Mr. Pekonen, a resident advisor on monetary policy implementation and liquidity management took up a short-term assignment at the NBM.

\section{Short-Term Assistance:}

Mr. Antao conducted regular visits to assist the ministry of finance on macroeconomic analysis since mid-1997. From April 1996 to July 1998, Mr. Richards paid regular visits as a regional balance of payments statistics advisor to Moldova, Armenia, and Georgia. Mr. Van Sluys and Dr. Peeraer visited Chişinău on several occasions in 1998/99 to assist in the area of supervision of commercial bank's foreign exchange activities. Mr. Thompson has been advising the NBM on accounting issues since 1999 and visited Moldova regularly during the past three and a half years, including for preparation of the NBM international audits. Mr. Ponomarenko visited Moldova several times in 2002-04 to assist the Department of Statistics and Sociology (DSS) in the area of national accounts. Mr. Astin has been visiting Moldova regularly to help the DSS reform the methodology for the CPI and PPI. 


\section{Technical Assistance:}

The following table summarizes the technical assistance missions provided by the Fund to Moldova since January 2001. ${ }^{2}$

Moldova: Technical Assistance Provided by the Fund, 2001-06

\begin{tabular}{|c|c|c|c|}
\hline Department & Subject/Identified Need & Timing & Counterpart \\
\hline $\begin{array}{l}\text { LEG } \\
\end{array}$ & Free economic zone legislation & Jan./Feb. 2001 & $\mathrm{MoE}$ \\
\hline FAD & Expenditure policy & May 2001 & $\mathrm{MoF}$ \\
\hline LEG & Bankruptcy law & June 2001 & MoE \\
\hline LEG & Banking legislation & June 2001 & NBM \\
\hline MAE & TA assessment/evaluation & October 2001 & NBM \\
\hline MAE & $\begin{array}{l}\text { Payments system; liquidity projection and monetary } \\
\text { policy framework; central bank accounting and internal } \\
\text { audit; and bank supervision. }\end{array}$ & May 2002 & NBM \\
\hline MFD & Payments system & $\begin{array}{l}\text { Missions } 2003 \\
\text { and } 2004\end{array}$ & NBM \\
\hline STA & National accounts & $\begin{array}{l}\text { March 2002, } \\
\text { Dec. 2002, } \\
\text { March 2003, } \\
\text { Apr. } 2004\end{array}$ & DSS \\
\hline STA & Price statistics & $\begin{array}{l}\text { Nov. 2003, Sept 2004, } \\
\text { Nov. 2005, July } 2006\end{array}$ & DSS \\
\hline STA & Money and banking statistics & April 2004 & NBM \\
\hline MFD/LEG & Anti-money laundering & April 2004, Aug. 2006 & $\begin{array}{l}\text { MDA Gov. } \\
\text { NBM }\end{array}$ \\
\hline FAD & Modernization of the LTU and VAT & May 2004 & $\mathrm{MoF}$ \\
\hline MFD & Foreign exchange regulation and banking supervision & December 2004 & NBM \\
\hline STA & Money and banking statistics & May 2005 & NBM, DSS \\
\hline MFD & Internal audit (National Bank) & June 2005 & NBM \\
\hline STA & SDDS & November 2005 & $\begin{array}{l}\text { NBM, DSS, } \\
\text { MOF }\end{array}$ \\
\hline MFD & $\begin{array}{l}\text { Central bank independence, monetary policy, and } \\
\text { monetary operations }\end{array}$ & December 2005 & NBM \\
\hline FAD & Public finance management & $\begin{array}{l}\text { March 2006, } \\
\text { Oct. } 2006\end{array}$ & MOF \\
\hline FAD & Tax administration & July 2006 & MOF \\
\hline
\end{tabular}

\footnotetext{
${ }^{2}$ For technical assistance before 2001, see previous reports.
} 


\begin{tabular}{|c|c|c|c|}
\hline MCM & Central bank recapitalization and normalizing relations & August 2006 & NBM, MOF \\
\hline MCM & $\begin{array}{l}\text { Improving monetary policy operations, creating } \\
\text { preconditions for an inflation targeting framework and } \\
\text { analyzing financial sector stability }\end{array}$ & September 2006 & NBM \\
\hline MCM & Development of the foreign exchange system & September 2006 & NBM \\
\hline AD & Tax Policy and Administration & April 2007 & MOF \\
\hline
\end{tabular}

Note: MOF: Ministry of Finance; NBM: National Bank of Moldova; MoE: Ministry of Economy; DSS: Department of State Statistics 
ANNEX II: MOLDOVA-IMF-WORLD BANK RELATIONS

(As of May 15, 2007)

Country Director: Mr. Paul Bermingham

Telephone: +3804449006671

\section{A. Partnership in Moldova's Development Strategy}

1. Moldova's development agenda is set out in the Economic Growth and Poverty Reduction Strategy Paper (EGPRSP). The EGPRSP was presented to the Boards of the International Development Association (IDA) and the IMF in November 2004. In April 2006, the Moldovan authorities prepared the first Annual Evaluation Report (AER). The Joint Staff Advisory Note (JSAN) provided the government with advice on future implementation of the EGPRSP. The document describes the participatory process underpinning the development of the strategy, provides a diagnostic of poverty, and presents sector programs and policy measures for sustainable economic growth and poverty.

2. The IMF has taken the lead in assisting Moldova in promoting and maintaining macroeconomic stability through prudent monetary and financial policies. In this regard the Fund has encouraged the Moldovan authorities to implement a policy reform agenda that would attract the needed level of concessional foreign financing from bilateral and multilateral creditors and encourage the international community to reschedule Moldova's external debt to enhance debt sustainability. The Fund has supported Moldova's economic reform program since 1993. The last three-year PRGF program was approved on May 5, 2006.

3. The World Bank has taken the lead in the policy dialogue on a number of structural issues relevant to economic growth and poverty reduction, including private sector development, infrastructure, health, education, social protection, and agricultural sector reforms.

4. On October 19, 2006 the Bank Executive Board approved a US\$10.0 million Poverty Reduction Support Credit (PRSC) for Moldova. The credit, the first in a planned annual series, aims to support the Government's efforts to accelerate economic growth and improve the efficiency of its social programs and public administration. It directly supports implementation of Moldova's EGPRSP, as well as the EU-Moldova Action Plan. The PRSC will support selected areas of the Government reform agenda with the objective of: (i) improving the investment climate; (ii) improving the efficiency and management of public resources; and (iii) strengthening pension and social assistance systems. It is expected that the implementation of the reform program supported by the PRSC will contribute to improved business environment and investment climate, leading to an increase in the quality of growth and poverty reduction, particularly in the rural areas. A more professional civil service combined with increased transparency and accountability of public finances with improved public expenditure management and strategic allocation of resources is also an important part of the reform program supported by the Credit. Given the recent increases in the price of imported energy, the PRSC also supports the Government's efforts in 
strengthening existing social safety nets for vulnerable groups by completing the 1999 pension reform and improving the targeting of social assistance programs.

5. On December 12, 2006, the World Bank together with the European Commission hosted a Consultative Group Meeting for Moldova. The meeting assessed progress in the implementation of the country's reform program and to provided indications of additional external financing. Moldova's development partners expressed their support for the authorities' strategy and indicated financial support totaling more than US\$ 1.2 billion (almost 1 billion $€$ ) over the next three years, of which $25 \%$ is in budgetary and balance of payment support.

6. Recent analytical work of the Bank includes a Public Expenditure Management Review, an Investment Climate Assessment, a Country Procurement Assessment Review, a Country Financial Accountability Assessment, a Trade Diagnostic Study, the second Poverty Assessment, an Education Policy Note, a Health Policy Note, an Agricultural Note, a Country Economic Memorandum and a Public Expenditure Review. Bank is also providing technical assistance to the government in the areas of poverty monitoring, public administration and civil service reform and governance, and social protection.

7. In a number of areas - social sectors, environment, infrastructure - the Bank takes the lead in the dialogue. The Bank also is leading the areas of support for the private sector development and agricultural sector reform and Bank analysis serves as input into the Fund program. In other areas - trade and customs reform, financial sector policies, public sector management - both institutions work together. Finally, in areas like fiscal, monetary, and exchange rate policies, and tax administration, the IMF takes the lead.

\section{B. IMF-World Bank Collaboration in Specific Areas}

\section{Areas in which the World Bank leads}

8. Areas in which the Bank leads the policy dialogue are social sectors, infrastructure, and environment. In the social sphere, the Bank has carried out Poverty Assessments to monitor and evaluate progress in alleviating poverty. A second poverty assessment has recently been completed. A Social Protection Management Project supports the implementation of comprehensive reform of the public pension system and the introduction of a regulatory framework for private pension funds. The project also supports the implementation of the new organizational structure for social insurance. The Bank has also supported two Social Investment Fund (SIF) Projects to assist in the building of local community and municipal capacity and skills through the rehabilitation of social infrastructure. The SIF also focuses on improving the delivery of social services, creating short-term employment and opportunities in rural areas by financing small-scale public works and supporting micro-business development.

9. In health, the ongoing Health Investment Fund Project is focused on improving the health status of the Moldovan population and increasing the quality and efficiency of public 
health services by improving access to essential services by the poor. The Moldova AIDS Control Project aims at improving Moldova's health status and assists in achieving the health-related Millennium Development Goals by reducing mortality, morbidity, and transmission of HIV/AIDS. An additional operation to help Moldova combat Avian Influenza is under implementation. The Moldova Health Services and Social Assistance Project was approved by the Board in FY07.

10. In education, the Quality Education in Rural Areas Project builds upon recently closed General Education Project and supports the Government's education program to enhance the quality of teaching and learning, increase access and equity, improve the efficiency in public spending for education, and strengthen education planning and monitoring.

11. With regards to infrastructure development, the Bank is concentrating on upgrading basic utility services impacting the population at large and the poor in particular. The Energy II Project has two main components: (i) power system infrastructure-investments to upgrade and rehabilitate metering, dispatch and communications, selected priority rehabilitation of the electricity of the electricity transmission network, and institutional development and regulation of the electricity market; and (ii) heating supply and consumption - investments in improving heating supply and energy efficiency in selected public buildings. A Pilot Water Supply and Sanitation Project is aimed at enhancing the welfare of the population living in some of the poorest rural areas and medium-sized towns and cities by improving the quality, efficiency, and sustainability of water supply and sanitation services by rehabilitating and improving operations of selected water and sewage systems to increase service quality and efficiency, improving the financial viability and commercial practices of the participating utilities, and involving the private sector in the sector. The Moldova Road Sector Program Support Project was approved by the Board in FY07.

12. The Bank has supported a number of environmental projects. The Agriculture Pollution Control Project focuses on significantly increasing the use of environmentally friendly agricultural practices by farmers and agro-industry thereby reducing nutrient discharge from agricultural sources into the Danube River and Black Sea. A number of technical assistance activities are also under implementation. A Biodiversity Strategy Development is assisting the government in implementing Articles 6 and 8 of the Convention on Biological Diversity. The project also supports the formulation and adoption of strategies and actions for the protection and sustainable use of bio-diversity, through a participatory process involving the public and private sectors and local NGOs. The Environmental Infrastructure Project was approved by the Board in FY07. The objectives of the project are to improve the quality of sanitary services in Northern Moldova and reduce the discharge of pollutants, including nutrients into the Nistru River and demonstrate and disseminate costeffective nutrient reduction strategies and technologies for municipal wastewater sources.

13. In the area of rural and agricultural development, the Bank's First Cadastre Project has initiated the first cadastre, implemented a rural land registration system, and helped develop the institutional framework to complete and manage the legal cadastre in urban areas. The project helped establish a system of clear and enforceable ownership rights 
so as to promote the privatization of land the development of real markets in Moldova. The two Rural Investment and Services Projects increase rural incomes and living standards by promoting rural entrepreneurship, agricultural production, economic diversification, and trade in the rural areas. The projects provide public investment support to alleviate key institutional constraints in farmer's group formation, supporting development of marketing institutions and infrastructure for improved access to input and output markets, and developing a rural advisory service. The projects also provide investment and working capital at commercial terms to support a broad range of agribusiness.

14. While the Bank has taken the lead in the areas described above, the IMF has a strong interest in these areas since many of these reforms are critical to achieving macroeconomic stabilization and enhancing growth prospects. Accordingly, there is a high degree of consultation and coordination between the two institutions on these matters.

\section{Areas of shared responsibility}

15. The Bank and Fund are working jointly in a number of areas:

16. Private Sector Development. While substantial progress in improving Moldova's business environment has been made, a challenging reform agenda remains. Both the Bank and the Fund have focused on this agenda in their assistance programs, including SAC III and PRGF arrangement and the surveillance exercise. The Bank has undertaken a number of studies, including the Investment Climate Assessment, the Business Environment and Enterprise Performance Survey (together with EBRD), a Diagnostic Review of the Environment for Foreign Investment, and a number of Costs of Doing Business Surveys, to gauge ongoing developments in this area and determine the most important impediments to private sector development. The Bank's Private Sector Development II (PSD II) Project strengthens the competitiveness of private and public enterprises in Moldova through the hands-on training of local managers abroad (human capital investment) and creation of a Competitiveness Center (market information and benchmarking). The Competitiveness Enhancement Project builds upon successes of the PSD II credit by providing support for the business environment improvement and modernization of the standardization and metrology system. The IFC has promoted financial sector development by extending credit lines to a number of private banks for on-lending to private sector. The Fund has focused on policies needed to maintain a stable macroeconomic environment and remove obstacles to growth, thus encouraging private sector activities. A number of technical assistance missions have been undertaken to improve banking legislation and banking supervision, including the joint Financial Sector Assessment Program.

17. In the area of Public Sector Management, the Bank's Public Economic Management Review, Country Procurement Assessment Review and Country Financial Accountability Assessment examine fiscal adjustment and reform efforts in the social sectors and suggest policy measures to strengthen public expenditure management and actions needed to build capacity for increased government effectiveness. The Country Economic Memorandum entitled Moldova: Opportunities for accelerated growth contains recommendations for 
improving the macroeconomic environment, enhancing the opportunities presented by the large inflow of remittances, creating a more dynamic and diversified private sector. The Bank's project Trade and Transportation Facilitation in Southeast Europe (part of the Stability Pact initiative), aims to strengthen and modernize the Customs Administration and other border control agencies with the objective reducing non-tariff costs to trade and transport and preventing smuggling and corruption at border crossings. The Public Finance Management Project will help the Moldovan Authorities to develop a budget planning and execution system by institutionalizing the medium-term expenditure planning and modernizing budget classification; will assist in upgrading the internal control and audit system in the central government bodies. The Fund has also provided technical assistance to improve tax and customs administration, government financial statistics, and public expenditure policies.

\section{Areas in which the IMF leads}

18. The Fund takes the lead in the formulation and execution of fiscal and monetary policies, external policies, and issues involving economic and financial statistics. In the budgetary area, the Fund leads the dialogue on fiscal matters, taking the lead on tax policy and reforms.

19. In these areas the Bank takes into account the policy recommendations of the IMF and ensures that its own policy advice is consistent.

\section{The World Bank Group Strategy}

20. The main objective of the Country Assistance Strategy (CAS) for Moldova for the period of FY04-FY08 is to support the implementation of the government's EGPRSP and capitalize on the gains of previous assistance programs. Board discussion of the CAS took place in November 2004.

21. A CAS Progress Report was presented for the Executive Board's review on October 19, 2006. It assessed the implementation of the Strategy and discussed adjustments to the program for the remaining two years of the current CAS period. It was proposed to put in place an annual PRSC program of approximately US\$10 million per operation, starting in FY07. The balance of the available IDA allocation would be made available for investment operations drawn from roads, health and social protection, and agriculture and rural development. 
ANNEX III: MOLDOVA-STATISTICAL ISSUES

1. Economic and financial data provided to the Fund are generally adequate for surveillance and program monitoring. In July 2005, a data ROSC Data mission found that while the quality of macroeconomic statistics has improved significantly in many areas in the past few years, accuracy and reliability need to be enhanced regarding compilation of fiscal and national accounts statistics.

2. Moldova began participation in the GDDS in February 2003, and became the $63^{\text {rd }}$ subscriber to SDDS on May 2, 2006.

3. National accounts statistics are prepared according to the 1993 SNA methodology. Estimates do not include the Transnistria region for which data have not been collected since 1991. GDP is estimated from the production and the expenditure sides, annually and quarterly. The data are prepared in current and constant (previous-year) prices, and annual data are revised - in two stages - as updated information becomes available. However, the quarterly data remain unadjusted, thus limiting their consistency with the revised annual data. Overall, the national accounts statistics need improvement in the following areas: (i) the accuracy of the data sources (quarterly reporting of all basic statistics on a discrete basis, industrial production index and price indices following international standards); (ii) benchmarking the annual and the quarterly data; and (iii) estimating the consumption of fixed capital in line with international standards.

4. Price statistics still have several shortcomings. Price collection for the CPI and PPI is limited geographically and new observations are not imputed. The weights of the CPI basket, underestimate the share of durable goods. The PPI is not a transaction price index, and covers a small population of industrial establishments. The most recent STA price statistics mission (mid-2006) found that the main methodological issue of concern for the CPI was the biased treatment of seasonal products. The index captures only the downward part of seasonal fluctuations and misses the price "increases" at the beginning of the season. As for the current PPI compilation, international standards for data collection, validation, and calculation are not followed, mostly due to lack of adequate resources. However, there are plans to implement a few improvements during the rebasing and selection of new respondent companies. Statistics Norway is expected to provide financial aid to hire short-term staff for the visits to new sample establishments.

5. The industrial production index is compiled from data in constant prices adjusted to price changes by establishments themselves.

6. Labor market statistics remain inadequate for analyzing macroeconomic developments: (i) unemployment data following ILO methodology are not available; and (ii) wage data do not include fringe benefits, which form an important part of workers' compensation.

7. Fiscal statistics. A 1999 GFS technical assistance mission assisted the authorities in resolving data issues in the classification of accounts. A Report On the Observance of 
Standards and Codes Data Module prepared in February 2006 found that, from January 2005, in accordance with the 2005 Budget Law, No. 373-XV, 2004, fiscal data, broadly consistent with the GFSM 1986, are produced for the National Public Budget, i.e., the consolidated general government, state budget (all components), local government budgets (all components), State Social Insurance Fund budget, and the Health Insurance Fund budget. The fiscal data cover all economic flows of the general government (except for quasi-fiscal activity of the energy sector) and the stock of general government debt, in accordance with GFSM 1986. The Transnistria region is not included in the compilation of GFS. GFS for the consolidated general government are reported to the IMF in accordance with the classifications of the GFSM 2001 for inclusion in the Government Finance Statistics Yearbook.. In addition, World Bank technical assistance was provided to improve the budgetary reports of local authorities as well as the Social Fund.

8. Balance of payments. The compilation methodology follows the fifth edition of the Balance of Payments Manual. Remaining problems are related to: (i) under-reporting of imports and exports; (ii) collection of data on services; (iii) measurement of private capital inflows, including direct investment data; and (iv) treatment of international transactions of the Transnistria region. Moldova disseminates quarterly international investment position statistics.

9. Monetary and financial statistics (MFS) have improved following a series of technical assistance missions from the Fund. A MFS mission in April 2004 found that while monetary data were broadly in line with international statistical standards and generally of good quality, shortcomings remained in the compilation of some monetary statistics aggregates. A follow-up mission in May 2005 assisted the authorities in implementing the standardized report forms (SRFs), and initiated work toward establishing an Integrated Monetary Database (IMD) for joint use by EUR and STA. The authorities report data in the SRFs to STA on a regular basis. MFS based on the SRFs were published in the December 2006 issue of the quarterly IFS Supplement on Monetary and Financial Statistics. In response to the authorities request, STA plans to field a TA mission in April 2008 to assist in work to expand the coverage of monetary statistics to include other (non depository) financial corporations.. 


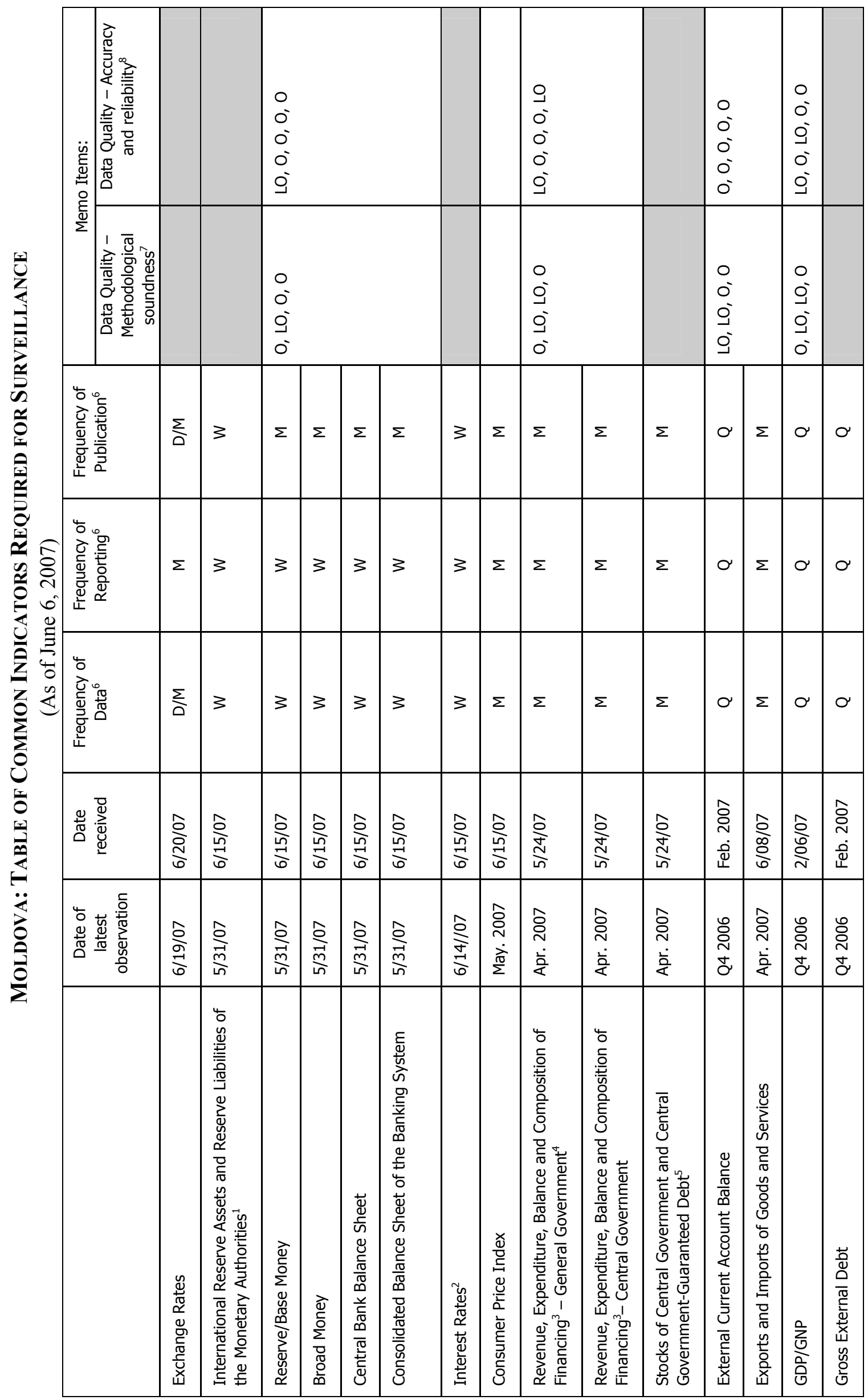




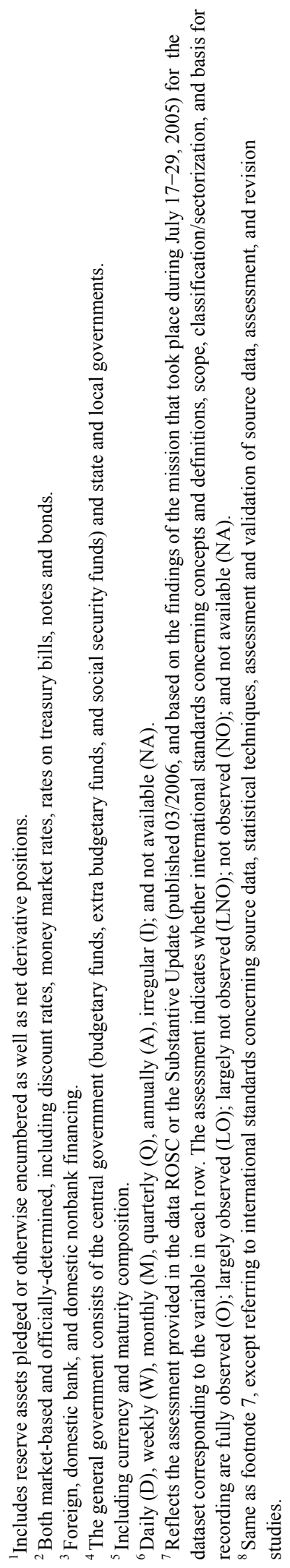





\section{Statement by the IMF Staff Representative July 13, 2007}

1. This statement summarizes developments in Moldova since the issuance of the staff report. The additional information does not change the thrust of the staff appraisal.

2. Recent economic data have been favorable and broadly in line with projections. Real GDP growth in the first quarter of 2007 surprised on the upside at 7.3 percent, led by construction and services. However, dry weather conditions have damaged the grain crop during the second quarter, suggesting that the full year projection of 5 percent remains appropriate. Twelve-month inflation continued to decline to 10.4 percent in June. Monetary targets under the program were observed at end-June, and the NBM's stepped-up sterilization efforts have kept real interest rates on short-term paper in positive territory. Fiscal performance also appears to be broadly on track, with preliminary data showing a cash deficit for the general government of 0.1 percent of annual GDP during the first half, well under the program ceiling of 0.5 percent of GDP. Nevertheless, delays by the municipality of Chişinău in clearing arrears for district heating subsidies mean that expenditure arrears of about 0.3 percent of GDP remained at end-June, implying a continued breach of the indicative ceiling on expenditure arrears. Staff is discussing options for clearing these arrears with the authorities.

3. Preliminary balance of payments data for the first quarter of 2007 show a larger current account deficit than previously expected $(\$ 175$ million, against a projected annual figure of $\$ 354$ million). Compared with the first quarter of 2006, exports increased by 12 percent, although wine exports to Russia remain effectively blocked. Imports grew by 40 percent, reflecting robust inflows of capital and consumer goods, fuelled by higher FDI and strong remittances. The authorities also reported a large positive value for errors and omissions, suggesting that, as in the past, the preliminary quarterly balance of payments numbers could be revised significantly. Specifically, finalized data may well show a smaller current account deficit, as some remittance inflows are reclassified from errors and omissions to the current account.

4. The authorities are amending the AML law, seeking to address concerns raised by the capital amnesty. Key recommendations of recent LEG TA have been incorporated in the draft legislation, which was submitted to parliament on July 2, 2007. Final passage of the law is expected by end-July. Further, the authorities have sought to address AML concerns by consulting with LEG - as well as EU and US experts - in drafting regulations to implement the amnesty. 



\section{IMF Executive Board Completes Second Review of Moldova's PRGF Arrangement and Approves US\$33.1 Million Disbursement}

The Executive Board of the International Monetary Fund (IMF) today completed the second review of Moldova's performance under the three-year Poverty Reduction and Growth Facility (PRGF) arrangement. The completion of the review enables the release of an amount equivalent to SDR 21.7 million (about US\$33.1 million), which will bring the total disbursements to Moldova under the PRGF arrangement to SDR 65.1 million (about US\$99.2 million). The PRGF arrangement was approved on May 5, 2006 (see Press release No. 06/91).

The Executive Board also granted a waiver for the non-observance of the end-December 2006 performance criterion related to tariffs for district utilities.

Following the conclusion of the Executive Board discussion, Mr. Murilo Portugal, Deputy Managing Director and Acting Chairman, stated:

"Moldova's economic performance has been strong, and its implementation of the PRGFsupported program has been encouraging. The authorities' adjustment efforts have lessened the impact of last year's external shocks and helped maintain macroeconomic stability. Growth is accelerating, international reserves are increasing, and inflation - although still high by regional standards - is declining steadily.

"Now that the worst effects of last year's external shocks have passed, the challenge will be to maintain macroeconomic stability and persevere with structural reforms aimed at reducing poverty and bolstering growth by improving the business climate. Accelerating the development of a well-targeted social assistance system should also be a high priority, given possible further increases in energy prices.

"While the fiscal position has been sound, the authorities need to ensure that higher public sector wages, coupled with reduced corporate income taxes and a tax amnesty, will not 
crowd out public investment and limit room for poverty-reducing spending. Rationalizing public sector employment, limiting growth in non-essential spending, and accelerating tax administration reform will be crucial.

"Monetary policy will need to remain tight to bring inflation securely into single digits. Continued exchange rate flexibility, combined with the strengthened capital position of the National Bank of Moldova, will help achieve this goal.

"The authorities are advancing their agenda to improve the business climate and promote financial sector development. In this regard, making the recently established consolidated supervisory body for non-bank financial institutions operational will be crucial. To mitigate reputational risks to the financial sector stemming from the liberalization of rules governing the legalization of capital, it will also be important to ensure that Moldova's anti-money laundering framework is fully compatible with international standards," Mr. Portugal said. 


\section{Statement by Age Bakker, Executive Director for Republic of Moldova and Vladimir Munteanu, Advisor to Executive Director \\ July 13, 2007}

On behalf of the Moldovan authorities, we wish to express appreciation to the staff for the constructive discussions during the review mission, and the resulting well-written and candid report, which reflects the country's recent economic developments and the challenges ahead. Despite the persistent effects of the external shocks encountered in 2006, the authorities have passed, with the support from the international community, the most difficult period of adjustment. This allows them now to focus on achieving the key goals and priorities of the Fund-supported PRGF program.

In light of the satisfactory performance under the program, reflected by the implementation of all quantitative performance criteria and most of the structural conditionality, the authorities request the completion of the second review, and a waiver for non-observance of the structural performance criterion. They also reiterate their commitment to the program's objectives.

\section{Recent economic developments}

The key development since the completion of the last review is the improvement in external outlook and the higher growth perspective. The authorities' decisive adjustment measures, in part unpopular with the general public, in combination with donor support, and an improving external environment helped to weather the effects of the shocks. Despite a doubling in the price of natural gas, albeit somewhat lower than initial expectations, and thanks to a gradual diversification of export markets, and buoyant remittances ${ }^{1}$, real GDP grew at 4 percent in 2006, exceeding staff's more conservative projections. GDP growth is expected to further increase to 5 percent in 2007, and will be sustained at this level over the medium term.

The headline inflation continued to decline and reached 10.4 percent (y-o-y) in June, from 14.1 percent at the end of 2006, while the cumulative inflation during the first six months of 2007 was 3.9 percent compared to 7.4 percent during the same period of last year. The NBM's disinflation efforts have been supported by a prudent fiscal policy. The tight fiscal stance in combination with sound debt management also helped to maintain the improvement in debt sustainability, following last year's Paris Club agreement to reschedule Moldova's bilateral debt.

Some progress has been achieved in restoring economic relations with the Russian Federation, and the authorities hope that a resumption of wine exports will follow the recent decision to allow other agricultural products on the Russian market. At the same time, encouraging progress has been made in diversifying exports. The preliminary data for January-May 2007 indicate that the EU, including Romania and Bulgaria, became the main export market for Moldovan goods. Thus, exports to the EU market accounted for

\footnotetext{
${ }^{1}$ Remittances increased by 29 percent in 2006, reaching 33 percent of GDP.
} 
50.9 percent of total exports for the first five months of the year, while the share of exports to the CIS market decreased from 46.2 percent to 37.3 percent during the same period.

\section{Monetary policy}

Robust foreign exchange inflows, and a still high level of dollarization, continued to present challenges to the monetary authorities. Against this background, additional efforts were required on behalf of the National Bank of Moldova (NBM) to rein in inflation. The NBM's stepped-up sterilization operations pushed real interest rates into positive territory, and supported by appreciation of the leu, led to a decline in y-o-y inflation by about 4 percentage points in the first half of 2007. As noted in the staff report, this indicates that the authorities' end-year goal of single-digit inflation is attainable. Despite of this positive development, the authorities understand that interest rates may need to remain relatively high until disinflation is entrenched, and stand ready to further tighten the monetary policy stance if needed.

To achieve its monetary objective - price stability - the NBM will apply indirect monetary policy instruments, which will be facilitated by the strengthened capital position of the NBM, and by the transfer of the remaining deposits of the health and social funds to the single treasury account in the National Bank. The NBM will continue to maintain a floating exchange rate and will intervene on the foreign exchange market mainly to smooth out volatility in the exchange rate. The exchange rate flexibility will enhance the economy's ability to absorb external shocks. At the same time, the authorities expect that by the end of the year international reserves will increase to a level equivalent to about three months of prospective imports.

The NBM is considering the possibility of moving to an inflation targeting (IT) framework at an appropriate point in the future. In this context, it is taking a number of steps to improve the monetary policy framework, and thus to lay the groundwork for IT. When carrying out sterilization of excessive liquidity, the NBM will announce either a maximum interest rate, or the volume of intended sales. To improve coordination between the NBM and the Ministry of Finance and strengthen liquidity management capacity, a joint NBM-MoF liquidity managing committee was created. Furthermore, beginning with the 2008, the law on the budget will no longer oblige the NBM to roll over treasury bills, instead, this issue will be decided at the joint NBM-MoF liquidity committee. In order to ensure that the NBM will have the tools to conduct monetary policy, by September 30, 2007, the Ministry of Finance and the NBM will adopt a plan for securitizing the stock of government debt owed to the NBM by end-March 2008.

\section{Fiscal policy}

Fiscal policy remains prudent, despite growing pressure to increase expenditures on badly needed infrastructure investments and on current expenditures, in an environment of rising poverty and an exodus of population. Fiscal performance continued to be strong with overall tax revenues reaching 33.8 percent of GDP in 2006 due to growing VAT receipts on imports, improved tax administration and compliance. The same tendency persisted in the first half of 2007. The state budget revenues overperformed against the target by 3.8 percent. Acknowledging the importance of fiscal policy in supporting the overall macroeconomic stability, the authorities remain committed to a prudent fiscal stance. This has been clearly 
reflected in the authorities' decision to approve a supplementary budget, which allows allocation of excess revenues collected only through April, although it is expected that the strong revenues will persist for the rest of the year.

To finance the increase in public sector wages, which are insufficient to attract and retain qualified employees, many of whom have opted instead to work abroad resulting in a tight labor market and increasing capacity constraints, the size of the general government wage bill will be set at about 10 percent of GDP. In the same vein, the government intends to begin a medium-term program of public employment rationalization. As a first step in this direction, the Parliament has already approved a reduction in staff of the National Army and institutions under the Ministry of Defense by 600 people. Furthermore, the government will prepare by end-September 2007, a multi-year concept paper, the goal of which is to reduce public employment while attracting and retaining the best performing and most promising staff.

To ease the tax burden and promote investment, the authorities have announced a major reform of corporate income taxation, an amnesty on tax arrears, and a liberalization of the rules governing the legalization of capital. In this context, the corporate income tax rates will be set to zero, except when dividends are paid and non-business expenses are incurred. The authorities believe that in Moldova's environment a total tax amnesty will be effective. Thus, starting with a "clean sheet" will help to reduce corruption and allow to focus all existing resources on improving the tax administration, rather than wasting them on managing the largely uncollectable historic arrears. To this effect, they intend to adopt by end-September an action plan aimed at improving the tax arrears management by enhancing the tax arrears accounting system, introducing prompt and unconditional measures to ensure forced collection of arrears, and by passing legislation for shortening and streamlining the procedure for writing off uncollectable tax arrears. Furthermore, the legislation to consolidate all domestic tax assessing and collecting activities in one agency will be adopted. The authorities are fully aware that this reform entails some risks, including to budget revenue. Therefore, they are ready, if necessary, to take compensating measures to ensure that the fiscal and macroeconomic targets in the program will be achieved.

The main purpose of the capital legalization is to reduce the shadow economy by encouraging both physical and legal persons, exclusively residents of the Republic of Moldova, excluding Transnistria, to declare their assets at market value, specifically the assets acquired during the economic decline in the 90s at very low prices. No cash is allowed to be legalized by legal persons. At the same time, the authorities recognize that the capital legalization could pose some risk for the anti-money laundering regime. To address these risks, they had extensive consultations with the main development partners, including the US, European Commission, MONEYVAL, and the Fund, and intend to accelerate the adoption of amendments to the AML legislation prepared with the latter's assistance, while also adopting implementing regulations aimed at ensuring the AML framework remains robust.

\section{Financial sector}

The financial sector remains generally sound, and has proved resilient to the difficulties associated with the external shocks. The capital adequacy and liquidity ratios, as well as profitability of the banks remain robust. The authorities welcome the arrival of 
several foreign banks in Moldova, which should translate into improved competition and deeper financial intermediation. The authorities remain committed to privatize Banca de Economii (BEM) to a strategic banking investor, which will further foster deepening in the financial market. To this end, they have solicited the assistance of the International Financial Corporation in preparing to bring BEM to market shortly after completion of the evaluation.

To facilitate the development of the non-bank financial market the parliament has passed the legislation to establish the National Commission for the Financial Markets (NCFM), which will begin operations by August 31, 2007. The NCFM will be financially and operationally independent, and, once it reaches its full operational capacity, but not later than September 30, 2008, it will fully acquire the right to issue and revoke licenses for all types of supervised non-banking financial activity. In the meantime, it will have the full right to suspend the licenses of market participants in violation of prudential norms. Moreover, in order to assess the progress in improving prudential regulation, transparency in ownership of the banking sector, and supervision of the non-bank financial sector, the authorities have requested an FSAP update, which is scheduled to take place in early September.

\section{Structural reforms}

On the structural front, the authorities undertook measures aimed at enhancing the economy's resilience to external shocks, developing the financial sector, and limiting government involvement in the economy. To this effect, the parliament approved legislation that ensures the passing on to final consumers the increase in energy prices, the legislation aimed at developing the non-bank financial market, and the law on denationalization and management of public property. Also, the second stage of the regulatory reform is carried out.

To promote trade and investments, the government is implementing its strategy for attracting investment and promoting exports during 2006-15. In this regard, the key priorities are: (i) obtaining autonomous trade preferences from the EU; (ii) conducting a detailed study of the economic implications for Moldova stemming from Romania's accession to the EU; (iii) creating accredited laboratories for testing and ensuring quality of a number of Moldovan products in compliance with the European standards; and (iv) establishing simplified methods of accounting for small and medium-sized enterprises.

The government has also prepared a concept paper on public-private partnerships (PPP), which will offer a wide spectrum of instruments and mechanisms for cooperation and interaction between the public and private sectors to increase infrastructure investment. The concept paper envisages that contingent liabilities that could appear in this process will be transparently reported to the financial market as well as to the parliament.

Moving forward, the authorities' main goals are to reduce poverty and improve the social assistance system ${ }^{2}$, ensure macroeconomic stability, and sustain economic growth by improving public sector efficiency and the business environment. Also, attracting FDIs,

\footnotetext{
2 The authorities intend to develop the methodology for targeted social assistance system by September 30 , 2007, and introduce it throughout Moldova by September 30, 2008.
} 
which remain very low in contrast to other comparable countries in the region despite recent progress made by Moldova, is on top of the authorities' agenda. These objectives are incorporated in the Economic Growth and Poverty Reduction Strategy Paper (EGPRSP), and in the EU-Republic of Moldova Action Plan. Beyond the EGPRSP, which expires at the end of the year, the government's objectives will be outlined in the National Development Plan for 2008-11 (NDP). The NDP will also integrate the key external commitments of the Republic of Moldova into one basic strategy and will provide a single system for monitoring and evaluation. 